ANALYSIS \& PDE

Volume $3 \quad$ No. $4 \quad 2010$

ALEXANDRE FREIRE

MEAN CURVATURE MOTION OF GRAPHS

WITH CONSTANT CONTACT ANGLE AT A FREE BOUNDARY 


\title{
MEAN CURVATURE MOTION OF GRAPHS WITH CONSTANT CONTACT ANGLE AT A FREE BOUNDARY
}

\author{
ALEXANDRE FREIRE
}

\begin{abstract}
We consider the motion by mean curvature of an $n$-dimensional graph over a time-dependent domain in $\mathbb{R}^{n}$ intersecting $\mathbb{R}^{n}$ at a constant angle. In the general case, we prove local existence for the corresponding quasilinear parabolic equation with a free boundary and derive a continuation criterion based on the second fundamental form. If the initial graph is concave, we show this is preserved and that the solution exists only for finite time. This corresponds to a symmetric version of mean curvature motion of a network of hypersurfaces with triple junctions with constant contact angle at the junctions.
\end{abstract}

\section{Time-dependent graphs with a contact angle condition}

We consider a moving hypersurface $\Sigma_{t}$ in $\mathbb{R}^{n+1}$ with normal velocity equal to its mean curvature. We assume $\Sigma_{t}$ to be a graph over a time-dependent open set $D(t) \subset \mathbb{R}^{n}$, not necessarily bounded or connected. The (properly embedded) intersection $(n-1)$-submanifold

$$
\Gamma(t)=\Sigma_{t} \cap \mathbb{R}^{n}=\partial D(t)
$$

is a moving boundary. Along $\Gamma(t)$ we impose a constant-angle condition

$$
\left\langle N, e_{n+1}\right\rangle_{\mid \Gamma(t)}=\beta,
$$

where $0<\beta<1$ is a constant and $N$ is the upward unit normal of $\Sigma_{t}$. Mean curvature motion $(\mathrm{mcm})$ is defined by the law

$$
V_{N}=H,
$$

where $V_{N}=\langle V, N\rangle$, with $V=\partial_{t} F$ the velocity vector in a given parametrization $F(t)$ of $\Sigma_{t}(V$ depends on the parametrization, while $V_{N}$ does not). A particular parametrization yields mean curvature flow:

$$
\partial_{t} F=H N
$$

For graphs, it is natural to consider graph mean curvature motion: If $\Sigma_{t}=\operatorname{graph} w(t)$ for a function $w(t): D(t) \rightarrow \mathbb{R}$, imposing $\left\langle\partial_{t} F, N\right\rangle=H$ with $F(y, t)=[y, w(y, t)]$ for $y \in D(t)$, we find

$$
w_{t}=\sqrt{1+|D w|^{2}} H
$$

MSC2000: 35K55, 53C44.

Keywords: mean curvature flow, triple junctions, free boundaries. 
(and the velocity is vertical, $\partial_{t} F=w_{t} e_{n+1}$ ). With the contact angle condition, we obtain a free boundary problem for a quasilinear PDE

$$
\begin{cases}w_{t}=g^{i j}(D w) w_{i j} & \text { in } \quad D(t), \\ w=0, \quad \beta \sqrt{1+|D w|^{2}}=1 & \text { on } \quad \partial D(t),\end{cases}
$$

where $g^{i j}(D w)=\delta^{i j}-w_{i} w_{j} /\left(1+|D w|^{2}\right)$ is the inverse metric matrix.

Remark. It is easy to see that the constant-angle boundary condition is incompatible with mean curvature flow parametrized over a fixed domain $D_{0}$ : on $\partial D_{0}$ we would have $\left\langle F, e_{n+1}\right\rangle=0$, leading to $\left\langle\partial_{t} F, e_{n+1}\right\rangle=$ 0 , which is incompatible with $\partial_{t} F=H N$ and $\left\langle N, e_{n+1}\right\rangle=\beta$. If we parametrize over a time-dependent domain, mean curvature flow leads to a normal velocity for the moving boundary that is difficult to control; hence we chose to analyze the geometry of the motion in terms of the graph mcm parametrization.

To establish short-time existence (in parabolic Hölder spaces) we will work with a third parametrization of the motion, defined over a fixed domain:

$$
F(t): D_{0} \rightarrow \mathbb{R}^{n+1}, \quad F(x, t)=[\varphi(x, t), u(x, t)] \in \mathbb{R}^{n} \times \mathbb{R},
$$

where $\varphi(t): D_{0} \rightarrow D(t)$ is a diffeomorphism and $F$ is a solution of the parabolic system

$$
F_{t}=g^{i j}(D F) F_{i j}
$$

where $g_{i j}=\left\langle F_{i}, F_{j}\right\rangle$ is the induced metric on $\Sigma_{t}$ and $g^{i j}$ is the inverse metric matrix.

In the first part of the paper (Sections 3 to 8 ) we prove the following short-time existence theorem (on $\left.Q:=D_{0} \times[0, T]\right)$, where by boundary-orthogonal we mean that certain orthogonality conditions at the boundary, specified in Section 3, are satisfied.

Theorem 1.1. Let $\Sigma_{0} \subset \mathbb{R}^{n+1}$ be a $C^{3+\bar{\alpha}}$ graph over $D_{0} \subset \mathbb{R}^{n}$ satisfying the contact and angle conditions at $\partial D_{0}$. There exist $T>0$ depending only on $\Sigma_{0}$, a parametrization $F_{0}=\left[\varphi_{0}, u_{0}\right] \in C^{2+\alpha}\left(D_{0}\right)$ of $\Sigma_{0}$ (where $\alpha=\bar{\alpha}^{2}$ and $\varphi_{0}$ is a boundary-orthogonal diffeomorphism of $D_{0}$ ), and a unique solution $F \in C^{2+\alpha, 1+\alpha / 2}\left(Q^{T} ; \mathbb{R}^{n+1}\right)$ of the system

$$
\begin{cases}\partial_{t} F-g^{i j}(D F) \partial_{i} \partial_{j} F=0, & F=[\varphi, u] \in \mathbb{R}^{n} \times \mathbb{R} \\ u_{\mid \partial D_{0}}=0, & N^{n+1}(D F)_{\mid \partial D_{0}}=\beta,\end{cases}
$$

with initial data $F_{0}$, where $\varphi(t): D_{0} \rightarrow D(t) \subset \mathbb{R}^{n}$ is a boundary-orthogonal diffeomorphism as well.

The system and boundary conditions are discussed in more detail in Section 3. Sections 4, 5, and 6 deal with compatibility at $t=0$, linearization and the verification that the boundary conditions satisfy complementarity. In particular, adjusting the initial diffeomorphism $\varphi_{0}$ to ensure compatibility (Section 4) leads to the loss of differentiability seen in Theorem 1.1. The required estimates in Hölder spaces for the linearized system are described in Section 7 and the proof is concluded (by a fixed-point argument) in Section 8. While the general scheme is standard, details are included since we are dealing with a free boundary problem with somewhat nonstandard boundary conditions. Free boundary-type problems for mean curvature motion of graphs have apparently not been considered previously.

We describe the evolution equations in the rotationally symmetric case in Section 9 (including a stationary example for the exterior problem) and the extension to the case of a graph motion $\Sigma_{t}$ intersecting fixed support hypersurfaces orthogonally in Section 10. 
The original motivation for this work was to establish (by classical parabolic PDE methods) existenceuniqueness for mean curvature motion of networks of surfaces meeting along triple junctions with constant-angle conditions. One can use a motion $\Sigma_{t}$ of graphs with constant contact angle to produce examples of triple junction motion: three hypersurfaces moving by mean curvature meeting along an $(n-1)$ dimensional submanifold $\Sigma(t)$ so that the three normals make constant angles (say, 120 degrees) along $\Gamma(t)$. We simply reflect on $\mathbb{R}^{n}$, so the hypersurfaces are $\Sigma_{t}, \overline{\text { Sigma }} a_{t}$, and $\mathbb{R}^{n}-\bar{D}(t)$. If $\Sigma_{t}=$ graph $w(t)$ with $w>0$, the system is embedded in $\mathbb{R}^{n+1}$. This is mean curvature motion of a "symmetric triple junction of graphs".

Short-time existence holds for general triple junctions of graphs moving by mean curvature with constant 120-degree angles at the junction, provided a compatibility condition holds along the junction (see Section 16). Since the free-boundary problem is easier to understand in the symmetric case, we decided to do this first. In addition, in the present case it is possible to go further towards a geometric global existence result. In the second part of the paper (Sections 11-15), motivated by recent work on lens-type curve networks [Schnürer et al. 2007], we consider continuation criteria and the preservation of concavity. Since we chose to develop these results for graph motion with a free boundary, although the general lines of proof (via maximum principles) have precedents, the details of the arguments are new. For example, Section 12 contains an extension of the maximum principle for symmetric tensors with Neumann-type boundary conditions given in [Stahl 1996], which in our setting allows one to show preservation of weak concavity in general. Section 14 includes a continuation criterion for the flow. The results obtained in Sections 11-15 are summarized in the following theorem, where $h$ denotes the second fundamental form, pulled back to a symmetric 2-tensor on $D(t)$.

Theorem 1.2. If $\Sigma_{0}$ is weakly concave $(h \leq 0$ at $t=0)$, this property is preserved by the evolution. Let $T_{\max }$ be the maximal existence time for the evolution. If the mean curvature of $\Sigma_{0}$ is strictly negative $\left(\sup _{\Sigma_{0}} H=H_{0}<0\right.$ ), then $T_{\max }$ is finite. Assuming $T_{\max }<\infty$, we have

$$
\limsup _{t \rightarrow T_{\max }}\left[\sup _{\Gamma_{t}}\left(|h|_{g}+\left|\nabla^{\tan } h^{\tan }\right|_{g}\right)\right]=\infty
$$

(if $n=2$, in the concave case). If there is no gradient blowup at $T_{\max }$, the hypersurface contracts to a compact convex subset of $\mathbb{R}^{n}$ as $t \rightarrow T_{\max }$.

Remark. We have not yet proved that the diameter tends to zero as $t \rightarrow T_{\max }$, though this seems likely based on the experience with curves [Schnürer et al. 2007], in the absence of gradient blowup. It is an interesting question (even in the concave case, for $n=2$ ) whether gradient blowup can really occur, that is, whether $\sup _{\Gamma_{t}}\left|\nabla^{\tan } h^{\tan }\right|_{g}$ can diverge as $t \rightarrow T_{\max }$, while $|h|_{g}$ remains bounded on $\left.\Gamma_{t}\right)$.

\section{Normal velocity of the moving boundary}

The evolution is naturally supplied with initial data $\Sigma_{0}$, a graph meeting $\mathbb{R}^{n+1}$ at the prescribed angle. Since we are interested in classical solutions in the parabolic Hölder space $C^{2+\alpha, 1+\alpha / 2}$, we expect an additional compatibility condition at $t=0$. We discuss this first for graph $\mathrm{mcm} w(y, t)$.

Denote by $\Gamma(t)$ a global parametrization of $\partial D(t)$ (with domain in a fixed manifold, and space variables left implicit). Differentiating in $t$ the contact condition $w(\Gamma(t), t)=0$, we find

$$
w_{t}+\langle D w, \dot{\Gamma}(t)\rangle=0 .
$$


Denote by $n_{t}$ the unit normal vector field to $\Gamma(t)$, chosen so that the directional derivative $d_{n} w>0$. The contact condition also implies the gradient of $w$ is purely normal:

$$
D w_{\mid \partial D(t)}=\left(d_{n_{t}} w\right) n_{t} .
$$

Combining this with the angle condition, and bearing in mind that $d_{n_{t}} w_{\mid \Gamma(t)}>0$, we find

$$
d_{n_{t}} w=\frac{\beta_{0}}{\beta} \text { on } \partial D(t), \quad \beta_{0}:=\sqrt{1-\beta^{2}} .
$$

(In fact, this is a more convenient form of the angle boundary condition for $w$, since it is linear.) Thus, on $\partial D(t)$,

$$
\frac{1}{\beta} H=\sqrt{1+\left(d_{n_{t}} w\right)^{2}} H=\frac{\partial w}{\partial t}=-\left\langle\dot{\Gamma}(t), n_{t}\right\rangle d_{n_{t}} w=-\dot{\Gamma}_{n}(t) \frac{\beta_{0}}{\beta},
$$

and we find the normal velocity of the moving boundary, independent of the parametrization of $\Gamma_{t}$ :

$$
\dot{\Gamma}_{n}=-\frac{1}{\beta_{0}} H_{\mid \Gamma(t)} .
$$

In particular, this must hold at $t=0$. Note that we don't get a compatibility condition in the usual sense (of a constraint on the 2-jet of the initial data), but instead an equation of motion for the moving boundary. Later, in the fixed-domain formulation, we will have to deal with a real compatibility condition.

Remark. For more general (nonsymmetric, nonflat) triple junctions with 120-degree angles, the condition

$$
H^{1}+H^{2}=H^{3} \quad \text { on } \Gamma(t)
$$

must hold at the junction (for graphs, oriented by the upward normal); this gives a geometric constraint on the initial data, for classical evolution in $C^{2+\alpha, 1+\alpha / 2}$. This automatically holds in the symmetric case $\left(w^{2}=-w^{1}, w^{3} \equiv 0\right)$, since $H^{3}=0$ and $H^{I}=\operatorname{tr}_{g^{I}} d^{2} w^{I}$ for $I=1,2$.

\section{Choice of gauge}

It is traditional in moving boundary problems to parametrize the time-dependent domain $D(t)$ of the unknown $w(y, t)$ by a time-dependent diffeomorphism:

$$
y=\varphi(x, t), \quad \varphi(t): D_{0} \rightarrow D(t),
$$

and then derive the equation satisfied by the coordinate-changed function from the equation for $w$; see, for example, [Baconneau and Lunardi 2004; Solonnikov 2003]. Motivated by work on curve networks [Mantegazza et al. 2004], we will, instead, consider a general parametrization

$$
F: D_{0} \times[0, T] \rightarrow \mathbb{R}^{n+1}, \quad F(x, t)=[\varphi(x, t), u(x, t)] \in \mathbb{R}^{n} \times \mathbb{R},
$$

and derive an equation for $F$ directly from the definition of mean curvature motion,

$$
\left\langle\partial_{t} F, N\right\rangle=H .
$$

We'll still assume $\varphi(t): D_{0} \rightarrow D(t)$ is a diffeomorphism. 
The first and second fundamental forms are given by

$$
g_{i j}=\left\langle F_{i}, F_{j}\right\rangle, \quad A\left(F_{i}, F_{j}\right)=\left\langle F_{i j}, N\right\rangle,
$$

where we have set $D F=F_{i} e_{i}$ and $D^{2} F\left(e_{i}, e_{j}\right)=F_{i j}$, with $\left(e_{i}\right)$ the standard basis of $\mathbb{R}^{n+1}$. The mean curvature is the trace of $A$ in the induced metric:

$$
H=\left\langle g^{i j}(D F) F_{i j}, N\right\rangle \text {. }
$$

The equation for $F$ is

$$
\left\langle\partial_{t} F-g^{i j}(D F) F_{i j}, N\right\rangle=0 .
$$

There is a natural gauge choice yielding a quasilinear parabolic system

$$
\partial_{t} F-g^{i j}(D F) F_{i j}=0 .
$$

We will sometimes refer to this as the split gauge, since in terms of the components $F=[\varphi, u]$ we have the essentially decoupled system

$$
\left\{\begin{array}{l}
\partial_{t} u-g^{i j}(D \varphi, D u) u_{i j}=0, \\
\partial_{t} \varphi-g^{i j}(D \varphi, D u) \varphi_{i j}=0 .
\end{array}\right.
$$

The splitting is useful in stating the boundary conditions

$$
\begin{cases}u_{\mid \partial D_{0}}=0 & \text { (contact condition) } \\ N^{n+1}(D \varphi, D u)_{\mid \partial D_{0}}=\beta & \text { (angle condition). }\end{cases}
$$

We immediately see a problem: we have two scalar boundary conditions for $n+1$ unknowns, and no moving boundary to help! Our solution to this is to introduce $n-1$ additional orthogonality conditions at the boundary for the parametrization $\varphi(t)$. We impose

$$
\left\langle D_{\tau} \varphi, D_{n} \varphi\right\rangle_{\mid \partial D_{0}}=0 \quad \text { (orthogonality condition), }
$$

for any $\tau \in T \partial D_{0}$, where $n$ denotes the inward unit normal to $D_{0}$. (We fix a tubular neighborhood $\mathcal{N}$ of $\partial D_{0}$ and extend $n$ to $\mathcal{N}$ so that $d_{n} n=0$ in $\mathcal{N}$.)

Geometrically, the orthogonality boundary condition has a precedent in a method often adopted when dealing with the evolution of hypersurfaces in $\mathbb{R}^{n+1}$ intersecting a fixed $n$-dimensional support surface orthogonally (see [Struwe 1988], for example), where one replaces vanishing inner product of the unit normals - a single scalar condition — by a stronger Neumann-type condition for the parametrization corresponding to $n-1$ scalar conditions. (More details are given in Section 10.)

The system must also be supplied with initial data. We assume given an initial hypersurface $\Sigma_{0}$, the graph of a $C^{3+\bar{\alpha}}$ function $\tilde{u}_{0}(x)$ defined in the $C^{3+\bar{\alpha}}$ domain $D_{0} \subset \mathbb{R}^{n}$. (The reason for this choice of differentiability class will be seen later.) It would seem natural to set $\varphi_{0}=\operatorname{Id}_{D_{0}}$, but this causes problems related to compatibility; see Section 4 . We do require the 1 -jet of $\varphi_{0}$ at the boundary to be that of the identity:

$$
\varphi_{0 \mid \partial D_{0}}=\mathrm{Id}, \quad D \varphi_{0 \mid \partial D}=\mathbb{q} .
$$

(In particular, the orthogonality condition holds at $t=0$.) 
We need a more explicit expression for the unit normal, and for that we use the "vector product"

$$
\begin{aligned}
\tilde{N}(D \varphi, D u) & :=(-1)^{n} \operatorname{det}\left[\begin{array}{ccc}
e_{1} & \cdots & e_{n+1} \\
D F^{1} & \cdots & D F^{n+1}
\end{array}\right]=(-1)^{n} \operatorname{det}\left[\begin{array}{cccc}
e_{1} & \ldots & e_{n} & e_{n+1} \\
D \varphi^{1} & \ldots & D \varphi^{n} & D u
\end{array}\right] \\
& :=\left[J(D \varphi, D u), J_{\varphi}\right] \in \mathbb{R}^{n} \times \mathbb{R},
\end{aligned}
$$

where $D F^{i} \in \mathbb{R}^{n}$ for $i=1, \ldots n+1, J_{\varphi}>0$ is the Jacobian of $\varphi$ and $(-1)^{n}$ is introduced to make sure the last component is positive. $J(D \varphi, D u)$ is an $\mathbb{R}^{n}$-valued multilinear form, linear in the components $u_{i}$ of $D u$, and of weight $n-1$ in the components of $D \varphi$. It is easy to check that $J(\mathbb{1}, D u)=-D u$. The unit normal is

$$
N(D \varphi, D u)=\tilde{N}(D \varphi, D u) /\left(|J(D \varphi, D u)|^{2}+\left(J_{\varphi}\right)^{2}\right)^{1 / 2} .
$$

Thus the angle condition may be stated in the form

$$
\beta\left[|J(D u, D \varphi)|^{2}+\left(J_{\varphi}\right)^{2}\right]_{\mid \partial D_{0}}^{1 / 2}=J_{\varphi \mid \partial D_{0}},
$$

and we lose nothing by squaring it:

$$
B(D \varphi, D u):=\beta^{2}|J(D u, D \varphi)|^{2}-\beta_{0}^{2}\left(J_{\varphi}\right)_{\mid \partial D_{0}}^{2}=0 .
$$

\section{Compatibility and the choice of $\varphi_{0}$}

Assume $D \varphi_{0 \mid \partial D_{0}}=\llbracket$. Differentiating in $t$ the contact condition $u_{\mid \partial D_{0}}=0$ and evaluating at $t=0$, we find

$$
0=g^{i j}\left(\square, D u_{0}\right) u_{0 i j} \equiv g_{0}^{i j} u_{0 i j} \text { on } \partial D_{0} .
$$

To interpret this condition, consider the mean curvature at $t=0$, on $\partial D_{0}$ :

$$
H_{0}=\frac{1}{v_{0}}\left[\left\langle J\left(\mathbb{\square}, D u_{0}\right), g_{0}^{i j} \varphi_{0 i j}\right\rangle+J_{\varphi_{0}} g_{0}^{i j} u_{0 i j}\right],
$$

where

$$
v_{0}=\left[\left|J\left(\mathbb{\square}, D u_{0}\right)\right|^{2}+J_{\varphi_{0}}^{2}\right]_{\mid \partial D_{0}}^{1 / 2}=\left(\left|D u_{0}\right|^{2}+1\right)_{\mid \partial D_{0}}^{1 / 2}=\frac{1}{\beta},
$$

using the equality

$$
J\left(\mathbb{\square}, D u_{0}\right)=-D u_{0}=-\left(D_{n} u_{0}\right) n=-\frac{\beta_{0}}{\beta} n
$$

on $\partial D_{0}$. (Recall that $\beta_{0}:=\sqrt{1-\beta^{2}}$.) Thus the compatibility condition is equivalent to

$$
H_{0 \mid \partial D_{0}}=-\beta_{0} g_{0}^{i j}\left\langle\varphi_{0 i j}, n\right\rangle_{\mid \partial D_{0}} .
$$

This implies we can't choose $\varphi_{0} \equiv \operatorname{Id}$ (on all of $D_{0}$ ), unless $H_{0 \mid \partial D_{0}} \equiv 0$, a constraint not present in the geometric problem (as seen above). ${ }^{1}$ Instead, regarding $H_{0}$ as given (by $\Sigma_{0}$ ), and using

$$
g_{0}^{i j}=\delta_{i j}-\frac{u_{0 i} u_{0 j}}{v_{0}^{2}}=\delta_{i j}-\beta_{0}^{2} n^{i} n^{j},
$$

\footnotetext{
${ }^{1}$ The compatibility condition $H_{0 \mid \partial D_{0}}=0$ does occur for graph mcm with Dirichlet boundary conditions in a mean-convex domain [Huisken 1989].
} 
we find the compatibility constraint

$$
\left\langle\left(\delta_{i j}-\beta_{0}^{2} n^{i} n^{j}\right) \varphi_{0 i j}, n\right\rangle=-\frac{1}{\beta_{0}} H_{0} \text { on } \partial D_{0} .
$$

Given the zero- and first-order constraints on $\varphi_{0}$, this can also be written as

$$
n^{i} n^{j}\left\langle\varphi_{0 i j}, n\right\rangle=-\frac{1}{\beta^{2} \beta_{0}} H_{0} \text { on } \partial D_{0} .
$$

The next lemma, whose proof is given in Appendix A, shows that this can be solved.

Lemma 4.1. Let $D_{0} \subset \mathbb{R}^{n}$ be a uniformly $C^{3+\alpha}$ domain (possibly unbounded), $h \in C^{\alpha}\left(\partial D_{0}\right)(0<\alpha<1)$.

(i) One can find a diffeomorphism $\varphi \in \operatorname{Diff}^{2+\alpha}\left(D_{0}\right)$ satisfying on $\partial D_{0}$

$$
\varphi=\mathrm{Id}, \quad d \varphi=\llbracket, \quad n \cdot d^{2} \varphi(n, n)=h .
$$

(ii) More generally, given a nonvanishing vector field

$$
e \in C^{1+\alpha}\left(\partial D_{0} ; \mathbb{R}^{n}\right)
$$

with $\langle e, u\rangle \neq 0$ on $\partial D_{0}$, one can find $\varphi \in \operatorname{Diff}^{2+\alpha}\left(D_{0}\right)$ satisfying on $\partial D_{0}$

$$
\varphi=\mathrm{Id}, \quad d_{n} \varphi=e, \quad n \cdot d^{2} \varphi(n, n)=h .
$$

If $\partial D_{0}$ has two components, we may even require $\varphi$ to satisfy the conditions in parts (i) and (ii) at the two components with different functions $h$. (This will be needed in Section 10).

As usual, a domain is uniformly $C^{3+\alpha}$ if at each boundary point there are local charts to the upper half-space (of class $C^{3+\alpha}$ ), defined on balls of uniform radius, and with uniform bounds on the $C^{3+\alpha}$ norms of the charts and their inverses.

Remark 4.2. In particular, $\varphi$ satisfies the orthogonality conditions at $\partial D_{0}$.

Remark 4.3. It is at this step in the proof that we have a drop in regularity: for $C^{2+\alpha}$ local solutions, we require $C^{3+\alpha}$ initial data. While this is not unexpected in free-boundary problems (see, for example, [Baconneau and Lunardi 2004]), I don't know a counterexample to the lemma if $D_{0}$ is assumed to be a $C^{2+\alpha}$ domain.

Remark 4.4. In our application of the lemma, we in fact have $h \in C^{1+\alpha}\left(\partial D_{0}\right)$, but this does not imply higher regularity for $\varphi$.

\section{Linearization}

The evolution equation and boundary conditions in split gauge are

where

$$
\left\{\begin{array}{cc}
F_{t}-g^{i j}(D F) F_{i j} & =0 \\
u_{\mid \partial D_{0}} & =0 \\
B(D \varphi, D u)_{\mid \partial D_{0}} & =0 \\
O(D \varphi)_{\mid \partial D_{0}} & =0
\end{array}\right.
$$

$$
\mathcal{O}(D \varphi):=\left\langle D^{T} \varphi, D_{n} \varphi\right\rangle
$$


Here $D^{T} \varphi=D \varphi-\left(d_{n} \varphi\right)\langle\cdot, n\rangle$ is an $\mathbb{R}^{n}$-valued $(n-1)$-form on $\partial D_{0}$. We'll prove short-time existence for this system (with initial data $u_{0}, \varphi_{0}$ ) in $C^{2+\alpha, 1+\alpha / 2}$ by the usual fixed-point argument based on linear parabolic theory. Given $\bar{F}=[\bar{\varphi}, \bar{u}]$ in a suitable ball in this Hölder space with center $F_{0}=\left[\varphi_{0}, u_{0}\right]$, it suffices to consider the pseudolinearization of the system:

$$
F_{t}-g^{i j}\left(D F_{0}\right) F_{i j}=\left[g^{i j}(D \bar{F})-g^{i j}\left(D F_{0}\right)\right] \bar{F}_{i j}=: \mathscr{F}\left(\bar{F}, F_{0}\right)=: \overline{\mathscr{F}} .
$$

A fixed point of the map $\bar{F} \mapsto F$ corresponds to a solution of the quasilinear equation.

For the nonlinear boundary conditions, we need the honest linearization at $F_{0}$. For the angle condition, a computation using the boundary constraints on $u_{0}$ and $\varphi_{0}$ yields

$$
\frac{1}{2} \mathscr{L}_{0} B[D \varphi, D u]=\beta \beta_{0} d_{n} u-\beta_{0}^{2}\left\langle d_{n} \varphi, n\right\rangle .
$$

The corresponding linear boundary condition will be

$$
\beta \beta_{0} d_{n} u-\beta_{0}^{2}\left\langle d_{n} \varphi, n\right\rangle=\mathscr{B}\left(D \bar{F}, D F_{0}\right):=\overline{\mathscr{B}},
$$

where

$$
2 \mathscr{B}\left(D F^{1}, D F^{2}\right):=B\left(D \varphi_{1}, D u_{1}\right)-B\left(D \varphi_{2}, D u_{2}\right)-\mathscr{L}_{0} B\left(D\left(\varphi_{1}-\varphi_{2}\right), D\left(u_{1}-u_{2}\right)\right),
$$

and we used

$$
-\frac{1}{2} \mathscr{L}_{0}\left[D \varphi_{0}, D u_{0}\right]_{\mid \partial D_{0}}=\beta \beta_{0} d_{n} u_{0}-\beta_{0}^{2}\left\langle d_{n} \varphi_{0}, n\right\rangle_{\mid \partial D_{0}}=0 .
$$

Also, $B\left(D \varphi_{0}, D u_{0}\right)_{\mid \partial D_{0}}=0$, so at a fixed point $B(D \varphi, D u)_{\mid \partial D_{0}}=0$.

Linearizing the orthogonality boundary condition, we find that $\mathscr{L}_{0} \mathcal{O}[D \varphi]$ is the $(n-1)$-form on $\partial D_{0}$ given by

$$
\mathscr{L}_{0} \mathcal{O}[D \varphi](v)=\left(\partial_{j} \varphi^{i}+\partial_{i} \varphi^{j}\right) n^{j}\left(\delta_{i k}-n^{k} n^{i}\right) v^{k}
$$

(summing over repeated indices). The corresponding linear boundary condition is

$$
\left\langle d_{n} \varphi, \operatorname{proj}^{T}(\cdot)\right\rangle+\left\langle D^{T} \varphi, n\right\rangle=-\Omega\left(D \bar{\varphi}, D \varphi_{0}\right)=: \bar{\Omega},
$$

where proj ${ }^{T}$ denotes orthogonal projection $\mathbb{R}^{n} \rightarrow T \partial D_{0}$, and

$$
\Omega\left(D \varphi_{1}, D \varphi_{2}\right):=\mathscr{O}\left(D \varphi_{1}\right)-\mathcal{O}\left(D \varphi_{2}\right)-\mathscr{L}_{0} \mathcal{O}\left[D \varphi_{1}-D \varphi_{2}\right],
$$

and we used

$$
\mathscr{L}_{0} \mathcal{O}\left[D \varphi_{0}\right]_{\mid \partial D_{0}}=\left\langle\left(d_{n} \varphi_{0}\right)^{T}, \cdot\right\rangle+\left\langle D^{T} \varphi_{0}, n\right\rangle_{\mid \partial D_{0}}=0 .
$$

\section{Complementarity}

We wish to apply linear existence theory to the system

$$
F_{t}-g^{i j}\left(D F_{0}\right) F_{i j}=\overline{\mathscr{F}},
$$

with boundary conditions at $\partial D_{0}$

$$
\left\{\begin{array}{l}
u=0 \\
\beta \beta_{0} d_{n} u+\beta_{0}^{2}\left\langle d_{n} \varphi, n\right\rangle=\overline{\mathscr{\beta}}, \\
\left\langle d_{n} \varphi, \operatorname{proj}^{T}(\cdot)\right\rangle+\left\langle D^{T} \varphi, n\right\rangle=-\bar{\Omega}
\end{array}\right.
$$


and initial conditions

$$
u_{t=0}=u_{0}, \quad \varphi_{t=0}=\varphi_{0} .
$$

It is easy to see that the initial data satisfy the linearized boundary conditions, and above we constructed $\varphi_{0}$ so as to guarantee $g^{i j}\left(D u_{0}, D \varphi_{0}\right) u_{0 i j \mid \partial D_{0}}=0$. (There is no first-order compatibility condition for $\varphi_{0}$.) Thus the linear system satisfies the required compatibility at $t=0$.

Since the linearized boundary conditions are slightly nonstandard, we must verify they satisfy the Lopatinski-Shapiro complementarity conditions. We fix $x_{0} \in \partial D_{0}$ and introduce adapted coordinates $(\rho, \sigma)$ in a neighborhood $\mathcal{N}_{0} \subset \mathcal{N}$ of $x_{0}$ in $D_{0}$ :

$$
x \in \mathcal{N}_{0} \quad \Longrightarrow \quad x=\Gamma_{0}(\sigma)+\rho n(\sigma), \quad \sigma=\left(\sigma_{a}\right) \in \mathcal{U},
$$

where $u \subset \mathbb{R}^{n-1}$ is open and $\Gamma_{0}: \mathcal{U} \rightarrow \mathbb{R}^{n}$ is a local chart for $\partial D_{0}$ at $x_{0}$. This defines a basis of tangential vector fields in $\Gamma_{0}(U)$, and we may assume that at $x_{0}$, we have $\left\langle\tau_{a}, \tau_{b}\right\rangle=\delta_{a b}$ and $\nabla_{\tau_{a}} \tau_{b}\left(x_{0}\right)=0$ (for the induced connection on $\left.T \partial D_{0}\right)$. Let $U$ and $\psi$ be defined in $\left(-\rho_{1}, 0\right) \times \mathcal{U} \times[0, T]$ by

$$
U(\rho, \sigma, t)=u\left(\Gamma_{0}(\sigma)+\rho n(\sigma), t\right), \quad \psi(\rho, \sigma, t)=\varphi\left(\Gamma_{0}(\sigma)+\rho n(\sigma), t\right) .
$$

In these coordinates, the induced metric is written in block form as

$$
\left[g\left(D F_{0}\right)\right]=\left[\begin{array}{cc}
\left|\psi_{\rho}\right|^{2}+\left(U_{\rho}\right)^{2} & \left\langle\psi_{\rho}, \psi_{a}\right\rangle+U_{\rho} U_{a} \\
\left\langle\psi_{\rho}, \psi_{a}\right\rangle+U_{\rho} U_{a} & \left\langle\psi_{a}, \psi_{b}\right\rangle+U_{a} U_{b}
\end{array}\right]_{\mid t=0}=\left[\begin{array}{cc}
1 / \beta^{2} & 0 \\
0 & \mathbb{q}_{n-1}
\end{array}\right]
$$

at $t=0$ and $x_{0}$.

We have

$$
U_{\rho \rho}=D^{2} u(n, n)
$$

since $d_{n} n=0$, and

$$
U_{a b}=D^{2} u\left(\tau_{a}, \tau_{b}\right)+D u \cdot \nabla_{\tau_{a}} \tau_{b}=D^{2} u\left(\tau_{a}, \tau_{b}\right) \quad \text { at } x_{0} .
$$

We don't need $U_{\rho a}$, since $g_{\rho a}=0$ at $x_{0}$.

Thus

$$
\operatorname{tr}_{g_{0}} D^{2} u\left(x_{0}\right)=\beta^{2} D^{2} u(n, n)+\sum_{a} D^{2} u\left(\tau_{a}, \tau_{a}\right)=\beta^{2} U_{\rho \rho}+\sum_{a} U_{a a}:=\beta^{2} U_{\rho \rho}+\Delta_{\sigma} U,
$$

and, likewise,

$$
\operatorname{tr}_{g_{0}} D^{2} \varphi\left(x_{0}\right)=\beta^{2} \psi_{\rho \rho}+\Delta_{\sigma} \psi .
$$

For the linearized orthogonality operator, note that, at $x_{0}$,

$$
\mathscr{L}_{0} O[D \psi]=\left(\left\langle\psi_{\rho}, \tau_{a}\right\rangle+\left\langle\psi_{a}, n\right\rangle\right) \tau_{a} .
$$

Putting everything together, the linear system to consider at $x_{0}$ is

$$
\left\{\begin{array}{l}
U_{t}-\beta^{2} U_{\rho \rho}-\Delta_{\sigma} U=0 \\
\psi_{t}-\beta^{2} \psi_{\rho \rho}-\Delta_{\sigma} \psi=0
\end{array}\right.
$$


with boundary conditions

$$
\left\{\begin{array}{l}
\left.U\right|_{\rho=0}=0 \\
\beta_{0}\left\langle\psi_{\rho}, n\right\rangle+\left.\beta U_{\rho}\right|_{\rho=0}=b(\sigma, t) \\
\left\langle\psi_{\rho}, \tau_{a}\right\rangle+\left.\left\langle\psi_{a}, n\right\rangle\right|_{\rho=0}=\omega_{a}(\sigma, t), \quad a=1, \ldots n-1 .
\end{array}\right.
$$

Now take the Fourier transform in $\sigma \in \mathbb{R}^{n-1}$ (corresponding to $\xi \in \mathbb{R}^{n-1}$ ), Laplace transform in $t$ (corresponding to $p \in \mathbb{C}$ ) to obtain

$$
\hat{U}(\rho, \xi, p) \in \mathbb{C}, \hat{\psi}(\rho, \xi, p) \in \mathbb{C}^{n} ; \xi \in \mathbb{R}^{n-1}, \quad p \in \mathbb{C}, \quad \rho<0 .
$$

In transformed variables, we obtain the following system of linear ODE in $\rho<0$, for fixed $(\xi, p)$ :

$$
\left\{\begin{array}{l}
\beta^{2} \hat{U}_{\rho \rho}-\left(p+|\xi|^{2}\right) \hat{U}=0 \\
\beta^{2} \hat{\psi}_{\rho \rho}-\left(p+|\xi|^{2}\right) \hat{\psi}=0
\end{array}\right.
$$

Writing the solution in the form

$$
\left[\begin{array}{l}
\hat{U}(\rho) \\
\hat{\psi}(\rho)
\end{array}\right]=e^{i \rho \gamma}\left[\begin{array}{l}
\hat{U}(0) \\
\hat{\psi}(0)
\end{array}\right]
$$

we find the characteristic equation $\beta^{2} \gamma^{2}+p+|\xi|^{2}=0$, and choose the root $\gamma$ so that $i \gamma=(1 / \beta) \sqrt{\Delta}$ (where $\Delta=p+|\xi|^{2}$ and we take the branch of the square root defined by $\left.\operatorname{Re} \sqrt{\Delta}>0\right)$. Here $(p, \xi) \in \mathscr{A}$, where

$$
\mathscr{A}=\left\{(p, \xi) \in \mathbb{C} \times \mathbb{R}^{n-1}:|p|+|\xi|>0, \operatorname{Re} p>-|\xi|^{2}\right\}
$$

Thus the solutions decay as $\rho \rightarrow-\infty$.

Let $W^{+}$be the space of such decaying solutions; it has complex dimension $n-1$. The relevant boundary operator on $\mathcal{W}^{+}$is

$$
\mathbb{B}\left[\begin{array}{c}
\hat{U} \\
\hat{\psi}
\end{array}\right]=\left[\begin{array}{c}
\hat{U} \\
\beta_{0}\left\langle\hat{\psi}_{\rho}, n\right\rangle+\beta \hat{U}_{\rho} \\
\left\langle\hat{\psi}_{\rho}, \tau_{a}\right\rangle+i \xi_{a}\langle\hat{\Psi}, n\rangle
\end{array}\right]_{\mid \rho=0}=\left[\begin{array}{c}
\hat{U}(0) \\
\beta_{0}(i \gamma)\langle\hat{\psi}(0), n\rangle+i \beta \gamma \hat{U}(0) \\
(i \gamma)\left\langle\hat{\psi}(0), \tau_{a}\right\rangle+i \xi_{a}\langle\hat{\psi}(0), n\rangle
\end{array}\right]
$$

(a vector in $\mathbb{C} \times \mathbb{C} \times \mathbb{C}^{n-1}$ ).

The complementarity condition (see [Eidelman and Zhitarashu 1998], for example) is the statement that $\mathbb{B}$ is a linear isomorphism from $\mathcal{W}^{+}$to $\mathbb{C}^{n+1}$. With respect to the basis $\left\{\hat{U}(0),\langle\hat{\psi}(0), n\rangle,\left\langle\hat{\psi}(0), \tau_{a}\right\rangle\right\}$ of $W^{+}$, the matrix of $\mathbb{B}$ is (in block form)

$$
[\mathbb{B}]=\left[\begin{array}{ccc}
1 & 0 & {[0]_{1 \times(n-1)}} \\
-\sqrt{\Delta} & -\left(\beta_{0} / \beta\right) \sqrt{\Delta} & {[0]_{1 \times(n-1)}} \\
{[0]_{(n-1) \times 1}} & {\left[i \xi_{a}\right]_{(n-1) \times 1}} & -(\sqrt{\Delta} / \beta) \rrbracket_{n-1}
\end{array}\right] .
$$

This is triangular with nonzero diagonal entries for every $(p, \xi) \in \mathscr{A}$. Hence $\mathbb{B}$ is an isomorphism.

\section{Estimates in Hölder spaces}

For the fixed-point argument based on the linear system, we need estimates for $\left\|\mathscr{F}_{\alpha},\right\| \mathscr{B}\left\|_{1+\alpha},\right\| \Omega \|_{1+\alpha}$ of two types, namely mapping and contraction estimates. 
More precisely, for $T>0, R>0$ and $Q^{T}=D_{0} \times[0, T]$ consider the open ball

$$
B_{R}^{T}=\left\{F \in C^{2+\alpha, 1+\alpha / 2}\left(Q^{T}, \mathbb{R}^{n+1}\right):\left\|F-F_{0}\right\|_{2+\alpha}<R,\left.F\right|_{t=0}=F_{0}\right\} .
$$

$\left(F_{0}=\left[\varphi_{0}, u_{0}\right]\right.$ is defined from the initial surface $\Sigma_{0}$, via Lemma 4.1.) Solving the linear system with right-hand side defined by $\bar{F} \in B_{R}^{T}$ defines a map $\mathbb{F}: \bar{F} \mapsto F$, and we need to verify that, for suitable choices of $T$ and $R, \mathbb{F}$ maps into $B_{R}^{T}$ and is a contraction.

The argument that follows is standard, and the experienced reader may want to skip to the statement of local existence in Theorem 8.1. On the other hand, the result is not covered by any general theorem proved in detail in a reference known to the author, and some readers may find it useful to have all the details included. Another reason is that, although the "right-hand sides" are clearly quadratic, without explicit expressions one might run into trouble with compositions - which cause problems in Hölder spaces - or when appealing to Taylor-remainder arguments if the domain is not convex.

The estimates required to document that $\mathbb{F}$ maps into $B_{R}^{T}$ are of the form

$$
\left\|\mathscr{F}\left(\bar{F}, F_{0}\right)\right\|_{\alpha}+\left\|\mathscr{\Re}\left(D \bar{F}, D F_{0}\right)\right\|_{1+\alpha}+\left\|\Omega\left(D \bar{\varphi}, D \varphi_{0}\right)\right\|_{1+\alpha} \rightarrow 0 \quad \text { as } T \rightarrow 0_{+},
$$

and the contraction estimates are of the form

$$
\left\|\mathscr{F}\left(F^{1}, F^{0}\right)-\mathscr{F}\left(F^{2}, F^{0}\right)\right\|_{\alpha}+\left\|\mathscr{B}\left(D F^{1}, D F^{2}\right)\right\|_{1+\alpha}+\left\|\Omega\left(D \varphi^{1}, D \varphi^{2}\right)\right\|_{1+\alpha} \leq \mu(T)\left\|F^{1}-F^{2}\right\|_{2+\alpha},
$$

where $\mu(T) \rightarrow 0$ as $T \rightarrow 0_{+}$.

Notation. The $(\alpha, \alpha / 2)$ norms are taken on $Q^{T}$, the $(1+\alpha,(1+\alpha) / 2)$ norms on $\left.\partial D_{0} \times[0, T]\right)$. Double bars without an index refer to the $(2+\alpha, 1+\alpha / 2)$ norm, single bars to supremum norms over $Q^{T}$, and parabolic norms are indexed by their spatial regularity ( $\alpha$ for $(\alpha, \alpha / 2)$, etc.) In general, we use brackets for Hölder-type difference quotients.

We deal with the estimates for the forcing term $\mathscr{F}$ first. Consider the map

$$
\mathscr{G}: \operatorname{Imm}\left(\mathbb{R}^{n}, \mathbb{R}^{n+1}\right) \rightarrow \mathrm{GL}_{n}
$$

that associates to the linear immersion $A$ the inverse matrix of $\left(\left\langle A_{i}, A_{j}\right\rangle\right)_{i=1}^{n}$, inner products of the rows of $A$. $\mathscr{G}$ is smooth, in particular locally Lipschitz in the space $\mathscr{W}$ of linear immersions. Hence, if $F^{1}, F^{2}$ are maps $Q^{T} \rightarrow \mathbb{R}^{n+1}$ such that $D F^{i} \in C^{\alpha, \alpha / 2}\left(Q^{T}\right)$ and $D F^{i}(z) \in K$ for all $z \in Q^{T}$, where $K \subset \mathcal{W}$ is a fixed compact set, we have the bound

$$
\left\|\mathscr{G}\left(D F^{1}\right)-\mathscr{G}\left(D F^{2}\right)\right\|_{\alpha} \leq c_{K}\left\|D\left(F^{1}-F^{2}\right)\right\|_{\alpha} .
$$

In fact our maps $F^{i}$ are in $C^{2+\alpha, 1+\alpha / 2}$, so $D F^{i} \in C^{1+\alpha,(1+\alpha) / 2}$. From this higher regularity we obtain the decay as $T \rightarrow 0_{+}$. Assuming $\left.F^{1}\right|_{t=0}=\left.F^{2}\right|_{t=0}$, we have

$$
\left|D\left(F^{1}-F^{2}\right)\right| \leq\left[D\left(F^{1}-F^{2}\right)\right]_{t}^{(1+\alpha) / 2} T^{(1+\alpha) / 2} .
$$

Now recall the elementary fact that if $D \subset \mathbb{R}^{n}$ is a uniformly $C^{1}$ domain (not necessarily convex or bounded) and $f \in C^{1}(D)$ with $\alpha \in(0,1)$, we have for the $\alpha$-Hölder difference quotient $|f|^{\alpha}$ the estimate $[f]^{\alpha} \leq C_{D}\|f\|_{C^{1}}$. (Here "uniformly $C^{1}$ " means that $D$ can be covered by countably many balls of a fixed radius, which are domains of $C^{1}$ manifold-with-boundary local charts for $D$, with uniform $C^{1}$ bounds for 
the charts and their inverses. The constant $C_{D}$ depends on those bounds.) Applying this to $D F$, where $F=F^{1}-F^{2}$ vanishes identically at $t=0$, and assuming $T<1$, we obtain

$$
[D F]_{x}^{a} \leq c\left(|D F|+\left|D^{2} F\right|\right) \leq c\left([D F]_{t}^{(1+\alpha) / 2} T^{(1+\alpha) / 2}+\left[D^{2} F\right]_{t}^{\alpha / 2} T^{\alpha / 2}\right) \leq c\|F\| T^{\alpha / 2},
$$

(where $c$ depends on $D_{0}$ ) and similarly for the Hölder difference quotient in $t$ :

$$
[D F]_{t}^{\alpha / 2} \leq[D F]_{t}^{1+\alpha / 2} T^{1 / 2} \leq\|F\| T^{1 / 2},
$$

so we have

$$
\left\|D\left(F^{1}-F^{2}\right)\right\|_{\alpha} \leq c\left\|F^{1}-F^{2}\right\| T^{\alpha / 2} .
$$

We conclude, under the assumption $F^{1}=F^{2}$ at $t=0$

$$
\left\|\mathscr{G}\left(D F^{1}\right)-\mathscr{G}\left(D F^{2}\right)\right\|_{\alpha} \leq c_{K}\left\|F^{1}-F^{2}\right\| T^{\alpha / 2} .
$$

In particular, applying this to $\bar{F}$ and $F_{0}$, we find

$$
\left\|\left(\varphi(D \bar{F})-\varphi\left(D F_{0}\right)\right) D^{2} \bar{F}\right\|_{\alpha} \leq c_{K}\left\|\bar{F}-F_{0}\right\| T^{\alpha / 2}\|\bar{F}\|,
$$

and for $F^{1}$ and $F^{2}$ coinciding at $t=0$

$$
\|\left(\left(\mathscr{G}\left(D F^{1}\right)-\mathscr{G}\left(D F^{2}\right)\right) D^{2} F^{1}\left\|_{\alpha} \leq c_{K}\right\| F^{1}-F^{2}\left\|T^{\alpha / 2}\right\| F^{1} \|,\right.
$$

as well as

$$
\left\|\left(\varphi\left(D F^{2}\right)-\varphi\left(D F_{0}\right)\right)\left(D^{2} F^{1}-D^{2} F^{2}\right)\right\|_{\alpha} \leq c_{K}\left\|F^{2}-F_{0}\right\| T^{\alpha / 2}\left\|F^{1}-F^{2}\right\|,
$$

so we have the mapping and contraction estimates for $\mathscr{F}\left(\bar{F}, F_{0}\right)$ and $\mathscr{F}\left(F^{1}, F_{0}\right)-\mathscr{F}\left(F^{2}, F_{0}\right)$.

Lemma 7.1. Assume $\bar{F}, F_{0}, F^{1}, F^{2}$ are in $C^{2+\alpha, 1+\alpha / 2}\left(Q^{T} ; \mathbb{R}^{n+1}\right)$ and have the same initial values, and that $D \bar{F}, D F_{0}, D F^{1}, D F^{2}$ all take values in the compact subset $K$ of $\operatorname{Imm}\left(\mathbb{R}^{n}, \mathbb{R}^{n+1}\right)$. Then

$$
\begin{aligned}
\left\|\mathscr{F}\left(\bar{F}, F_{0}\right)\right\|_{\alpha} & \leq c_{K}\left\|\bar{F}-F_{0}\right\|\|\bar{F}\| T^{\alpha / 2}, \\
\left\|\mathscr{F}\left(F^{1}, F_{0}\right)-\mathscr{F}\left(F^{2}, F_{0}\right)\right\|_{\alpha} & \leq c_{K}\left(\left\|F^{1}\right\|+\left\|F^{2}-F_{0}\right\|\right) T^{\alpha / 2}\left\|F^{1}-F^{2}\right\| .
\end{aligned}
$$

In particular, if $\bar{F} \in B_{R}^{T}$,

$$
\left\|\mathscr{F}\left(\bar{F}, F_{0}\right)\right\|_{\alpha} \leq c_{0} R T^{\alpha / 2}
$$

If $\bar{F}^{1}, \bar{F}^{2} \in B_{R}^{T}$, we have

$$
\left\|\mathscr{F}\left(\bar{F}^{1}, F_{0}\right)-\mathscr{F}\left(\bar{F}^{2}, F_{0}\right)\right\|_{\alpha} \leq c_{0} T^{\alpha / 2}\left\|\bar{F}^{1}-\bar{F}^{2}\right\| .
$$

(The constant $c_{0}$ depends only on the data at $t=0$, and we assume $T<1, R<1$.)

Turning to the orthogonality boundary condition, first observe that

$$
\begin{aligned}
\Omega\left(D \varphi^{1},\right. & \left.D \varphi^{2}\right) \\
& =\left\langle D^{T} \varphi^{1}, d_{n} \varphi^{1}\right\rangle-\left\langle D^{T} \varphi^{2}, d_{n} \varphi^{2}\right\rangle-\mathscr{L}_{0} \mathscr{O}\left[D \varphi^{1}-D \varphi^{2}\right] \\
& =\left\langle D^{T}\left(\varphi^{1}-\varphi^{2}\right), d_{n} \varphi^{1}\right\rangle+\left\langle D^{T} \varphi^{2}, d_{n}\left(\varphi^{1}-\varphi^{2}\right)\right\rangle-\left\langle d_{n}\left(\varphi^{1}-\varphi^{2}\right), D^{T} \varphi_{0}\right\rangle-\left\langle D^{T}\left(\varphi^{1}-\varphi^{2}\right), d_{n} \varphi_{0}\right\rangle \\
& =\left\langle D^{T} \varphi^{1}-D^{T} \varphi^{2}, d_{n} \varphi^{1}-d_{n} \varphi_{0}\right\rangle+\left\langle d_{n} \varphi^{1}-d_{n} \varphi^{2}, D^{T} \varphi^{2}-D^{T} \varphi_{0}\right\rangle,
\end{aligned}
$$


which has quadratic structure. Using a local frame $\left(\tau_{a}\right)_{a=1}^{n-1}$ for $T \partial D_{0}$, we find the components

$$
\Omega_{a}\left(D \varphi^{1}, D \varphi^{2}\right)=\left[\partial_{i}\left(\varphi^{1}-\varphi^{2}\right) \partial_{j}\left(\varphi^{1}-\varphi_{0}\right)+\partial_{j}\left(\varphi^{1}-\varphi^{2}\right) \partial_{i}\left(\varphi^{2}-\varphi_{0}\right)\right] n^{j} \tau_{a}^{i} .
$$

The summation convention is $i, j=1, \ldots, n$, so $\Omega_{a}$ is a sum of terms of the form

$$
b(x) D\left(\varphi^{1}-\varphi^{2}\right) D\left(\varphi^{3}-\varphi^{4}\right),
$$

where $b(x)=n^{j} \tau_{a}^{i}$ and the $\varphi^{I}$ coincide at $t=0$. It is then not hard to show that

$$
\left\|b(x) D\left(\varphi^{1}-\varphi^{2}\right) D\left(\varphi^{3}-\varphi^{4}\right)\right\|_{1+\alpha} \leq c\|b\|_{1+\alpha}\left\|\varphi^{1}-\varphi^{2}\right\|\left\|\varphi^{3}-\varphi^{4}\right\| T^{\alpha},
$$

with $c$ depending on the $C^{1}$ norms of local charts for $D_{0}$. To bound the norm $\left\|n \otimes \tau_{a}\right\|_{1+\alpha}$, note that $|n|\left|\tau_{a}\right| \leq 1,\left|D\left(n \otimes \tau_{a}\right)\right| \leq|D n|+\left|D \tau_{a}\right|$, and $\left[D\left(n \otimes \tau_{a}\right)\right]_{x}^{\alpha} \leq[D n]_{x}^{\alpha}+\left[D \tau_{a}\right]_{x}^{\alpha}$. Since $n=-\left(\beta / \beta_{0}\right) D u_{0}$ on $\partial D_{0}$ and $\partial D_{0}$ is a level set of $u_{0}$, we clearly have

$$
\|D n\|_{\alpha}+\left\|D \tau_{a}\right\|_{\alpha} \leq c\left\|D^{2} u_{0}\right\|_{\alpha} \leq c\left\|u_{0}\right\| .
$$

We summarize the conclusion in the following lemma:

Lemma 7.2. Assume $\bar{\varphi}, \varphi_{0}, \varphi^{1}, \varphi^{2} \in C^{2+\alpha, 1+\alpha / 2}\left(Q^{T} ; \mathbb{R}^{n}\right)$ have the same initial values. Then

$$
\left\|\Omega\left(D \bar{\varphi}, D \varphi_{0}\right)\right\|_{1+\alpha} \leq c_{0}\left\|u_{0}\right\|\left\|\bar{\varphi}-\varphi_{0}\right\|^{2} T^{\alpha}
$$

and

$$
\left\|\boldsymbol{\Omega}\left(D \varphi^{1}, D \varphi^{2}\right)\right\|_{1+\alpha} \leq c_{0}\left\|u_{0}\right\|\left(\left\|\varphi^{1}-\varphi_{0}\right\|+\left\|\varphi^{2}-\varphi_{0}\right\|\right) T^{\alpha}\left\|\varphi^{1}-\varphi^{2}\right\|
$$

with $c_{0}$ depending only on the data at $t=0$. In particular, if $\bar{F}=[\bar{\varphi}, \bar{u}] \in B_{R}^{T}$, we have

$$
\left\|\Omega\left(D \bar{\varphi}, D \varphi_{0}\right)\right\|_{1+\alpha} \leq c_{0} R^{2} T^{\alpha},
$$

and for $\bar{F}^{I}=\left[\bar{\varphi}^{I}, \bar{u}^{I}\right] \in B_{R}^{T}, I=1,2$, we have

$$
\left\|\boldsymbol{\Omega}\left(D \bar{\varphi}^{1}, D \bar{\varphi}^{2}\right)\right\|_{1+\alpha} \leq c_{0} R T^{\alpha}\left\|\bar{\varphi}^{1}-\bar{\varphi}^{2}\right\| .
$$

To explain the estimates for the angle condition, we write the normal vector as a multilinear form on $D F^{i}$

$$
\tilde{N}(D F)=J_{n}(D F):=(-1)^{n} \sum_{i=1}^{n+1}(-1)^{i-1}\left(D F^{1} \wedge \ldots \hat{D F^{i}} \wedge \ldots D F^{n+1}\right) e_{i} \in \mathbb{R}^{n+1}
$$

( $D F^{i}$ omitted in the $i$-th term of the sum), where $D F^{i} \in \mathbb{R}^{n}$ for $i=1, \ldots, n+1$ and we identify the $n$-multivector in $\mathbb{R}^{n}$ with a scalar, using the standard volume form. The angle condition has the form

$$
\beta^{2}|\tilde{N}|^{2}-\left\langle\tilde{N}, e_{n+1}\right\rangle^{2}=0 \text { on } \partial D_{0},
$$

and we set

$$
B(D F):=\beta^{2}\left|J_{n}(D F)\right|^{2}-\left\langle J_{n}(D F), e_{n+1}\right\rangle^{2},
$$

with linearization at $D F_{0}=\left[\square_{n} \mid D u_{0}\right]$

$$
\mathscr{L}_{0} B[D F]=2 \beta^{2}\left\langle J_{n}\left(D F_{0}\right), D J_{n}\left(D F_{0}\right)[D F]\right\rangle-2\left\langle J_{n}\left(D F_{0}\right), e_{n+1}\right\rangle\left\langle D J_{n}\left(D F_{0}\right)[D F], e_{n+1}\right\rangle .
$$


Under the assumption $F^{1}=F^{2}$ at $t=0$, we need an estimate in $C^{1+\alpha,(1+\alpha) / 2}$ for $\mathscr{B}\left(D F^{1}, D F^{2}\right)$

$$
\begin{aligned}
:= & B\left(D F^{1}\right)-B\left(D F^{2}\right)-\mathscr{L}_{0} B\left[D F^{1}-D F^{2}\right] \\
= & \beta^{2}\left(\left|J_{n}\left(D F^{1}\right)\right|^{2}-\left|J_{n}\left(D F^{2}\right)\right|^{2}-2\left\langle J_{n}\left(D F_{0}\right), D J_{n}\left(D F_{0}\right)\left[D F^{1}-D F^{2}\right]\right\rangle\right) \\
& -\left(\left\langle J_{n}\left(D F^{1}\right), e_{n+1}\right\rangle^{2}-\left\langle J_{n}\left(D F^{2}\right), e_{n+1}\right\rangle^{2}-2\left\langle J_{n}\left(D F_{0}\right), e_{n+1}\right\rangle\left\langle D J_{n}\left(D F_{0}\right)\left[D F^{1}-D F^{2}\right], e_{n+1}\right\rangle\right) .
\end{aligned}
$$

It will suffice to estimate the expression in the first parenthesis; the second is analogous.

We need the following algebraic observation: if $T_{0}=\left[\square_{n} \mid D u_{0}\right]$ and $T$ are $n \times(n+1)$ matrices, the expression

$$
\left|J_{n}\left(T_{0}+T\right)\right|^{2}-\left|J_{n}\left(T_{0}\right)\right|^{2}-2\left\langle J_{n}\left(T_{0}\right), D J_{n}\left(T_{0}\right)[T]\right\rangle
$$

is a linear combination (with constant coefficients) of terms of the form

$$
u_{0 i} p_{(2)}(T), \quad u_{0 i} u_{0 j} p_{(2)}(T), \quad p_{(2)}(T),
$$

where the $p_{(2)}(T)$ are polynomials in the entries of $T$ (with constant coefficients) with terms of degree $2 \leq$ deg $\leq 2 n$.

Thus $\mathscr{B}\left(D F^{1}, D F^{2}\right)$ is a linear combination (with constant coefficients) of terms

$$
u_{0 i} p_{(2)}\left(D F^{1}-D F^{2}\right), \quad u_{0 i} u_{0 j} p_{(2)}\left(D F^{1}-D F^{2}\right), \quad p_{(2)}\left(D F^{1}-D F^{2}\right),
$$

with the $p_{(2)}$ as described, and hence it is a linear combination of terms of the form

$$
u_{0 i}\left(F_{k}^{1 j}-F_{k}^{2 j}\right)^{d}, \quad u_{0 i} u_{0 l}\left(F_{k}^{1 j}-F_{k}^{2 j}\right)^{d}, \quad\left(F_{k}^{1 j}-F_{k}^{2 j}\right)^{d}
$$

(where $2 \leq d \leq 2 n, 1 \leq j \leq n+1,1 \leq i, l, k \leq n$ ), which we write symbolically as

$$
\mathscr{B}\left(D F^{1}, D F^{2}\right) \sim \sum_{2 \leq d \leq 2 n} b(x)\left(D F^{1}-D F^{2}\right)^{d},
$$

where $b(x)$ is constant or $u_{0 i}(x)$ or $u_{0 i}(x) u_{0 j}(x)$. For the degree $d$ terms $G^{(d)} \sim b(x)\left(D F^{1}-D F^{2}\right)^{d}$, it is not hard to show the bound

$$
\left\|G^{(d)}\right\|_{1+\alpha} \leq c\|b\|_{1+\alpha}\left\|F^{1}-F^{2}\right\|^{d} T^{\alpha}, \quad 2 \leq d \leq 2 n .
$$

We conclude:

Lemma 7.3. Assume $\bar{F}, F_{0}, F^{1}, F^{2}$ are in $C^{2+\alpha, 1+\alpha / 2}\left(Q^{T} ; \mathbb{R}^{n+1}\right)$ and have the same initial values. Then

$$
\begin{gathered}
\left\|\mathscr{B}\left(D \bar{F}, D F_{0}\right)\right\|_{1+\alpha} \leq c\left(1+\left\|u_{0}\right\|^{2}\right)\left(1+\left\|\bar{F}-F_{0}\right\|^{2 n-2}\right) T^{\alpha}\left\|\bar{F}-F_{0}\right\|^{2} . \\
\left\|\mathscr{B}\left(D F^{1}, D F^{2}\right)\right\|_{1+\alpha} \leq c\left(1+\left\|u_{0}\right\|^{2}\right)\left(1+\left\|F^{1}-F^{2}\right\|^{2 n-2}\right) T^{\alpha}\left\|F^{1}-F^{2}\right\|^{2}
\end{gathered}
$$

with $c$ depending only on $F_{0}$. In particular, if $\bar{F} \in B_{R}^{T}$ then

$$
\left\|\Re\left(D \bar{F}, D F_{0}\right)\right\|_{1+\alpha} \leq c_{0} R^{2} T^{\alpha},
$$

and if $\bar{F}^{1}, \bar{F}^{2} \in B_{R}^{T}$ then

$$
\left\|\Re\left(D \bar{F}^{1}, D \bar{F}^{2}\right)\right\|_{1+\alpha} \leq c_{0} T^{\alpha}\left\|\bar{F}^{1}-\bar{F}^{2}\right\|,
$$

with $c_{0}$ depending only on $F_{0}$. 


\section{Local existence}

Given a $C^{3+\bar{\alpha}}$ graph $\Sigma_{0}$ over a uniformly $C^{3+\bar{\alpha}}$ domain $D_{0} \subset \mathbb{R}^{n}$ (for arbitrary $\bar{\alpha} \in(0,1)$ ) satisfying the contact and angle conditions, let $\varphi_{0} \in \operatorname{Diff}^{2+\bar{\alpha}}$ be the diffeomorphism given by Lemma 4.1 (with the 1-jet of the identity at $\partial D_{0}$ and 2-jet determined by the mean curvature of $\Sigma_{0}$ at $\partial D_{0}$ ). Then find $u_{0} \in C^{2+\alpha}\left(D_{0}\right)$ so that $F_{0}=\left[\varphi_{0}, u_{0}\right] \in C^{2+\alpha}\left(D_{0} ; \mathbb{R}^{n+1}\right)$ parametrizes $\Sigma_{0}$ over $D_{0}\left(\alpha=\bar{\alpha}^{2}<\bar{\alpha}\right)$.

(Precisely, if $\left[z, \tilde{u_{0}}(z)\right]$ parametrizes $\Sigma_{0}$ as a graph, and $\varphi_{0}$ is given by Lemma 4.1, let $u_{0}=\tilde{u_{0}} \circ \varphi_{0}$; so $u_{0} \in C^{2+\alpha}$.)

We obtained in Section 7 all the estimates needed for a fixed-point argument in the set

$$
B_{R}^{T}=\left\{F \in C^{2+\alpha, 1+\alpha / 2}\left(Q^{T}, \mathbb{R}^{n+1}\right):\left\|F-F_{0}\right\|<R,\left.F\right|_{t=0}=F_{0}\right\} .
$$

Choose $R<1$ and $T_{0}<1$ small enough (depending only on $F_{0}$ ) so that, for $F \in B_{R}^{T_{0}}, F(t)=[\varphi(t), u(t)]$ defines an embedding of $D_{0}$, with $\varphi(t)$ a diffeomorphism onto its image $D(t)$. Let $K \subset \operatorname{Imm}\left(\mathbb{R}^{n}, \mathbb{R}^{n+1}\right)$ be a compact set containing $D F(z)$ for all $F \in B_{R}$ and $z \in Q^{T_{0}}$. Now consider $T<T_{0}$.

Given $\bar{F} \in B_{R}^{T}$, solve the linear system (LPDE)/(LBC) with initial data $F_{0}$ to get $F \in C^{2+\alpha, 1+\alpha / 2}\left(Q^{T}\right)$. (This is possible since the complementarity and compatibility conditions hold for the linear system.) This defines a map $\mathbb{F}: \bar{F} \mapsto F$.

From linear parabolic theory (see [Eidelman and Zhitarashu 1998, theorem VI.21], for example), we have

$$
\left\|F-F_{0}\right\| \leq M\left(\left\|\mathscr{F}\left(\bar{F}, F_{0}\right)\right\|_{\alpha}+\left\|\mathscr{B}\left(D \bar{F}, D F_{0}\right)\right\|_{1+\alpha}+\left\|\Omega\left(D \bar{\varphi}, D \varphi_{0}\right)\right\|_{1+\alpha}\right),
$$

where $M>0$ depends on the $C^{\alpha, \alpha / 2}$ norm of the coefficients of the linear system, that is, ultimately on $\left\|F_{0}\right\|$.

From Lemmas 7.1-7.3, it follows that

$$
\left\|F-F_{0}\right\| \leq M c_{0}\left(R T^{\alpha / 2}+R^{2} T^{\alpha}\right)<R
$$

provided $T$ is chosen small enough (depending only on $F_{0}$.) Thus $\mathbb{F}$ maps $B_{R}^{T}$ to itself.

Similarly, if $\mathbb{F}\left(\bar{F}^{i}\right)=F^{i}$ for $i=1,2$, standard estimates for the linear system solved by $F^{1}-F^{2}$ give

$$
\left\|F^{1}-F^{2}\right\| \leq M\left(\left\|\mathscr{F}\left(\bar{F}^{1}, \bar{F}^{2}\right)\right\|_{\alpha}+\left\|\mathscr{P}\left(D \bar{F}^{1}, D \bar{F}^{2}\right)\right\|_{1+\alpha}+\left\|\Omega\left(D \bar{\varphi}^{1}, D \bar{\varphi}^{2}\right)\right\|_{1+\alpha}\right)
$$

Again the estimates in Lemmas 7.1-7.3 imply

$$
\left\|F^{1}-F^{2}\right\| \leq M c_{0}\left(T^{\alpha / 2}+T^{\alpha}\right)\left\|\bar{F}^{1}-\bar{F}^{2}\right\|<\frac{1}{2}\left\|\bar{F}^{1}-\bar{F}^{2}\right\|,
$$

assuming $T$ is small enough (depending only on $F_{0}$ ). This concludes the argument for local existence.

Theorem 8.1. Let $\Sigma_{0} \subset \mathbb{R}^{n+1}$ be a $C^{3+\bar{\alpha}}$ graph over $D_{0} \subset \mathbb{R}^{n}$ satisfying the contact and angle conditions at $\partial D_{0}$. With $\alpha=\bar{\alpha}^{2}$, there exists a parametrization $F_{0}=\left[\varphi_{0}, u_{0}\right] \in C^{2+\alpha}\left(D_{0}\right)$ of $\Sigma_{0}$, a number $T>0$ depending only on $F_{0}$ and a unique solution $F \in C^{2+\alpha, 1+\alpha / 2}\left(Q^{T} ; \mathbb{R}^{n+1}\right)$ of the system

$$
\left\{\begin{array}{l}
\partial_{t} F-g^{i j}(D F) \partial_{i} \partial_{j} F=0, \quad F=[\varphi, u] \\
u_{\mid \partial D_{0}}=0, \quad N^{n+1}(D \varphi, D u)_{\mid \partial D_{0}}=\beta, \quad\left\langle D^{T} \varphi, d_{n} \varphi\right\rangle_{\mid \partial D_{0}}=0
\end{array}\right.
$$


with initial data $F_{0}$. For each $t \in[0, T], F(t)$ is a $C^{2+\alpha}$ embedding parametrizing a surface $\Sigma_{t}$ which satisfies the contact and angle conditions and moves by mean curvature. In addition, $F(t)$ satisfies the orthogonality condition at $\partial D_{0}$.

The hypersurfaces $\Sigma_{t}$ are graphs. For each $t \in[0, T], \varphi(t): D_{0} \rightarrow D(t)$ is a diffeomorphism and $\Sigma_{t}=\operatorname{graph}(w(t))$ for $w(t): D(t) \rightarrow \mathbb{R}$ given by $w(t)=u(t) \circ \varphi^{-1}(t)$. (Since $w(t)$ lies in $C^{2+\alpha^{2}}(D(t))$, it is less regular than $u(t)$ or $\varphi(t)$.) $D(t)$ is a uniformly $C^{2+\alpha}$ domain.

Remark. This theorem does not address the geometric uniqueness of the motion, given $\Sigma_{0}$. It only asserts uniqueness for solutions of the parametrized flow (including the orthogonality boundary condition) in the given regularity class.

\section{Rotational symmetry}

In this section we record the equations for two rotationally symmetric instances of the problem:

(i) $D_{0}$ and $D(t)$ are disks, and $u>0$ (lens case).

(ii) $D_{0}$ and $D(t)$ are complements of disks in $\mathbb{R}^{n}$ (exterior case). For simplicity we restrict to $n=2$.

Let $F(r)=[\varphi(r), u(r)]$ parametrize a hypersurface $\Sigma$, where $\varphi(r)=\phi(r) e_{r}$ is a diffeomorphism onto its image. Here $e_{r}$ and $e_{\theta}$ are orthonormal vectors, outward normal and counterclockwise tangent, respectively, to the circles $r=$ const. The unit upward normal vector and mean curvature are

$$
N=\frac{\left[-u_{r} e_{r}, \phi_{r}\right]}{\sqrt{u_{r}^{2}+\phi_{r}^{2}}} \quad \text { and } \quad H=\frac{1}{\left(\phi_{r}^{2}+u_{r}^{2}\right)^{3 / 2}}\left(\phi_{r} M\left(\phi_{r}, u_{r}\right)\left[D^{2} u\right]-\left\langle u_{r} e_{r}, \vec{M}\left(\phi_{r}, u_{r}\left[D^{2} \varphi\right]\right\rangle\right)\right.
$$

where

$$
\mathcal{M}\left(\phi_{r}, u_{r}\right)\left[D^{2} u\right]=u_{r r}+\left(\phi_{r}^{2}+u_{r}^{2}\right) \frac{u_{r} \phi_{r}}{\phi^{2}}, \quad \vec{M}\left(\phi_{r}, u_{r}\right)\left[D^{2} \varphi\right]=\left[\phi_{r r}+\left(\phi_{r}^{2}+u_{r}^{2}\right)\left(\frac{r \phi_{r}}{\phi^{2}}-\frac{1}{\phi}\right)\right] e_{r} .
$$

Simplifying we get

$$
H=\frac{1}{\left(\phi_{r}^{2}+u_{r}^{2}\right)^{3 / 2}}\left[\phi_{r} u_{r r}-u_{r} \phi_{r r}+\left(\phi_{r}^{2}+u_{r}^{2}\right) \frac{u_{r}}{\phi}\right] .
$$

Now consider the time-dependent case $F(r, t)=\left[\phi(r, t) e_{r}, u(r, t)\right]$. From the expressions above, one finds easily that the equation $\left\langle\partial_{t} F, N\right\rangle=H$ takes the form

$$
\phi_{r}\left(u_{t}-\frac{1}{\phi_{r}^{2}+u_{r}^{2}} \mathcal{M}\left(\phi_{r}, u_{r}\right)\left[D^{2} u\right]\right)=u_{r}\left\langle e_{r}, \varphi_{t}-\frac{1}{\phi_{r}^{2}+u_{r}^{2}} \overrightarrow{\mathcal{M}}\left(\phi_{r}, u_{r}\right)\left[D^{2} \varphi\right]\right\rangle .
$$

In split gauge, we consider the system

$$
\left\{\begin{array}{l}
u_{t}-\frac{1}{\phi_{r}^{2}+u_{r}^{2}} \mathcal{M}\left(\phi_{r}, u_{r}\right)\left[D^{2} u\right]=0, \\
\varphi_{t}-\frac{1}{\phi_{r}^{2}+u_{r}^{2}} \vec{M}\left(\phi_{r}, u_{r}\right)\left[D^{2} \varphi\right]=0 .
\end{array}\right.
$$

Note that $\phi(r, t)=r$ solves the $\phi$ equation, and that in this case the $u$ equation becomes

$$
w_{t}-\frac{w_{r r}}{1+w_{r}^{2}}-\frac{w_{r}}{r}=0
$$


This can be compared with the equation for curve networks,

$$
w_{t}-\frac{w_{x x}}{1+w_{x}^{2}}=0
$$

The boundary conditions are easily stated (we assume $D_{0}$ is the unit disk or its complement). The "contact condition" at $r=1$ is $u=0$. For the "angle condition" at $r=1$, we find

$$
u_{r}^{2}=\frac{\beta_{0}^{2}}{\beta^{2}} \phi_{r}^{2}, \quad \beta_{0}:=\sqrt{1-\beta^{2}} .
$$

Assuming $\phi_{r}>0$ at $r=1$, this resolves as

$$
\begin{array}{lll}
\beta u_{r}+\beta_{0} \phi_{r}=0 & \text { at } r=1 & \text { (lens case), } \\
\beta u_{r}-\beta_{0} \phi_{r}=0 & \text { at } r=1 & \text { (exterior case). }
\end{array}
$$

(For lenses, one also has at $r=0: u_{r}=0$ and $\phi_{r}=1$.) Thus in both cases one can work with linear Dirichlet/Neumann-type boundary conditions.

One reason to consider the exterior case is that, unlike the lens case, it admits stationary solutions. Geometrically one just has to consider one-half of a catenoid truncated at an appropriate height. For example, for 120-degree junctions the equation for stationary solutions

$$
\begin{cases}\frac{u_{r r}}{1+u_{r}^{2}}+\frac{u_{r}}{r}=0 & \text { in }\{r>1\} \\ u_{r \mid r=1}=\sqrt{3}, & u_{\mid r=1}=0 .\end{cases}
$$

admits the explicit solution

$$
u(r)=\frac{\sqrt{3}}{2}\left(\ln \left(2 r+\sqrt{4 r^{2}-3}\right)-\ln 3\right), \quad r>\sqrt{3} / 2 .
$$

Problem. It would be interesting to consider the nonlinear dynamical stability of this solution (even linear stability is yet to be considered). One may even work with bounded domains by introducing a fixed boundary at some $R>1$ intersecting the surface orthogonally (see Section 10).

\section{Fixed supporting hypersurfaces}

Extending the local existence theorem to the case of hypersurfaces intersecting a fixed hypersurface $\mathscr{S}$ orthogonally presents no essential difficulty. The case of vertical support surface leads directly to graph evolution with a standard Neumann condition on a fixed boundary; we consider the complementary case where $\mathscr{S}$ is a graph. Let $\mathscr{S} \subset \mathbb{R}^{n+1}$ be a $C^{4}$ embedded hypersurface (not necessarily connected), the graph over $\mathscr{D} \subset \mathbb{R}^{n}$ of $B \in C^{4}(\mathscr{D})$, oriented by the upward unit normal

$$
v(y):=\frac{1}{v_{B}} \tilde{v}(y), \quad \tilde{v}(y):=[-D B(y), 1] \in \mathbb{R}^{n} \times \mathbb{R}, \quad v_{B}:=\sqrt{1+|D B(y)|^{2}} .
$$

We assume $v$ to be nowhere vertical in $\mathscr{D}(D B \neq 0)$. To state the problem in the graph parametrization, we consider a time-dependent domain $D(t) \subset \mathbb{R}^{n}$ with a boundary consisting of two components $\partial_{1} D(t)$ 
and $\partial_{2} D(t)$, both moving. The hypersurface $\Sigma_{t}$ is the graph of $w(\cdot, t)$ over $D(t)$ solving the parabolic equation

$$
w_{t}-g^{i j}(D w) w_{i j}=0 \quad \text { in } E:=\bigcup_{t \in[0, T]} D(t) \times\{t\} \in \mathbb{R}^{n+1} \times[0, T]
$$

with boundary conditions

$$
w(\cdot, t)_{\mid \partial_{1} D(t)}=0, \quad{\sqrt{1+|D w|^{2}}}_{\mid \partial_{1} D(t)}=1 / \beta
$$

(as before), and on $\partial_{2} D(t)$

$$
w=B, \quad \nabla w \cdot \nabla B=-1 .
$$

(The first-order condition on $\partial_{2} D(t)$ is equivalent to $\langle v, N\rangle=0$.)

Differentiating in $t$ the boundary condition $w=B$ leads easily to an equation for the normal velocity of the interface $\Gamma(t)=\partial_{2} D(t)$ :

$$
\dot{\Gamma}_{n}=\frac{v H}{B_{n}-w_{n}} .
$$

Note that $w_{n}$ at $\partial_{2} D(t)$ can be computed from $B_{n}$, since

$$
-1=\nabla w \cdot \nabla B=w_{n} B_{n}+\left|\nabla^{T} B\right|^{2}
$$

in particular neither $B_{n}$ nor $w_{n}$ can vanish (so both have constant sign on connected components of $\partial_{2} D$ ), and one easily computes: $w_{n}-B_{n}=-v_{B}^{2} / B_{n}$.

Let $\Lambda=\Sigma \cap \mathscr{S}$ be the intersection (n-1)-manifold, the graph of $w$ (or $B$ ) over $\partial_{2} D$. Given the graph parametrizations of $\Sigma$ and $\mathscr{Y}$, say

$$
G(y)=[y, w(y)], \quad \mathbb{B}(y)=[y, B(y)], \quad y \in \partial_{2} D,
$$

and $\tau \in T \partial_{2} D$, we have the tangent vectors

$$
G_{n}:=\left[n, w_{n}\right] \in T \Sigma, \quad G_{B}:=[\nabla B,-1]=-v_{B} v \in T \Sigma, \quad G_{\tau}:=[\tau, \nabla w \cdot \tau] \in T \Lambda,
$$

and the second fundamental forms of $\Sigma$ and $\mathscr{Y}$ (for $e \in \mathbb{R}^{n}$ arbitrary):

$$
A(d G e, d G e)=\frac{1}{v} d^{2} w(e, e), \quad \mathscr{A}(d \mathbb{B} e, d \mathbb{B} e)=\frac{1}{v_{B}} d^{2} B(e, e) .
$$

From the equality $\langle v, N\rangle=0$ at $\partial_{2} D$, it follows easily that (compare [Stahl 1996])

$$
A\left(G_{\tau}, v\right)=-\mathscr{A}\left(G_{\tau}, N\right), \quad \tau \in T \partial D
$$

For the remainder of this section, we concentrate on the boundary conditions at $\partial_{2} D_{0}$. To establish short-time existence, we consider as before the parametrized flow

$$
F_{t}-\operatorname{tr}_{g} d^{2} F=0, \quad g=g(d F), \quad F=[\varphi, u] .
$$

The contact and angle boundary conditions are

$$
u_{\mid \partial_{2} D_{0}}=B \circ \varphi_{\mid \partial_{2} D_{0}}, \quad\langle N, v \circ \varphi\rangle_{\mid \partial_{2} D_{0}}=0 .
$$


Again we have two scalar boundary conditions for $n+1$ components. Here the solution is easier than at the junction. With the notation $F_{n}=d F n=\left[\varphi_{n}, u_{n}\right]$, we replace the angle condition by the "vector Neumann condition"

$$
F_{n} \perp T \mathscr{S} \text { or } F_{n}=-\alpha v_{B} v \text { on } \partial_{2} D_{0},
$$

where $\alpha: \partial_{2} D_{0} \rightarrow \mathbb{R}$, or equivalently (since this leads to $\alpha=-u_{n}$ )

$$
\varphi_{n}=-u_{n}(\nabla B \circ \varphi) \text { on } \partial_{2} D_{0}
$$

Clearly the Neumann condition implies the angle condition $\langle N, v \circ \varphi\rangle=0$, but not conversely. This linear Neumann-type condition can easily be incorporated into the fixed-point existence scheme described earlier.

There is one issue to consider: the zero- and first-order compatibility conditions must hold at $\partial_{2} D_{0}$ at $t=0$. The initial hypersurface $\Sigma_{0}$ uniquely determines $w_{0}$ and $D_{0} \subset \mathbb{R}^{n}$ (satisfying $w_{0}=B$ and $\nabla w_{0} \cdot \nabla B=-1$ on $\left.\partial_{2} D_{0}\right)$, and then once $\varphi_{0} \in \operatorname{Diff}\left(D_{0}\right)$ is fixed, $u_{0}=w_{0} \circ \varphi_{0}$ is also determined. We may assume

$$
\varphi_{0}=i d, \quad \varphi_{0 n}=\nabla B \text { on } \partial_{2} D_{0},
$$

so

$$
u_{0 n}=\nabla w_{0} \cdot \varphi_{0 n}=\nabla w_{0} \cdot \nabla B=-1 \text { on } \partial_{2} D_{0},
$$

and then the Neumann condition $F_{0 n \mid \partial_{2} D_{0}}=-v_{B} v$ holds at $t=0$, on $\partial_{2} D_{0}$.

The first-order compatibility condition is

$$
\operatorname{tr}_{g} d^{2} u_{0}=u_{t}=\nabla B \cdot \varphi_{t}=\nabla B \cdot \operatorname{tr}_{g} d^{2} \varphi_{0} \text { on } \partial D_{0}
$$

or equivalently

$$
\operatorname{tr}_{g}\left\langle\nu, d^{2} F_{0}\right\rangle=0 \text { on } \partial D_{0} .
$$

(This is not a mean curvature condition; the mean curvature of $\Sigma_{0}$ is $H=\operatorname{tr}_{g}\left\langle N, d^{2} F_{0}\right\rangle$.)

From now on we omit the subscript 0 but continue to discuss compatibility at $t=0$. First observe that the Neumann condition leads to a splitting of the induced metric. Given $\tau \in T \partial_{2} D_{0}$, let $F_{\tau}=d F \tau \in T \Lambda$. Then (recalling $u_{n}=-1$ on $\partial_{2} D_{0}$ )

$$
\left\langle F_{\tau}, F_{n}\right\rangle=\left\langle[\tau, d B \tau],\left[\varphi_{n}, u_{n}\right]\right\rangle=\nabla B \cdot \tau-\nabla B \cdot \tau=0
$$

Thus we have

$$
\operatorname{tr}_{g}\left\langle v, d^{2} F\right\rangle=g^{a b}\left\langle v, d^{2} F\left(\tau_{a}, \tau_{b}\right)\right\rangle+g^{n n}\left\langle v, d^{2} F\left(F_{n}, F_{n}\right)\right\rangle,
$$

for a local basis $\left\{T_{a}=d F \tau_{a}\right\}_{a=1}^{n-1}$ of $T \Lambda$ with $g_{a b}=\left\langle T_{a}, T_{b}\right\rangle$ and $g_{n n}=\left|F_{n}\right|^{2}=v_{B}^{2}$.

Differentiating in $n$ the condition $u_{n}=\nabla w \cdot \varphi_{n}$ (assuming, as usual, that $n$ is extended to a tubular neighborhood $\mathcal{N}$ of $\partial_{2} D_{0}$ as a self-parallel vector field) we find

$$
u_{n n}=d^{2} w(n, \nabla B)+\nabla w \cdot d^{2} \varphi(n, n) .
$$


(This is legitimate, since $u=w \circ \varphi$ throughout $\mathcal{N}$.) This is used to compute

$$
\begin{aligned}
\left\langle\nu, d^{2} F(n, n)\right\rangle & =\frac{1}{v_{B}}\left[u_{n n}-\nabla B \cdot d^{2} \varphi(n, n)\right] \\
& =\frac{1}{v_{B}}\left[d^{2} w(n, \nabla B)+(\nabla w-\nabla B) \cdot d^{2} \varphi(n, n)\right] \\
& =-v A\left(G_{n}, v\right)+\frac{1}{v_{B}}\left(w_{n}-B_{n}\right) n \cdot d^{2} \varphi(n, n) .
\end{aligned}
$$

Bearing in mind the expression for $w_{n}-B_{n}$ found earlier, the compatibility condition may be stated in the form

$$
\frac{v_{B}}{B_{n}} n \cdot d^{2} \varphi(n, n)=-v A\left(G_{n}, v\right)+g^{a b}\left\langle d^{2} F\left(\tau_{a}, \tau_{b}\right), v\right\rangle .
$$

We are now in the same situation as in Section 4. Given the 1-jet of $\varphi_{0}$ on $\partial_{2} D_{0}$, we extend $\varphi_{0}$ to a tubular neighborhood $\mathcal{N}$ of $\partial_{2} D_{0}$ (and then to all of $D_{0}$ ) so that $n \cdot d^{2} \varphi(n, n)$ has the value on $\partial_{2} D_{0}$ dictated by the compatibility condition, using Lemma 4.1(ii). We just need to verify that the right-hand side of the expression above depends only on $\Sigma_{0}, \mathscr{Y}$ and the 1 -jet of $\varphi_{0}$ over $\partial_{2} D_{0}$. Clearly only the term $g^{a b}\left\langle v, d^{2} F\left(\tau_{a}, \tau_{b}\right)\right\rangle$ is potentially an issue.

Fix $p \in \partial_{2} D_{0}$ and let $\left\{\tau_{a}\right\}$ be an orthonormal frame for $T \partial_{2} D_{0}$ near $p$, parallel at $p$ for the connection induced on $\partial_{2} D_{0}$ from $\mathbb{R}^{n}$. If $\mathscr{K}$ denotes the second fundamental form of $\partial_{2} D_{0}$ in $\mathbb{R}^{n}$, we have

$$
\tau_{a}\left(\tau_{b}\right)=\mathscr{K}\left(\tau_{a}, \tau_{b}\right) n \text { at } p
$$

on the left-hand-side, $\tau_{b}$ is regarded as a vector-valued function in $\mathbb{R}^{n}$. Still computing at $p$, this implies

$$
\begin{aligned}
d^{2} F\left(\tau_{a}, \tau_{b}\right) & =\tau_{a}\left(d F \tau_{b}\right)-d F\left(\tau_{a}\left(\tau_{b}\right)\right)=\tau_{a}\left(d \mathbb{B} \tau_{b}\right)-\mathscr{K}\left(\tau_{a}, \tau_{b}\right) F_{n} \\
& =d^{2} \mathbb{B}\left(\tau_{a}, \tau_{b}\right)+\mathscr{K}\left(\tau_{a}, \tau_{b}\right) \mathbb{B}_{n}-\mathscr{K}\left(\tau_{a}, \tau_{n}\right) F_{n},
\end{aligned}
$$

where $F_{n}=-v v$ and $\mathbb{B}_{n}=d \mathbb{B} n \in T \mathscr{S}$. Hence

$$
\left\langle v, d^{2} F\left(\tau_{a}, \tau_{b}\right)\right\rangle=\left\langle\nu, d^{2} \mathbb{B}\left(\tau_{a}, \tau_{b}\right)\right\rangle+v \mathscr{K}\left(\tau_{a}, \tau_{b}\right)=\mathscr{A}\left(T_{a}, T_{b}\right)+v \mathscr{K}\left(\tau_{a}, \tau_{b}\right) .
$$

This clearly depends only on $\mathscr{S}$ and on $\Sigma_{0}$. We summarize the discussion in a lemma.

Lemma 10.1. Let $\Sigma_{0}=\operatorname{graph}\left(w_{0}\right)$ be a $C^{3}$ graph over $D_{0} \subset \mathbb{R}^{n}$ (a uniformly $C^{3}$ domain) intersecting a fixed hypersurface $\mathscr{S}=\operatorname{graph}(B)$ over $\partial D_{0}$. Consider the parametrized mean curvature motion with Neumann boundary condition

$$
\begin{gathered}
F \in C^{2,1}\left(D_{0} \times[0, T]\right) \rightarrow \mathbb{R}^{n+1}, \quad F=[\varphi, u], \\
F_{t}-\operatorname{tr}_{g} d^{2} F=0, \quad g=g(d F), \quad u \circ \varphi=B \quad \text { and } \quad F_{n} \perp T \mathscr{S} \text { on } \partial D_{0} .
\end{gathered}
$$

Then $\varphi_{0} \in \operatorname{Diff}\left(D_{0}\right)$ can be chosen so that (with $\left.u_{0}=w_{0} \circ \varphi_{0}\right)$ the initial data $F_{0}=\left[\varphi_{0}, u_{0}\right]$ satisfies the zero- and first-order compatibility conditions at $t=0$ and $\partial D_{0}$ :

$$
\varphi_{0 n}=-u_{0 n}\left(\nabla B \circ \varphi_{0}\right), \quad\left\langle v \circ \varphi_{0}, \operatorname{tr}_{g_{0}} d^{2} F_{0}\right\rangle=0 .
$$

Remark. Differentiating $d w \tau_{a}=d B \tau_{a}$ along $\tau_{b}$, we find

$$
d^{2} w\left(\tau_{a}, \tau_{b}\right)-d^{2} B\left(\tau_{a}, \tau_{b}\right)=\left(w_{n}-B_{n}\right) \mathscr{K}\left(\tau_{a}, \tau_{b}\right)
$$


(reminding us that, although $w \equiv B$ on $\partial D_{0}$, the tangential components of their Hessians do not coincide.) From this follows the expression for $\mathscr{Y}$ in terms of $A$ and $\mathscr{A}$ :

$$
\mathscr{K}\left(\tau_{a}, \tau_{b}\right)=\frac{1}{w_{n}-B_{n}}\left[v A\left(T_{a}, T_{b}\right)-v_{B} \mathscr{A}\left(T_{a}, T_{b}\right)\right] .
$$

It is also easy to express the corresponding traces in terms of the mean curvatures $H^{\Lambda}$ and $\mathscr{H}^{\Lambda}$ of $\Lambda$ in $\Sigma$ and $\mathscr{Y}$ :

$$
H^{\Lambda}=\frac{v}{v_{B}} g^{a b} A\left(T_{a}, T_{b}\right), \quad \mathscr{H}^{\Lambda}=\frac{v_{B}}{v} g^{a b} \mathscr{A}\left(T_{a}, T_{b}\right)
$$

\section{Boundary conditions for the second fundamental form}

To understand the long-term behavior of a graph $\left(\Sigma_{t}\right)$ in $\mathbb{R}^{n+1}$ moving by mean curvature and intersecting $\mathbb{R}^{n}$ at a constant angle, we need to consider the evolution of its second fundamental form. Working in the graph parametrization the boundary conditions are easy to state and linear:

$$
w_{\mid \partial D(t)}=0, \quad d_{n} w_{\mid \partial D(t)}=\frac{\beta_{0}}{\beta},
$$

where $n=n_{t}$ is the inner unit normal to $\partial D(t)$. It is possible to reparametrize the $\Sigma_{t}$ over a different time-dependent domain $\mathscr{D}(t)$, obtaining mean curvature flow

$$
\mathscr{F}_{t}: \mathscr{D}(t) \rightarrow \mathbb{R}^{n+1}, \quad \partial_{t} \mathscr{F}=H N,
$$

with boundary conditions

$$
\mathscr{F}_{\mid \partial \mathscr{D}(t)}^{n+1}=0, \quad N_{\mid \partial \mathscr{D}(t)}^{n+1}=\beta .
$$

For this parametrization the evolution equation for the second fundamental form (and its covariant derivatives of arbitrary order) is well-understood [Huisken 1984]. The disadvantage is that the unit normal $N_{\mid \mathscr{F}_{t}}$ depends nonlinearly on the components of $\mathscr{F}$, and as a result the boundary conditions for the second fundamental form (which are needed for global estimates over spacetime domains) do not admit simple expressions. Therefore we choose to work with graph flow at the cost of having to derive and understand a new set of evolution equations. The equations for $h$ and the mean curvature $H$ are derived in Appendix B. In this section we derive boundary conditions. The development is similar that in [Stahl 1996] for MCF of hypersurfaces intersecting a fixed boundary orthogonally.

It is easy to see that $h$ splits on $\partial D(t)$ : if $\tau \in T \partial D(t)$ is a tangential vector field, and $n=n_{t}$ is the inner unit normal

$$
h(n, \tau)=\frac{1}{v} d^{2} w(n, \tau)=\frac{1}{v}\left(\tau\left(w_{n}\right)-D w \cdot \bar{\nabla}_{\tau} n\right)=0 \text { on } \partial D(t),
$$

since $w_{n} \equiv \beta_{0} / \beta$ on the boundary and $\bar{\nabla}_{\tau} n \in T \partial D(t)$ ( $\bar{\nabla}$ is the euclidean connection). In particular, it follows that $h(D w, \tau)=0$ on $\partial D(t)$.

Remark. Already this simple fact cannot be shown for $a(v, \tau)$, the second fundamental form in the MCF parametrization, regarded as a quadratic form on $\mathscr{D}(t)$. 
Boundary condition for $\boldsymbol{H}$. In Section 2 we derived the equation for the normal velocity of the moving boundary $\Gamma_{t}=\partial D(t)$ :

$$
\dot{\Gamma}_{n}=-\frac{v}{w_{n}} H=-\frac{1}{\beta_{0}} H \text { at } \partial D(t) .
$$

Since $\left\langle N, e_{n+1}\right\rangle(\Gamma(t), t) \equiv \beta$ on $\partial D(t)$ we have

$$
\left\langle\partial_{t} N, e_{n+1}\right\rangle=-\left\langle\partial_{k} N, e_{n+1}\right\rangle \dot{\Gamma}^{k},
$$

where $\partial_{k} N=-g^{i j} h_{i k} G_{j}$ with $e_{n+1}$ component

$$
\left\langle\partial_{k} N, e_{n+1}\right\rangle=-g^{i j} w_{j} h_{i k}=-\frac{1}{v^{2}} h\left(D w, \partial_{k}\right)=-\frac{1}{v^{2}} w_{n} h\left(n, \partial_{k}\right) .
$$

Hence we find, on $\partial D(t)$,

$$
\left\langle\partial_{t} N, e_{n+1}\right\rangle=\frac{w_{n}}{v^{2}} h(n, \dot{\Gamma})=\frac{w_{n}}{v^{2}} \dot{\Gamma}_{n} h(n, n)=-\beta H h_{n n} .
$$

(We set $\left.h_{n n}:=h(n, n)\right)$. Denote by $\nabla^{\Sigma}$ the gradient of $\Sigma_{t}$, in the induced metric $\left(\nabla^{\Sigma} f=g^{i j} f_{i} G_{j}\right)$. Using $\partial_{t} N=-\nabla^{\Sigma} H-H v^{-1} \nabla^{\Sigma} v$, combined with the expressions (valid on $\partial D(t)$ )

$$
\begin{gathered}
\left\langle\nabla^{\Sigma} H, e_{n+1}\right\rangle=g^{i j} H_{i}\left\langle G_{j}, e_{n+1}\right\rangle=g^{i j} H_{i} w_{j}=\frac{1}{v^{2}} w_{i} H_{i}=\frac{w_{n}}{v^{2}} H_{n}=\beta \beta_{0} H_{n}, \\
\left\langle\nabla^{\Sigma} v, e_{n+1}\right\rangle=\frac{v_{n} w_{n}}{v^{2}}=\frac{w_{n}^{2}}{v^{2}} h_{n n}=\beta_{0}^{2} h_{n n},
\end{gathered}
$$

we find on $\partial D(t)$

$$
\left\langle\partial_{t} N, e_{n+1}\right\rangle=-\beta \beta_{0}\left(H_{n}+\beta_{0} H h_{n n}\right) .
$$

Comparing expressions for $\left\langle\partial_{t} N, e_{n+1}\right\rangle$ in (11-1) and (11-2) yields a Neumann-type condition for $H$. We state this as a lemma (including the evolution equation derived in Appendix B). Here $L=L_{g}$ denotes the operator $L[f]=\partial_{t} f-\operatorname{tr}_{g} D^{2} f$ and $\omega=D w / v$, a vector field in $D(t)$.

Lemma 11.1. For the surfaces $\Sigma_{t}$ evolving by graph mean curvature motion with constant contact angle, the mean curvature satisfies

$$
\begin{cases}L[H]=|h|_{g}^{2} H+H h^{2}(\omega, \omega)-H^{2} h(\omega, \omega) & \text { on } D(t), \\ d_{n} H=\left(\beta^{2} / \beta_{0}\right) H h_{n n} & \text { on } \partial D(t) .\end{cases}
$$

Boundary conditions for $\boldsymbol{h}_{i j}$. Fix $p \in \partial D(t)$ and let $\left(\tau_{a}\right)$ be an orthonormal frame for $T_{p} \partial D(t)$ (in the induced metric) satisfying $\nabla_{\tau_{a}}^{\Gamma} \tau_{b}(p)=0$, where $\nabla^{\Gamma}$ is the connection induced on $\Gamma_{t}$ by the euclidean connection $d$, or, equivalently, by $\nabla$, the Levi-Civita connection of the metric $g$ in $D(t)$. We extend the $\tau_{a}$ to a tubular neighborhood of $\Gamma_{t}$ so that $\bar{\nabla}_{n} \tau_{a}=0$. Differentiating $h\left(n, \tau_{b}\right)=0$ along $\tau_{a}$, we find

$$
\left(\nabla_{\tau_{a}} h\right)\left(n, \tau_{b}\right)=-h\left(\nabla_{\tau_{a}} n, \tau_{b}\right)-h\left(n, \nabla_{\tau_{a}} \tau_{b}\right) .
$$

The second fundamental form $\mathscr{K}\left(\tau, \tau^{\prime}\right)$ of $\Gamma_{t}$ in $(D(t)$, eucl) (equivalently, in $(D(t), g))$ is defined by

$$
d_{\tau_{a}} \tau_{b}=\nabla_{\tau_{a}}^{\Gamma} \tau_{b}+\mathscr{K}\left(\tau_{a}, \tau_{b}\right) n \quad \text { on } \partial D(t) .
$$

To relate $\mathscr{K}$ to $h_{\mid \partial D(t)}$, note that since $w=0$ on $\partial D(t)$ we have

$$
h\left(\tau_{a}, \tau_{b}\right)=\frac{1}{v} d^{2} w\left(\tau_{a}, \tau_{b}\right)=\frac{1}{v}\left(\tau_{a}\left(\tau_{b} w\right)-D w \cdot d_{\tau_{a}} \tau_{b}\right)=-d_{\tau_{a}} \tau_{b} \cdot \frac{D w}{v}=-\beta_{0} \mathscr{K}\left(\tau_{a}, \tau_{b}\right) .
$$


(So we see that $\Gamma_{t}$ convex with respect to $n$ corresponds to $\Sigma_{t}$ concave over $D(t)$, as expected.) In (B-1) in the appendix we observe that $\nabla_{\partial_{i}} \partial_{j}=\left(h_{i j} / v\right) D w$. Then

$$
\begin{aligned}
\nabla_{\tau_{a}} \tau_{b} & =\tau_{a}^{i}\left(\left(\tau_{b}^{j}\right)_{i} \partial_{j}+\tau_{b}^{j} \nabla_{\partial_{i}} \partial_{j}\right)=d_{\tau_{a}} \tau_{b}+\frac{1}{v} \tau_{a}^{i} \tau_{b}^{j} h_{i j} D w \\
& =\nabla_{\tau_{a}}^{\Gamma} \tau_{b}+\mathscr{K}\left(\tau_{a}, \tau_{b}\right) n+\frac{w_{n}}{v} h\left(\tau_{a}, \tau_{b}\right) n=\left(-\frac{1}{\beta_{0}}+\beta_{0}\right) h\left(\tau_{a}, \tau_{b}\right) n=-\frac{\beta^{2}}{\beta_{0}} h\left(\tau_{a}, \tau_{b}\right) n
\end{aligned}
$$

at $p$, given our assumption $\nabla_{\tau_{a}}^{\Gamma} \tau_{b}(p)=0$. We use this immediately to compute, at $p$,

$$
\nabla_{\tau_{a}} n=\left\langle\nabla_{\tau_{a}} n, \tau_{b}\right\rangle_{g} \tau_{b}=-\left\langle n, \nabla_{\tau_{a}} \tau_{b}\right\rangle_{g} \tau_{b}=\frac{\beta^{2}}{\beta_{0}}|n|_{g}^{2} h\left(\tau_{a}, \tau_{b}\right) \tau_{b}=\frac{1}{\beta_{0}} h\left(\tau_{a}, \tau_{b}\right) \tau_{b},
$$

since $|n|_{g}^{2}=g_{i j} n^{i} n^{j}=1+w_{n}^{2}=\beta^{-2}$ at $p$. Using these expressions for $\nabla_{\tau_{a}} n$ and $\nabla_{\tau_{a}} \tau_{b}$ in $(11-3)$ and recalling the Codazzi equations, we obtain

$$
\left(\nabla_{n} h\right)\left(\tau_{a}, \tau_{b}\right)=\left(\nabla_{\tau_{a}} h\right)\left(n, \tau_{b}\right)=-\frac{1}{\beta_{0}} \sum_{c} h\left(\tau_{a}, \tau_{c}\right) h\left(\tau_{c}, \tau_{b}\right)+\frac{\beta^{2}}{\beta_{0}} h\left(\tau_{a}, \tau_{b}\right) h_{n n} .
$$

This can also be written in the form

$$
\beta_{0}\left(\nabla_{n} h\right)\left(\tau, \tau^{\prime}\right)=-\left(h^{\tan }\right)^{2}\left(\tau, \tau^{\prime}\right)+\beta^{2} h_{n n} h\left(\tau, \tau^{\prime}\right) .
$$

It turns out the expression for the $n$-directional derivative of $h\left(\tau, \tau^{\prime}\right)$ is exactly the same (at $\partial D(t)$ ):

$$
\beta_{0} d_{n}\left(h\left(\tau, \tau^{\prime}\right)\right)=-\left(h^{\tan }\right)^{2}\left(\tau, \tau^{\prime}\right)+\beta^{2} h_{n n} h\left(\tau, \tau^{\prime}\right) .
$$

The reason is that $\nabla_{n} \tau_{a}=0$ at the boundary, also for the $g$-connection

$$
\nabla_{n} \tau_{a}=d_{n}\left(\tau_{a}\right)+n^{i} \tau_{a}^{j} \nabla_{\partial_{i}} \partial_{j}=0+\frac{1}{v} h\left(n, \tau_{a}\right) D w=0
$$

so in fact

$$
\left(\nabla_{n} h\right)\left(\tau_{a}, \tau_{b}\right)=n\left(h\left(\tau_{a}, \tau_{b}\right)\right)=d_{n}\left(h\left(\tau_{a}, \tau_{b}\right)\right) .
$$

As done in [Stahl 1996], we combine this with the result for $H_{n}$ to compute $\left(\nabla_{n} h\right)(n, n)$. From

$$
H_{n}=\nabla_{n}\left(\operatorname{tr}_{g} h\right)=\operatorname{tr}_{g}\left(\nabla_{n} h\right)=\beta^{2}\left(\nabla_{n} h\right)(n, n)+\sum_{a}\left(\nabla_{n} h\right)\left(\tau_{a}, \tau_{a}\right)
$$

Here we used $|n|_{g}^{2}=\beta^{-2}$ on $\partial D(t)$, which also implies $H=\beta^{2} h_{n n}+\sum_{a} h\left(\tau_{a}, \tau_{a}\right)$. Using also $\left|h^{\tan }\right|^{2}=$ $\sum\left(h^{\tan }\right)^{2}\left(\tau_{a}, \tau_{a}\right)$, we find for $\left(\nabla_{n} h\right)(n, n)$

$$
\beta^{2}\left(\nabla_{n} h\right)(n, n)=\frac{\beta^{2}}{\beta_{0}} H h_{n n}+\frac{1}{\beta_{0}}\left|h^{\tan }\right|^{2}-\frac{\beta^{2}}{\beta_{0}}\left(H-\beta^{2} h_{n n}\right) h_{n n}=\frac{1}{\beta_{0}}\left(\left|h^{\tan }\right|^{2}+\beta^{4} h_{n n}^{2}\right)=\frac{1}{\beta_{0}}|h|_{g}^{2},
$$

since $g^{n n}=\beta^{2}$ at $\partial D(t)$. Equivalently,

$$
\beta_{0}\left(\nabla_{n} h\right)(n, n)=\frac{1}{\beta^{2}}|h|_{g}^{2} \text { on } \partial D(t) .
$$

It is easy to obtain the corresponding expression for the euclidean connection. Noting that

$$
\nabla_{n} n=d_{n} n+n^{i} n^{j} \frac{1}{v} h_{i j} D w=\beta_{0} h_{n n} n \quad \text { at } \partial D(t)
$$


we find

$$
\left(d_{n} h\right)(n, n)=n\left(h_{n n}\right)=\left(\nabla_{n} h\right)(n, n)+2 h\left(\nabla_{n} n, n\right)=\left(\nabla_{n} h\right)(n, n)+2 \beta_{0} h_{n n}^{2}
$$

so that

$$
\beta_{0} d_{n}(h(n, n))=\frac{1}{\beta^{2}}|h|_{g}^{2}+2 \beta_{0}^{2} h_{n n}^{2} \text { on } \partial D(t) .
$$

We record these results as a lemma, including also the evolution equations derived in Appendix B.

Lemma 11.2. Under graph mean curvature motion with constant contact angle, the second fundamental form satisfies the following tensorial evolution equations, where $C_{i j}$ and $\bar{C}_{i j}$ are symmetric 2-tensors cubic in $h$; see (B-2) and (B-3). Recall that $\omega=D w / v$ and $d_{\omega}$ denotes directional derivative.

(i) For the operator $L=L_{g}$,

$$
L\left[h_{i j}\right]=-2\left[h_{i}^{k} d_{\omega}\left(h_{j k}\right)+h_{j}^{k} d_{\omega}\left(h_{i k}\right)\right]+\bar{C}_{i j} \quad \text { on } D(t),
$$

with boundary conditions on $\partial D(t)$ given by

$$
\left\{\begin{array}{l}
h(n, \tau)=0, \\
\beta_{0} d_{n}\left(h\left(\tau, \tau^{\prime}\right)\right)=-\left(h^{\mathrm{tan}}\right)^{2}\left(\tau, \tau^{\prime}\right)+\beta^{2} h_{n n} h\left(\tau, \tau^{\prime}\right), \\
\beta_{0} d_{n}(h(n, n))=|h|_{g}^{2} / \beta^{2}+2 \beta_{0}^{2} h_{n n}^{2} .
\end{array}\right.
$$

(ii) For the operator $\partial_{t}-\Delta_{g}$, where $\Delta_{g}$ is the Laplace-Beltrami operator of $g$

$$
\left(\partial_{t}-\Delta_{g}\right)[h]_{i j}=H\left(\nabla_{\omega} h\right)_{i j}+H_{i} h\left(\omega, \partial_{j}\right)+H_{j} h\left(\omega, \partial_{i}\right)+C_{i j} \quad \text { on } D(t),
$$

with boundary conditions on $\partial D(t)$ given by

$$
\left\{\begin{array}{l}
h(n, \tau)=0, \\
\beta_{0}\left(\nabla_{n} h\right)\left(\tau, \tau^{\prime}\right)=-\left(h^{\tan }\right)^{2}\left(\tau, \tau^{\prime}\right)+\beta^{2} h_{n n} h\left(\tau, \tau^{\prime}\right) \\
\beta_{0}\left(\nabla_{n} h\right)(n, n)=|h|_{g}^{2} / \beta^{2}
\end{array}\right.
$$

It is also useful to compute the boundary condition for $|h|_{g}^{2}$. Using Lemma 11.2(ii), we have at $\partial D(t)$

$$
\begin{aligned}
\left(\beta_{0} / 2\right) d_{n}|h|_{g}^{2} & =\beta_{0}\left\langle\nabla_{n} h, h\right\rangle_{g} \\
& =\beta_{0} \beta^{4}\left(\nabla_{n} h\right)(n, n) h_{n n}+\beta_{0} \sum_{b, c}\left(\nabla_{n} h\right)\left(\tau_{a}, \tau_{b}\right) h\left(\tau_{a}, \tau_{b}\right) \\
& =\beta^{2}|h|_{g}^{2} h_{n n}+\sum_{a, b}\left[-\left(h^{\tan }\right)^{2}\left(\tau_{a}, \tau_{b}\right)+\beta^{2} h_{n n} h\left(\tau_{a}, \tau_{b}\right)\right] h\left(\tau_{a}, \tau_{b}\right) \\
& =\beta^{2}\left(|h|_{g}^{2}+\left|h^{\tan }\right|_{g}^{2}\right) h_{n n}-\operatorname{tr}_{g}\left(h^{\tan }\right)^{3}
\end{aligned}
$$

Since $\operatorname{tr}_{g} h^{3}=\beta^{6}\left(h_{n n}\right)^{3}+\operatorname{tr}_{g}\left(h^{\tan }\right)^{3}$ on $\partial D(t)$, we may state this in a slightly different form. Including also the evolution equation for $|h|_{g}^{2}$ (see Appendix B), we have the following lemma:

Lemma 11.3. Under graph mean curvature flow, the function $|h|_{g}^{2}$ satisfies the evolution equation and Neumann boundary condition

$$
\left\{\begin{array}{l}
\left(\partial_{t}-\Delta_{g}\right)|h|_{g}^{2}=-2|\nabla h|_{g}^{2}+H d_{\omega}|h|_{g}^{2}+2|h|_{g}^{4}-4 H h^{3}(\omega, \omega)-2 H|h|_{g}^{2} h(\omega, \omega) \\
\left(\beta_{0} / 2\right) d_{n}|h|_{g}^{2}=2 \beta^{2}|h|_{g}^{2} h_{n n}-\operatorname{tr}_{g}\left(h^{3}\right) \quad \text { on } \partial D(t) .
\end{array}\right.
$$




\section{A maximum principle for symmetric 2-tensors}

By the local existence theorem, for suitable initial data we have a mean curvature motion $F=[\varphi, u] \in$ $C^{2+\alpha, 1+\alpha / 2}\left(Q_{0}, \mathbb{R}^{n+1}\right)$, where $Q_{0}=D_{0} \times[0, T]$ and, for each $t \in[0, T], \varphi_{t}: D_{0} \rightarrow D(t)$ is a $C^{2+\alpha}$ diffeomorphism. In particular, with $\delta=\alpha^{2}, w_{t}=u_{t} \circ \varphi_{t}^{-1}: D(t) \rightarrow \mathbb{R}$ defines a graph mcm $w \in$ $C^{2+\delta, 1+\delta / 2}(E ; \mathbb{R})$ in an open spacetime domain

$$
E=\bigcup_{t \in(0, T)} D(t) \times\{t\} \subset \mathbb{R}^{n} \times \mathbb{R}
$$

We have a $C^{2+\alpha, 1+\alpha / 2}$ diffeomorphism

$$
\Phi: \bar{Q}_{0} \rightarrow \bar{E}, \quad \Phi(x, t)=\left(\varphi_{t}(x), t\right),
$$

which, for any $t_{0}>0$, restricts to a diffeomorphism $Q_{t_{0}} \rightarrow E_{t_{0}}$, where

$$
Q_{t_{0}}=D_{0} \times\left(t_{0}, T\right), \quad E_{t_{0}}=\bigcup_{t \in\left(t_{0}, T\right)} D(t) \times\{t\} .
$$

The parabolic boundary of $E$ is the disjoint union of base and lateral boundary:

$$
\partial_{p} E=\left(\bar{D}_{0} \times\{0\}\right) \sqcup \partial_{l} E, \quad \partial_{l} E=\bigcup_{t \in(0, T)} \partial D(t) \times\{t\} .
$$

(The notions of parabolic boundary, base and lateral boundary have general definitions for arbitrary bounded spacetime domains [Lieberman 1996], but using $\Phi$ it is easy to see that they are given by the above sets.) In particular, note that $\Phi$ defines a diffeomorphism

$$
Q_{t_{0}} \cup \partial_{l} Q_{t_{0}} \rightarrow E_{t_{0}} \cup \partial_{l} E_{t_{0}},
$$

for each $t_{0}>0$. This diffeomorphism is $C^{k+\alpha,(k+\alpha) / 2}$ up to the lateral boundary, if $D_{0}$ is a $C^{k+\alpha}$ domain and $F \in C^{k+\alpha,(k+\alpha) / 2)}\left(Q_{0}\right)$.

Denote by $L$ the operator $L=\partial_{t}-g^{i j}(D w) \partial_{i} \partial_{j}$, so $L w=0$ in $E$ and $w=0$ on $\partial_{l} E$. The following height bound is immediate.

Lemma 12.1. Assume $0<w_{0}<M$ in $D_{0}$. Then $0 \leq w \leq M$ in $\bar{E}$ (and vanishes only on $\partial_{l} E$ ).

Proof. This follows from the weak maximum principle for the operator $L$, since $0 \leq w \leq M$ holds on the parabolic boundary $\partial_{p} E$.

It is well-known that the function $v=\sqrt{1+|D w|^{2}}$ solves the evolution equation (assuming $D w \in$ $C^{2,1}(\bar{E})$ - see [Guan 1996], for example)

$$
L[v]+\frac{2}{v} g^{i j} v_{i} v_{j}=-v|h|_{g}^{2}, \text { or } L[v]=-\frac{2}{v}|D v|_{g}^{2}-v|h|_{g}^{2} .
$$

From the maximum principle, we have the following global bound on $v$ (equivalently, on $|D w|$ ):

Lemma 12.2. Assume $w$ is a solution with $D w \in C^{2,1}(\bar{E})$. Then, on $\bar{E}$,

$$
v(z) \leq \max \left\{\sup _{D\left(t_{0}\right)} v\left(x, t_{0}\right), 1 / \beta\right\} .
$$

Proof. By the weak maximum principle, $\max _{\bar{E}} v=\max _{\partial_{p} E} v$. Note that $v_{\mid S} \equiv 1 / \beta$. 
It follows from this lemma that $g_{i j}(t)$ is uniformly equivalent to the euclidean metric in $D(t)$ : If $v \leq \bar{v}$ in $\bar{E}$, and $X$ is a vector field in $D(t)$, then

$$
|X|_{e}^{2} \leq|X|_{g}^{2}=g_{i j} X^{i} X^{j}=|X|_{e}^{2}+(X \cdot D w)^{2} \leq|X|_{e}^{2}\left(1+|D w|^{2}\right) \leq \bar{v}^{2}|X|_{e}^{2} .
$$

Also, if $\omega:=v^{-1} D w$ then

$$
|\omega|_{e}^{2}=\frac{|D w|_{e}^{2}}{v^{2}}=1-\frac{1}{v^{2}} \leq 1-\frac{1}{\bar{v}^{2}} .
$$

The main result in this section is a maximum principle for symmetric 2-tensors satisfying a parabolic equation on a spacetime domain such as $E$ (image of a cylinder under a diffeomorphism of the special type $\Phi)$.

We recall the boundary point lemma for scalar equations, which holds for open spacetime domains $\Omega \subset \mathbb{R}^{n} \times \mathbb{R}_{+}$satisfying an interior ball condition:

For each $P=(p, \bar{t}) \in \partial_{l} \Omega$ there is a ball $B$ (in the euclidean metric in $\mathbb{R}^{n+1}$ ) which is tangent to $\partial_{l} \Omega$ only at $P$ and satisfies:

(i) The line segment from $P$ to the center of the ball is not parallel to the $t$ axis.

(ii) $B \cap\{t \leq \bar{t}\} \subset \Omega \cap\{t \leq \bar{t}\}$.

For the domain of interest the interior ball condition follows from the fact that $\partial_{l} E=\Phi\left(\partial D_{0} \times(0, T)\right)$, with $\Phi \in C^{2,1}\left(D_{0} \times(0, T)\right)$ of the special form above.

Lemma 12.3 [Protter and Weinberger 1984, Theorem 6, page 174]. Let $\Omega \subset \mathbb{R}^{n} \times \mathbb{R}_{+}$be a connected open set satisfying the interior ball condition. Assume $f \in C^{2,1}(\Omega)$ satisfies the uniformly parabolic inequality

$$
\partial_{t} f-\operatorname{tr}_{g} d^{2} f-d_{X} f \leq 0
$$

Here $g=g_{t}$ is a Riemannian metric in each section $\Omega(t)$, and $X_{t}$ is a bounded vector field in $\Omega(t)$. Denote by $n=n_{t}$ the inner unit normal of $\Omega(t)$. Assume the supremum $M$ of $f$ in $\Omega_{\bar{t}}:=\Omega \cap\{t \leq \bar{t}\}$ is attained at the point $P \in \partial \Omega(\bar{t})$, and that $f<M$ for $t<\bar{t}$. Then $d_{n} f(P)<0$.

We now state the hypotheses of our tensorial maximum principle.

$E \subset \mathbb{R}^{n} \times[0, T]$ is the image of a cylinder $D_{0} \times(0, T)$ under a $C^{3,2}$ diffeomorphism $\Phi$ of the form $\Phi(x, t)=\left(\varphi_{t}(x), t\right)$ with $\varphi_{t}: D_{0} \rightarrow D(t)$ a $C^{3}$ diffeomorphism up to the boundary for each $t \in[0, T]$ (here $D(t)$ is the $t$-level set of $E$ ); $\bar{D}_{0} \subset \mathbb{R}^{n}$ is assumed to be the image of the closed unit ball under a $C^{3}$ diffeomorphism. In particular, the lateral boundary $\partial_{l} E$ is of class $C^{3,2}$. On $\partial_{l} E$ we have the inner unit normal $n=n_{t} \in \mathbb{R}^{n}$. Extend $n_{t}$ to a vector field in all of $\bar{D}(t)$ so that it is in $C^{2,1}\left(\bar{E}, \mathbb{R}^{n}\right)$, arbitrarily except for the requirements that $|n| \leq 1$ pointwise and $d_{n} n=0$ in a tubular neighborhood of $\partial D(t)$ (equivalently, $n^{i} \partial_{i} n^{j}=0$ for each $j$ ). Fix $R>0$ so that $D(t) \subset B_{R}(0)$ for each $t \in[0, T]$.

The assumptions on the coefficients are given next:

- $g=g_{t}$ is a $t$-dependent Riemannian metric in $\bar{D}(t)$, uniformly equivalent to the euclidean metric for $t \in[0, T]$;

- $X=X_{t}$ is a bounded $t$-dependent vector field in $\bar{D}(t)$;

- $q=q(z, m)$ assigns to each $z \in \bar{E}$ and each $m$ in $\mathbb{S}$ (the space of quadratic forms in $\mathbb{R}^{n}$ ) a quadratic form $q \in \mathbb{S} . q$ is assumed to be $C^{2,1}$ in $z$, locally Lipschitz in $m$ (uniformly in $z \in \bar{E}$ );

- $b=b(z, m) \in \mathbb{S}$ is defined for $z \in \partial_{l} E$, with the same regularity assumptions as $q$. 
We state the next theorem in terms of the Laplace-Beltrami heat operator $\partial_{t}-\Delta_{g}$ and the $g$-Riemannian connection $\nabla$, but the result also holds for $L$ and the "euclidean connection" $d$.

Theorem 12.4. Assume $m \in C^{2,1}(\bar{E} ; S)$ satisfies in $E$ the tensorial differential inequality

$$
\partial_{t} m_{i j}-\left(\Delta_{g} m\right)_{i j} \leq\left(\nabla_{X} m\right)_{i j}+q_{i j}(\cdot, m(\cdot)),
$$

and on $\partial_{l} E$ the boundary condition

$$
\left(\nabla_{n} m\right)_{i j}(z) \geq b_{i j}(z, m(z)) .
$$

Suppose the functions $q$ and $b$ satisfy the following null eigenvector conditions: for any $\hat{m} \in \mathbb{S}$ and any null eigenvector $V \in \mathbb{R}^{n}$ of $\hat{m}$ (meaning that $\hat{m}_{i j} V^{j}=0$ for all $i$ ), we have $q_{i j}(z, \hat{m}) V^{i} V^{j} \leq 0$ for all $z \in \bar{E}$ and $b_{i j}(z, \hat{m}) V^{i} V^{j} \geq 0$ for all $z \in \partial_{l} E$. Then weak concavity of $m$ at $t=0$ is preserved:

$$
m \leq 0 \text { in } D(0) \Longrightarrow m \leq 0 \text { in } \bar{E} \text {. }
$$

Proof. The assumptions imply that there is a $K>0$ (depending only on $E$ and on the functions $X, g, n$, $q$, and $b$ ) satisfying

$$
|n|_{C^{2,1}(\bar{E})} \leq K, \quad|X(z)|_{\mathrm{eucl}} \leq K, \quad|g(z)|+\left|g^{-1}(z)\right| \leq K, \quad z \in \bar{E},
$$

and if $m, \hat{m} \in C^{2,1}\left(\bar{E}, \mathbb{S}\right.$ ) satisfy (for some $\mu: \bar{E} \rightarrow \mathbb{R}_{+}$)

$$
-\mu(z) g \leq m(z)-\hat{m}(z) \leq \mu(z) g
$$

(where the inequality of quadratic forms has the usual meaning) then also

$$
\begin{array}{ll}
q(z, m(z)) \leq q(z, \hat{m}(z))+K \mu(z) g, & z \in \bar{E}, \\
b(z, m(z)) \geq b(z, \hat{m}(z))-K \mu(z) g, & z \in \partial_{l} E .
\end{array}
$$

Now, for $z \in \bar{E}, z=(x, t)$ define

$$
\varphi(z):=-2 K n(z) \cdot x:=2 K s(z)
$$

where we use the euclidean inner product and, on $\partial_{l} E, s$ is the "support function" of $\partial D(t)$ (positive if $D(t)$ is convex and contains the origin). It is clear that we can find $M=M(R, K)>0$ depending only on $K, R$ and $|n|_{C^{2,1}}$ so that

$$
|\varphi|_{C^{2,1}} \leq M, \quad|d \varphi|_{g}^{2}+\left|\Delta_{g} \varphi\right| \leq M, \quad|X \cdot d \varphi| \leq M
$$

We assume also $M \geq K$. Now, given $m$ as in the statement of the theorem and given constants $\epsilon>0, \gamma>0$, and $\delta>0$, define for $E^{\delta}:=E \cap\{t<\delta\}$

$$
\hat{m}(z):=m(z)-\left(\epsilon t+\gamma e^{\varphi(z)}\right) g, \quad z \in \bar{E}^{\delta} .
$$

Clearly $\hat{m} \in C^{2,1}\left(\bar{E}^{\delta} ; \mathbb{S}\right)$. We now derive the constraints on $\delta, \epsilon$, and $\gamma$. It will turn out that $\delta$ must be taken small enough (depending only on $K, R), \epsilon>0$ is arbitrary, and $\gamma$ is $\epsilon$ times a constant depending only on $K, R$. 
The following inequalities are easily derived:

$$
\begin{gathered}
q(z, m(z)) \leq q(z, \hat{m}(z))+K\left(\epsilon t+\gamma e^{\varphi(z)}\right) g, \\
\nabla_{X} m=\nabla_{X} \hat{m}+\gamma\left(e^{\varphi} d_{X} \varphi\right) g \leq \nabla_{X} \hat{m}+\left(\gamma e^{\varphi} M\right) g, \\
\partial_{t} \hat{m}=\partial_{t} m-\epsilon g-\left(\gamma e^{\varphi} \partial_{t} \varphi\right) g \leq \partial_{t} m+\left(\gamma e^{\varphi} M\right) g-\epsilon g, \\
\Delta_{g} \hat{m}=\Delta_{g} d^{2} \hat{m}-\gamma e^{\varphi}\left(|d \varphi|_{g}^{2}+\Delta_{g} \varphi\right) g \geq \Delta_{g} m-\left(\gamma e^{\varphi} M\right) g, \\
b(z, m(z)) \geq b(z, \hat{m}(z))-K\left(\epsilon t+\gamma e^{\varphi}\right) g .
\end{gathered}
$$

We use this to compute

$$
\begin{aligned}
\partial_{t} \hat{m}-\Delta_{g} \hat{m} & \leq \partial_{t} m-\Delta_{g} m+\left(2 \gamma e^{\varphi} M\right) g-\epsilon g \\
& \leq q(z, m(z))+\nabla_{X} m+\left(2 \gamma e^{\varphi} M\right) g-\epsilon g \\
& \leq q(z, \hat{m}(z))+\nabla_{X} \hat{m}+K\left(\epsilon t+\gamma e^{\varphi}\right) g+\left(3 M \gamma e^{\varphi}\right) g-\epsilon g \\
& \leq q(z, \hat{m}(z))+\nabla_{X} \hat{m}+M \epsilon t g+4 M \gamma e^{\varphi} g-\epsilon g,
\end{aligned}
$$

using $K \leq M$ in the last step. We conclude the inequality

$$
\partial_{t} \hat{m}-\Delta_{g} \hat{m} \leq q(z, \hat{m}(z))+\nabla_{X} \hat{m}-(\epsilon / 2) g
$$

will hold in $E^{\delta}$, provided the constants are selected so that, for $z \in E^{\delta}$

$$
4 M \gamma e^{\varphi(z)}+M \epsilon t \leq \epsilon / 2 .
$$

Turning to boundary points $z=(x, t) \in \partial_{l} E$, note that $d_{n} \varphi=-2 K$, so that

$$
\begin{aligned}
\nabla_{n} \hat{m}(z) & =\nabla_{n} m(z)-\left(\gamma e^{\varphi(z)} d_{n} \varphi(z)\right) g \\
& \geq b(z, m(z))-\left(\gamma e^{\varphi(z)} d_{n} \varphi(z)\right) g \\
& \geq b(z, \hat{m}(z))-K\left(\epsilon t+\gamma e^{\varphi(z)}\right) g-\left(\gamma e^{\varphi(z)} d_{n} \varphi(z)\right) g \\
& \geq b(z, \hat{m}(z))+K\left(\gamma e^{\varphi(z)}-\epsilon t\right) g,
\end{aligned}
$$

implying the inequality

$$
\nabla_{n} \hat{m}(z) \geq b(z, \hat{m}), \quad z \in \partial_{l} E^{\delta}
$$

will hold provided the constants are so chosen that, on $\partial_{l} E^{\delta}$

$$
\epsilon t \leq \gamma e^{\varphi(z)} \text {. }
$$

Bearing in mind that $e^{-2 K R} \leq e^{\varphi(z)} \leq e^{2 K R}$ on $E$, it is not hard to arrange for (12-2) and (12-4) to hold, or equivalently, for

$$
\epsilon t \leq \gamma e^{\varphi(z)}, \quad 10 M \gamma e^{\varphi(z)} \leq \epsilon .
$$

Given $\epsilon>0$, define $\gamma$ so that $10 M \gamma e^{2 K R}=\epsilon$. Then the second inequality holds, and so will the first, provided that

$$
\epsilon t \leq \gamma e^{-2 K R}=(\epsilon / 10 M) e^{-4 K R},
$$

which is true for any $\epsilon>0$, if $\delta$ is defined by $\delta:=e^{-4 K R} / 10 M$ (recall $t \in[0, \delta]$ ). 
Note that, since $m \leq 0$ at $t=0$, it follows that $\hat{m}$ is negative definite at $t=0$, and hence also for small time, and we claim that this persists throughout $\bar{E}^{\delta}$ so that (letting $\left.\epsilon \rightarrow 0\right) m \leq 0$ in $\bar{E}^{\delta}$. Restarting the argument at $t=\delta$, we see that this is enough to prove the theorem.

To prove the claim, suppose for a contradiction that $\hat{m}$ acquires a null eigenvector $0 \neq V \in \mathbb{R}^{n}$ at a point $z_{1}=\left(x_{1}, t_{1}\right) \in \bar{E}^{\delta}$ with $t_{1} \in(0, \delta]$ the first time this happens.

Let $\hat{f}(z):=\hat{m}_{i j} V^{i} V^{j}$ for $z \in E^{\delta}$ (that is, we "extend" $V$ to $E^{\delta}$ as a constant vector). It follows from (12-1) that $\hat{f}$ satisfies in $E^{\delta}$

$$
\partial_{t} \hat{f} \leq\left(\Delta_{g} \hat{m}\right)_{i j} V^{i} V^{j}+\left(\nabla_{X} \hat{m}\right)_{i j} V^{i} V^{j}+q_{i j}(\cdot, \hat{m}) V^{i} V^{j}-\frac{1}{2} \epsilon|V|_{g}^{2} .
$$

A short, standard Riemannian calculation using the fact that $V$ is a null eigenvector for $\hat{m}$ shows that

$$
d_{X} \hat{f}=\left(\nabla_{X} \hat{m}\right)_{i j} V^{i} V^{j}, \quad \Delta_{g} \hat{f}=\left(\Delta_{g} \hat{m}\right)_{i j} V^{i} V^{j} .
$$

Using the null eigenvector condition for $q$, we find that $\hat{f}$ satisfies in $E^{\delta}$ the strict inequality

$$
\partial_{t} \hat{f}<\operatorname{tr}_{g} d^{2} \hat{f}+d_{X} \hat{f}
$$

This shows $x_{1}$ cannot be an interior point of $D\left(t_{1}\right)$, for then (as a first-time interior maximum point for $\hat{f}$ ) we would have $\Delta_{g} \hat{f}\left(z_{1}\right) \leq 0$ and $d \hat{f}\left(z_{1}\right)=0$, contradicting $\partial_{t} \hat{f}\left(z_{1}\right) \geq 0$. Thus $x_{1} \in \partial D\left(t_{1}\right)$. Since $\hat{f}$ satisfies the differential inequality just stated and $z_{1}=\left(x_{1}, t_{1}\right)$ is a first-time boundary maximum in $\bar{E}^{\delta}$, the parabolic Hopf lemma (Lemma 12.3) implies $d_{n} \hat{f}\left(z_{1}\right)<0$. On the other hand, as seen in (12-3),

$$
d_{n} \hat{f}=\left(\nabla_{n} \hat{m}\right)_{i j} V^{i} V^{j} \geq b_{i j}\left(z_{1}, \hat{m}\left(z_{1}\right)\right) V^{i} V^{j} \geq 0
$$

from the null eigenvector condition on the boundary. This contradiction concludes the proof.

Corollary 12.5. Suppose $m \in C^{2,1}(\bar{E}, \mathbb{S})$ satisfies the same differential inequality with the same hypotheses on the coefficients as in Theorem 12.4 (including the null eigenvector condition for $q$ ), and the boundary conditions

$$
\left\{\begin{array}{l}
m(z)(n, \tau)=0, \quad \forall z=(x, t) \in \partial_{l} E, \tau \in T_{x} \partial D(t) \\
\left(\nabla_{n} m\right)(n, n) \geq b_{n n}(z, m(z)) \\
\left(\nabla_{n} m\right)(\tau, \tau) \geq b^{\tan }(z, m(z))(\tau, \tau), \quad \tau \in T_{x} \partial D(t),
\end{array}\right.
$$

for functions $b_{n n}(z, \hat{m})$ from $\partial_{l} E \times \mathbb{S}$ to $\mathbb{R}$ and $b^{\tan }$ assigning to $(z, \hat{m}) \in \partial_{l} E \times \mathbb{S}, z=(x, t)$, a quadratic form in $T_{x} \partial D(t)$. Suppose $b_{n n} \geq 0$ in $E \times \mathbb{S}$ and $b^{\tan }$ satisfies, for each $\hat{m} \in \mathbb{S}$,

$$
\hat{m}_{i j} \tau^{i}=0 \text { for some } \tau \in T_{x} \partial D(t) \quad \Longrightarrow \quad b^{\tan }(z, \hat{m})(\tau, \tau) \geq 0 .
$$

Then, as in the theorem, weak concavity is preserved:

$$
m \leq 0 \text { at } t=0 \Longrightarrow m \leq 0 \text { in } \bar{E} .
$$

Proof. As for the theorem, with the following change in the last part of the proof: If $0 \neq V \in \mathbb{R}^{n}$ is a null eigenvector of $\hat{m}$ (defined as in the proof of the theorem) at a boundary point $z_{1}=\left(x_{1}, t_{1}\right) \in \partial_{l} E$, write

$$
V=V^{n} n+V^{T}, \quad V^{T} \in T_{x_{1}} \partial D\left(t_{1}\right) .
$$


Assume first that $V^{n} \neq 0$. Then, noting that $\hat{m}$ splits at the boundary if $m$ does, we see that $n$ is a null eigenvector of $\hat{m}$ at $z_{1}$, so we define $\hat{f}(z)=\hat{m}_{i j}(z) n^{i}\left(z_{1}\right) n^{j}\left(z_{1}\right)$ and repeat the argument. At $z_{1}$, $\left(\nabla_{n} \hat{m}\right)(n, n) \geq b_{n n}\left(z_{1}, \hat{m}\left(z_{1}\right)\right) \geq 0$ leads to a contradiction with the parabolic Hopf lemma, as before.

If $V^{n}=0$, then $V^{T} \in T_{x_{1}} \partial D\left(t_{1}\right)$ must be a null eigenvector of $\hat{m}$ at the boundary point $z_{1}$, and then we run the argument with $\hat{f}(z)=\hat{m}(z)\left(V^{T}, V^{T}\right)$, leading to a contradiction, as before.

Corollary 12.6. Let $w \in C^{4,2}(E)$ define a mcm of graphs with constant-angle boundary conditions, where $E$ is as in the statement of Theorem 12.4. Then weak concavity is preserved:

$$
h \leq 0 \text { at } t=0 \Longrightarrow h \leq 0 \text { in } \bar{E} \text {. }
$$

Proof. From Lemma 11.2, $h$ satisfies $\left(\partial_{t}-\Delta_{g}\right) h_{i j}=H \nabla_{\omega} h_{i j}+q(z, h)_{i j}$ and

$$
q(z, h)_{i j}=H_{i} h\left(\omega, \partial_{j}\right)+H_{j} h\left(\omega, \partial_{i}\right)+|h|_{g}^{2} h_{i j}+H h\left(\partial_{i}, \omega\right) h\left(\partial_{j}, \omega\right)-H h(\omega, \omega) h_{i j},
$$

where $H_{i}, H_{j}, H$ and $\omega^{i}$ are regarded as fixed functions of $z \in E$. Clearly $q$ satisfies the null eigenvector condition, since $q_{i j} V^{i} V^{j}=0$ when $h_{i j} V^{j}=0$ for all $i$. In addition, expressions obtained for $d_{n} h$ in Lemma 11.2 show that the boundary conditions in Corollary 12.5 are satisfied with

$$
b_{n n}(z, \hat{m}) \equiv 0, \quad b^{\tan }(z, \hat{m})=-\left((\hat{m})^{\tan }\right)^{2}+\beta^{2} \hat{m}_{n n} \hat{m}^{\tan }
$$

Hence the claim follows from Corollary 12.5.

For less regular solutions, we may apply the theorem to a domain $E_{t_{0}}=E \cap\left\{t>t_{0}\right\}$ for arbitrarily small $\delta>0$. Thus, assuming $h<0$ at $t=0$ (strictly negative definite), we conclude from Corollary 12.6 that $h \leq 0$ for all $t$.

Remark. It seems plausible that a slightly different version of the result in this section could be used to strengthen the conclusions in [Stahl 1996].

Finite existence time. It is not difficult to derive that the flow is defined only for finite time in the concave case.

Lemma 12.7. Let $w \in C^{4,2}(E), E \subset \mathbb{R}^{n} \times[0, T)$, define a graph $m c m \Sigma_{t}$ with constant-angle boundary conditions on a moving boundary. Assume that $\Sigma_{0}$ (and hence $\Sigma_{t}$, for all $t$ ) is weakly concave. Assume that $H_{\mid t=0} \leq H_{0}<0$, where $H_{0}$ is a negative constant, and that $T=\sup \{t \in[0, T): D(t) \neq \varnothing\}$. Then $T \leq t_{*}=1 /\left(2 H_{0}^{2} c_{n}\right)$, where $c_{n}>0$ depends only on $n$ and an upper bound for $v$ in $E$.

The proof is based on the evolution equation and boundary condition for $H$ (see Appendix B; we have $\omega=D w / v)$ :

$$
L[H]=|h|_{g}^{2} H+H h^{2}(\omega, \omega)-H^{2} h(\omega, \omega), \quad H_{n}=\left(\beta^{2} / \beta_{0}\right) H h_{n n} .
$$

Since $h^{2}(\omega, \omega) \geq 0,|h|_{g}^{2} \geq(1 / n) H^{2}$ and (given that $\left.h \leq 0\right) h(\omega, \omega) \geq|D w|^{2} H$, we have

$$
L[H] \leq \frac{1}{n} H^{3}+|D w|^{2} H^{3} \leq c_{n} H^{3},
$$

where $c_{n}$ depends on $n$ and on $\sup _{E}|v|$, already known to be finite. Let $\phi(t)$ solve the ODE $\dot{\phi}=$ $c_{n} \phi^{3}, \phi(0)=H_{0}$, so

$$
\phi(t)=H_{0}\left[1-2 c_{n} H_{0}^{2} t\right]^{-1 / 2}, \quad 0 \leq t<t_{*}:=\frac{1}{2 H_{0}^{2} c_{n}} .
$$


Then, with $\psi:=\frac{1}{n}\left(H^{2}+H \phi+\phi^{2}\right)>0$ and setting $\chi=H-\phi$, we have $L[\chi] \leq \psi \chi$ in $E$ and

$$
\chi_{n}=\frac{\beta^{2}}{\beta_{0}}(\chi+\phi) h_{n n} \geq \frac{\beta^{2}}{\beta_{0}} \chi \text { on } \partial_{l} E,
$$

since $\phi<0$ and $h_{n n} \leq 0$. Given that $\chi \leq 0$ at $t=0$, it follows from the maximum principle that $\chi \leq 0$, or $H \leq \phi$ in $\left[0, \min \left\{T, t_{*}\right\}\right)$. This shows $t_{*}<T$ is impossible, since $\phi \rightarrow-\infty$ as $t \rightarrow t_{*}$.

Remark 12.8. It would be natural to try to show that a negative upper bound $H_{0}$ on the mean curvature (at $t=0$ ) is preserved, at least under the assumption of concavity. Unfortunately, the evolution equation for $H$ (under graph $\mathrm{mcm}$ ) does not lend itself to a maximum principle argument. Letting $u:=H-H_{0}$, we have

$$
L[u]=|h|_{g}^{2} u+u h^{2}(\omega, \omega)-u\left(H+H_{0}\right) h(\omega, \omega)+H_{0} Q \quad \text { in } E,
$$

with

$$
Q:=|h|_{g}^{2}+h^{2}(\omega, \omega)-H_{0} h(\omega, \omega) .
$$

At a point where $u=0$, we would need to show $L[u] \leq 0$. But it is not true that $Q \geq 0$ at such a point. (Note that $u_{n} \geq 0$ does hold at boundary points.)

The exception is if $n=2$ (under an additional condition). Let $\hat{\omega}=\omega /|\omega|_{g}, \tilde{\omega}=\omega^{\perp} /|\omega|_{g}$. It is easy to check that $\mathscr{B}=\{\hat{\omega}, \tilde{\omega}\}$ is a $g$-orthonormal frame at each point where $\omega \neq 0$. Then with

$$
a:=h(\hat{\omega}, \hat{\omega}), \quad b:=h(\hat{\omega}, \tilde{\omega}), \quad c:=h(\tilde{\omega}, \tilde{\omega}),
$$

we have

$$
h^{2}(\hat{\omega}, \hat{\omega})-H h(\hat{\omega}, \hat{\omega})=a^{2}+b^{2}-(a+c) a=b^{2}-a c=-\Delta,
$$

where $\Delta$, the determinant of the matrix of $h$ in $\mathscr{B}$, is nonnegative if $h \leq 0$. In particular,

$$
h^{2}(\omega, \omega)-H h(\omega, \omega)=-|\omega|_{g}^{2} \Delta \leq 0
$$

in the concave case. Now consider the expression (12-5) for $Q$, at a point where $u=0$, or $H=H_{0}$. Since $|\omega|_{g}^{2}=|D w|^{2}$ we can write

$$
\begin{aligned}
Q & =|h|_{g}^{2}+h^{2}(\omega, \omega)-H h(\omega, \omega) \\
& =a^{2}+2 b^{2}+c^{2}+|D w|^{2}\left(b^{2}-a c\right) \\
& =b^{2}\left(2+|D w|^{2}\right)+a^{2}-|D w|^{2} a c+c^{2},
\end{aligned}
$$

so $Q \geq 0$ provided $|D w|^{2} \leq 2$. This last condition is equivalent to $v \leq \sqrt{3}$, and hence (Lemma 12.2) is preserved by the evolution if it holds at $t=0$. Thus:

Proposition 12.9. Assume $n=2, h \leq 0$, and $v \leq \sqrt{3}$ on $\Sigma_{0}$ (in particular, $\beta \geq 1 / \sqrt{3}$ ). Then $H \leq H_{0}<0$ at $t=0$ implies $H \leq H_{0}$ for all $t \in\left[0, T_{\max }\right)$.

\section{Global bounds from boundary bounds for $\nabla^{n} h$}

In this section we begin to develop a continuation criterion for solutions of graph mean curvature motion with constant contact angle based on the second fundamental form. Our first observation is that the supremum of $|h|_{g}$ on the moving boundary controls its value in the interior. Recall we already have a bound on $\sup _{E} v$ (Lemma 12.2) and it is a well known-fact for mean curvature flow of graphs that this 
implies interior bounds for the second fundamental form and its covariant derivatives [Ecker and Huisken 1991; Ecker 2004]. In the next lemma we describe a global bound for mean curvature motion of graphs with moving boundaries.

Lemma 13.1. Let $w: E \rightarrow \mathbb{R}$ be a (sufficiently regular) solution of graph mcm in a spacetime domain $E \subset \mathbb{R}^{n} \times[0, T]$, where $T<\infty$. Assume the first derivative bound $v(x, t) \leq \bar{v}$ holds globally in $\bar{E}$. Then if the bound $|h|_{g} \leq h_{0}$ holds on the parabolic boundary $\partial_{p} E$, we also have the global bound

$$
|h|_{g} \leq a_{0} \quad \text { in } \bar{E}
$$

for a constant $a_{0}$ depending only on $n, \bar{v}, h_{0}, T$ and the initial data of $w$.

Proof. The proof is simpler under the assumption that $h$ is negative definite, that is, the concave case. (As shown in the previous section, this condition is preserved if it holds at $t=0$.) We give the details in this case only.

The norms of tensors in $D(t)$ will always be taken with respect to the induced metric $g$, so we write $|h|$ for $|h|_{g},|\nabla h|$ for $|\nabla h|_{g}$, and $|D f|^{2}=g^{i j} f_{i} f_{j}$ for a function $f$.

Recall the evolution equations $L[v]=-v|h|^{2}-2|D v|^{2} / v$ (so $L\left[v^{2}\right]=-2 v^{2}|h|^{2}-6|D v|^{2}$ ) and

$$
L\left[|h|^{2}\right]=-2|\nabla h|^{2}+2|h|^{4}-4 H h^{3}(\omega, \omega)-2 H|h|^{2} h(\omega, \omega) .
$$

In the concave case $H \leq 0$ and $h^{3}$ is negative definite, so we get

$$
L\left[|h|^{2}\right] \leq-2|\nabla h|^{2}+2|h|^{4} .
$$

The idea then is to apply the maximum principle to $f=|h|^{2} v^{2}$. In the evolution equation for $f$,

$$
L[f]=v^{2} L\left[|h|^{2}\right]+|h|^{2} L\left[v^{2}\right]-2\left\langle D|h|^{2}, D v^{2}\right\rangle_{g},
$$

the terms $\pm 2 v^{2}|h|^{4}$ cancel exactly, and we have the inequality

$$
L[f] \leq-2 v^{2}|\nabla h|^{2}-6|h|^{2}|D v|^{2}-2\left\langle D|h|^{2}, D v^{2}\right\rangle_{g} .
$$

The term with the inner product can be estimated in two ways:

$$
\left|\left\langle D|h|^{2}, D v^{2}\right\rangle_{g}\right| \leq\left.\left.|D| h\right|^{2}|| D v^{2}|\leq 4| h|v| \nabla h|| D v\left|\leq 2 v^{2}\right| \nabla h\right|^{2}+2|h|^{2}|D v|^{2}
$$

and

$$
\left\langle D|h|^{2}, D v^{2}\right\rangle_{g}=\frac{1}{v^{2}}\left\langle D\left(|h|^{2} v^{2}\right), D v^{2}\right\rangle_{g}-\frac{|h|^{2}}{v^{2}}\left|D v^{2}\right|^{2}=\frac{1}{v^{2}}\left\langle D f, D v^{2}\right\rangle_{g}-4|h|^{2}|D v|^{2} .
$$

Using the second expression, we have

$$
L[f] \leq-2 v^{2}|\nabla h|^{2}-6|h|^{2}|D v|^{2}-\frac{1}{v^{2}}\left\langle D f, D v^{2}\right\rangle_{g}+4|h|^{2}|D v|^{2}-\left\langle D|h|^{2}, D v^{2}\right\rangle_{g},
$$

and then estimating the remaining inner product term from the first expression

$$
L[f] \leq-2 v^{2}|\nabla h|^{2}-6|h|^{2}|D v|^{2}-\frac{1}{v^{2}}\left\langle D f, D v^{2}\right\rangle_{g}+4|h|^{2}|D v|^{2}+2 v^{2}|\nabla h|^{2}+2|h|^{2}|D v|^{2},
$$

yielding after cancellation

$$
L[f] \leq-\frac{1}{v^{2}}\left\langle D f, D v^{2}\right\rangle_{g}
$$


Applying the (weak) maximum principle to $f$, we conclude

$$
\max _{\bar{E}}|h|^{2} \leq \max _{\bar{E}} f \leq \max _{\partial_{p} E} f \leq \bar{v}^{2} \max _{\partial_{p} E}|h|^{2},
$$

which implies the result (for the concave case) with an explicit constant $a_{0}=\bar{v} h_{0}$.

In the general case, we have

$$
L\left[|h|^{2}\right] \leq-2|\nabla h|^{2}+c_{n}|h|^{4} .
$$

Then the proof follows the same lines as [Ecker 2004, Proposition 3.21]. We apply the maximum principle to $f=|h|^{2}\left(\eta \circ v^{2}\right)$, for a carefully chosen function $\eta(s)$.

Evolution of $|\nabla \boldsymbol{h}|^{2}$. In the calculation that follows, we adopt the usual convention that in symbols such as $\nabla^{2} h *(\nabla h)^{(2)} * h^{(3)}$ and $\left(\nabla^{j} h\right)^{(p)}=\nabla^{j} h * \cdots * \nabla^{j} h(p$ times $)$, $*$ denotes some unspecified $g$ contraction of the tensors in question.

For the time derivative, we have

$$
\begin{aligned}
\partial_{t}|\nabla h|^{2} & =2\left\langle\partial_{t}(\nabla h), \nabla h\right\rangle+\partial_{t}\left(g^{i j} g^{p q} g^{r s}\right)\left(\nabla_{i} h\right)_{p r}\left(\nabla_{j} h\right)_{q s} \\
& =2\left\langle\partial_{t}(\nabla h), \nabla h\right\rangle+3\left(\partial_{t} g^{i j}\right)\left\langle\nabla_{i} h, \nabla_{j} h\right\rangle,
\end{aligned}
$$

using the Codazzi identity.

For the Hessian (using $\nabla_{k} \partial_{l}=h_{k l} \omega$, derived as (B-1) in Appendix B), we get

$$
\begin{aligned}
\nabla_{k, l}^{2}|\nabla h|^{2} & =2\left\langle\nabla_{l}\left(\nabla_{k} \nabla h\right), \nabla h\right\rangle+2\left\langle\nabla_{k} \nabla h, \nabla_{l} \nabla h\right\rangle-h_{k l} d_{\omega}|\nabla h|^{2} \\
& =2\left\langle\nabla_{k, l}^{2}(\nabla h), \nabla h\right\rangle+2\left\langle h_{k l} \nabla_{\omega} \nabla h, \nabla h\right\rangle+2\left\langle\nabla_{k} \nabla h, \nabla_{l} \nabla h\right\rangle-h_{k l} d_{\omega}|\nabla h|^{2} \\
& =2\left\langle\nabla_{k, l}^{2}(\nabla h), \nabla h\right\rangle+2\left\langle\nabla_{k} \nabla h, \nabla_{l} \nabla h\right\rangle,
\end{aligned}
$$

after cancellation. Taking traces we find

$$
\left(\partial_{t}-\Delta\right)|\nabla h|^{2}=-2\left|\nabla^{2} h\right|^{2}+2\left\langle\left(\partial_{t}-\Delta\right)(\nabla h), \nabla h\right\rangle+3\left(\partial_{t} g^{i j}\right)\left\langle\nabla_{i} h, \nabla_{j} h\right\rangle .
$$

Commutation of covariant derivatives introduces the Riemann curvature tensor, and the time derivative of the connection is also needed:

$$
\left(\partial_{t}-\Delta\right)(\nabla h)=\nabla\left[\left(\partial_{t}-\Delta\right) h\right]+(\nabla \mathrm{Rm}) * h+\mathrm{Rm} *(\nabla h)+\left(\partial_{t} \Gamma\right) * h,
$$

where (see appendix)

$$
\partial_{t} h=\nabla d H+H \nabla_{\omega} h+T+h^{(3)}, \quad T_{i j}=H_{i} h\left(\omega, \partial_{j}\right)+H_{j} h\left(\omega, \partial_{i}\right),
$$

which combined with $\Gamma=h \omega$ and $\partial_{t} \omega=\nabla H+h^{(2)}$ is easily seen to imply

$$
\partial_{t} \Gamma=(\nabla d H) \omega+\nabla h * h+h^{(3)} \sim \nabla^{2} h+\nabla h * h+h^{(3)} .
$$

From the Gauss equation, $\mathrm{Rm} \sim h * h$. Thus

$$
\left\langle\left(\partial_{t}-\Delta\right)(\nabla h), \nabla h\right\rangle \sim\left\langle\nabla\left[\left(\partial_{t}-\Delta\right) h\right], \nabla h\right\rangle+\nabla^{2} h * \nabla h * h+(\nabla h)^{(2)} * h^{(2)}+\nabla h * h^{(4)} .
$$

On the other hand, from the evolution equation for $h$ (Appendix B) we have

$$
\left\langle\nabla\left[\left(\partial_{t}-\Delta\right) h\right], \nabla h\right\rangle=\left\langle\nabla\left(H \nabla_{\omega} h+T+h^{(3)}\right), \nabla h\right\rangle=\left\langle\nabla\left(H \nabla_{\omega} h\right), \nabla h\right\rangle+\langle\nabla T, \nabla h\rangle+(\nabla h)^{(2)} * h^{(2)}
$$


Computing the terms on the right, we find

$$
\left\langle\nabla\left(H \nabla_{\omega} h\right), \nabla h\right\rangle=\left\langle\nabla_{\omega} h, \nabla_{\nabla H} h\right\rangle+H\left\langle\nabla\left(\nabla_{\omega} h\right), \nabla h\right\rangle=\left\langle\nabla_{\omega} h, \nabla_{\nabla H} h\right\rangle+\nabla^{2} h * \nabla h * h,
$$

and using the Codazzi identity

$$
\langle\nabla T, \nabla h\rangle=2\left\langle\nabla_{\omega} h, \nabla_{\nabla H} h\right\rangle+\nabla^{2} h * \nabla h * h+(\nabla h)^{(2)} * h^{(2)} .
$$

Putting together these results, we have

$$
\left\langle\left(\partial_{t}-\Delta\right)(\nabla h), \nabla h\right\rangle=3\left\langle\nabla_{\omega} h, \nabla_{\nabla H} h\right\rangle+\nabla^{2} h * \nabla h * h+(\nabla h)^{(2)} * h^{(2)}+\nabla h * h^{(4)} .
$$

On the other hand, using the expression for $\partial_{t} g^{i j}$ given in the appendix we find

$$
3 \partial_{t} g^{i j}\left\langle\nabla_{i} h, \nabla_{j} h\right\rangle=-6\left\langle\nabla_{\omega} h, \nabla_{\nabla H} h\right\rangle+(\nabla h)^{(2)} * h^{(2)} .
$$

So we have cancellation, and obtain the evolution equation

$$
\left(\partial_{t}-\Delta\right)|\nabla h|^{2}=-2\left|\nabla^{2} h\right|^{2}+\nabla^{2} h * \nabla h * h+(\nabla h)^{(2)} * h^{(2)}+\nabla h * h^{(4)} .
$$

Remark. Without the cancellation, the right-hand side would involve terms of type $(\nabla h)^{(3)}$, which would be a problem for the argument that follows.

Given this calculation, the following lemma has a very simple proof.

Lemma 13.2. For a solution $w \in C^{5,3}(E)$, assume we have a uniform bound for $h:|h| \leq a_{0}$ in $E$. Then there are constants $\alpha>0, C>0$ depending only on the dimension and $a_{0}$, so that the function

$$
f(x, t)=\alpha|\nabla h|^{2}+|h|^{2}
$$

is a subsolution in $E$, that is, $\left(\partial_{t}-\Delta\right) f \leq C$.

Proof. The calculation above implies that

$$
\left(\partial_{t}-\Delta\right)|\nabla h|^{2} \leq-2\left|\nabla^{2} h\right|^{2}+c_{n}\left(a_{0}\left|\nabla^{2} h\right||\nabla h|+a_{0}^{2}|\nabla h|^{2}+a_{0}^{4}|\nabla h|\right),
$$

while the evolution equation for $|h|^{2}$ implies that

$$
\left(\partial_{t}-\Delta h\right)|h|^{2} \leq-2|\nabla h|^{2}+c_{n}\left(a_{0}^{2}|\nabla h|+a_{0}^{4}\right) .
$$

Clearly we may choose $\alpha$ small enough to satisfy the claim.

Our next goal is to extend this argument to higher covariant derivatives of $h$. It turns out this does not involve a cancellation similar to the one noted above. The terms appearing in each expression below all have the same weight, the weight of a term $T=\left(\nabla^{j_{1}} h\right)^{\left(p_{1}\right)} * \cdots *\left(\nabla^{j_{r}} h\right)^{\left(p_{r}\right)}$ being the positive integer

$$
w[T]=\sum_{i=1}^{r} p_{i}\left(j_{i}+1\right)
$$

—in particular, $w\left[\nabla^{j} h\right]=j+1$ and $w\left[\left(\nabla^{j} h\right)^{(p)}\right]=p(j+1)$ for $j \geq 0$, and $p \geq 1$. We introduce a convenient notation for the "error terms". For integers $w_{0} \geq 1$ and $n \geq 0$, the notation $\tilde{E}^{w_{0}, n}$ is used for a generic term of weight $w_{0}$ and involving covariant derivatives of $h$ of order at most $n$; i.e.,

$$
T=\tilde{E}^{w_{0}, n} \quad \text { means } \quad w[T]=w_{0}, \quad j_{i} \leq n .
$$


The symbol $E^{w_{0}, n}$ denotes such a term satisfying the additional restrictions

$$
p_{i}= \begin{cases}1 & \text { if } j_{i}=n \\ 1 \text { or } 2 & \text { if } j_{i}=n-1 \text { and } n \geq 1 .\end{cases}
$$

Sometimes the same notation is used for the real vector space spanned by terms of the given type. For example, above we showed that

$$
\left(\partial_{t}-\Delta\right)|h|^{2}=-2|\nabla h|^{2}+E^{4,1} \quad \text { and } \quad\left(\partial_{t}-\Delta\right)|\nabla h|^{2}=-2\left|\nabla^{2} h\right|^{2}+E^{6,2} .
$$

These symbols have some useful properties. For example, one sees by induction that

$$
\nabla\left(E^{n+3, n+1}\right) \subset E^{n+4, n+2}, \quad n \geq 0,
$$

using the easily checked fact that

$$
E^{n+3, n+1}=\left(\nabla^{n+1} h\right) * h+\left(\nabla^{n} h\right) *\left[\nabla h+h^{(2)}\right]+\tilde{E}^{n+3, n-1}, \quad n>1 .
$$

The property (13-1) generalizes to higher $n$ :

Lemma 13.3.

$$
\left(\partial_{t}-\Delta\right)\left|\nabla^{n} h\right|^{2}=-2\left|\nabla^{n+1} h\right|^{2}+E^{2 n+4, n+1} \quad \text { for } n \geq 0 .
$$

Proof ( for $n \geq 2$ ). With the natural multiindex notation,

$$
\partial_{t}|\nabla h|^{2}=2\left\langle\partial_{t}\left(\nabla^{n} h\right), \nabla^{n} h\right\rangle+\partial_{t}\left(g^{I J} g^{p r} g^{q s}\right)\left(\nabla_{I}^{n} h\right)_{p q}\left(\nabla_{J}^{n} h\right)_{r s}, \quad|I|=|J|=n .
$$

Using the Codazzi identity and the curvature tensor repeatedly, we obtain

$$
\begin{aligned}
\partial_{t}\left(g^{I J} g^{p r} g^{q s}\right)\left(\nabla_{I}^{n} h\right)_{p q}\left(\nabla_{J}^{n} h\right)_{r s}= & (n+2)\left(\partial_{t} g^{i j}\right)\left\langle\nabla_{i} \nabla^{n-1} h, \nabla_{j} \nabla^{n-1} h\right\rangle \\
& +\left(\partial_{t} g^{i j}\right) \operatorname{Rm}\left[\nabla^{n-2} h\right]_{i} * \operatorname{Rm}\left[\nabla^{n-2} h\right]_{j}+\left(\partial_{t} g^{i j}\right)\left(\nabla^{n} h\right)_{i} * \operatorname{Rm}\left[\nabla^{n-2} h\right]_{j} .
\end{aligned}
$$

Since $\partial_{t} g^{i j}=\nabla h+h^{(2)}$ (see (B-4) in Appendix B) and $\mathrm{Rm}=h * h$, this reduces to

$$
\left(\nabla^{n} h\right)^{(2)} *\left(\nabla h+h^{(2)}\right)+\left(\nabla^{n-2} h\right)^{(2)} *\left(\nabla h+h^{(2)}\right) * h^{(4)}+\left(\nabla^{n} h\right) *\left(\nabla^{n-2} h\right) *\left(\nabla h+h^{(2)}\right) * h^{(2)},
$$

which is in $E^{2 n+4, n+1}$.

Turning to space derivatives, we have (as for $n=1$ )

$$
\Delta\left|\nabla^{n} h\right|^{2}=2\left\langle\Delta\left(\nabla^{n} h\right), \nabla^{n} h\right\rangle+2\left|\nabla^{n+1} h\right|^{2},
$$

and therefore

$$
\left(\partial_{t}-\Delta\right)\left|\nabla^{n} h\right|^{2}=-2\left|\nabla^{n+1} h\right|^{2}+2\left\langle\left(\partial_{t}-\Delta\right)\left(\nabla^{n} h\right), \nabla^{n} h\right\rangle+E^{2 n+4, n+1} .
$$

The conclusion of the lemma is now an immediate consequence of the next claim, and of the expression (13-2) for a general term in $E^{n+3, n+1}$.

Claim.

$$
\left(\partial_{t}-\Delta\right)\left[\nabla^{n} h\right] \in E^{n+3, n+1} \text { for } n \geq 0 .
$$

Proof. We work by induction on $n$, the cases $n=0,1$ having already been checked:

$$
\left(\partial_{t}-\Delta\right) h=H \nabla_{\omega} h+T+h^{(3)} \in E^{3,1}, \quad\left(\partial_{t}-\Delta\right)(\nabla h)=\nabla\left[\left(\partial_{t}-\Delta\right) h\right]+E^{4,2} \in E^{4,2} .
$$


For the induction step, it is enough to show that

$$
\left(\partial_{t}-\Delta\right)\left[\nabla^{n+1} h\right]=\nabla\left[\left(\partial_{t}-\Delta\right)\left(\nabla^{n} h\right)\right]+E^{n+4, n+2},
$$

since $\nabla E^{n+3, n+1} \subset E^{n+4, n+2}$.

For the time derivative part, we have, for any multiindex $i I$ of length $n+1$ (with $n=|I|$ ),

$$
\begin{aligned}
\partial_{t}\left[\nabla^{n+1} h\right]_{i I} & =\partial_{t}\left[\partial_{i}\left(\nabla^{n} h\left[\partial_{I}\right]\right)-\left(\nabla^{n} h\right)\left(\nabla_{i} \partial_{I}\right)\right]=\partial_{i}\left(\partial_{t}\left(\nabla^{n} h\left[\partial_{I}\right]\right)\right)-\partial_{t}\left(\nabla^{n} h\left[\nabla_{i} \partial_{I}\right]\right) \\
& =\nabla_{i}\left(\partial_{t}\left(\nabla^{n} h\right)\right)\left[\partial_{I}\right]+\partial_{t}\left(\nabla^{n} h\right)\left(\nabla_{i} \partial_{I}\right)-\partial_{t}\left(\nabla^{n} h\right)\left(\nabla_{i} \partial_{I}\right)-\nabla^{n} h\left[\partial_{t}\left(\nabla_{i} \partial_{I}\right)\right] .
\end{aligned}
$$

For a multiindex $I=i_{1} \ldots i_{n}$ of length $n$ denote by $I_{p}^{k}$ the multiindex of length $n$ obtained from $I$ by setting its $k$-th entry $i_{k}$ equal to $p$. It is then clear that

$$
\partial_{t}\left(\nabla_{i} \partial_{I}\right)=\sum_{k=1}^{n} \sum_{p}\left(\partial_{t} \Gamma_{i i_{k}}^{p}\right) \partial_{I_{p}^{k}}
$$

In symbolic notation, the preceding calculation is summarized as

$$
\partial_{t}\left[\nabla^{n+1} h\right]=\nabla\left(\partial_{t} \nabla^{n} h\right)+\left(\nabla^{n} h\right) *\left(\partial_{t} \Gamma\right) .
$$

Since $\partial_{t} \Gamma \in E^{3,2}$, this says

$$
\partial_{t}\left[\nabla^{n+1} h\right]=\nabla\left(\partial_{t} \nabla^{n} h\right)+E^{n+4, n+2} .
$$

Covariant derivatives in space may be dealt with in the usual way. Again for a multiindex $i I$ of length $n+1$, we have for first-order derivatives

$$
\begin{aligned}
\nabla_{k}\left(\nabla_{i I}^{n+1} h\right) & =\nabla_{k}\left(\nabla_{i}\left(\nabla_{I}^{n} h\right)\right)-\nabla_{k}\left(\nabla^{n} h\left(\nabla_{i} \partial_{I}\right)\right) \\
& =\nabla_{i}\left(\nabla_{k}\left(\nabla_{I}^{n} h\right)\right)-\nabla_{k}\left(\nabla^{n} h\left(\nabla_{i} \partial_{I}\right)\right)+\operatorname{Rm}_{i k}\left[\nabla_{I}^{n} h\right]
\end{aligned}
$$

and for second-order covariant derivatives

$$
\begin{aligned}
\begin{aligned}
\nabla_{l}\left(\nabla_{k}\left(\nabla_{i I}^{n+1} h\right)\right) & =\nabla_{l}\left(\nabla_{i}\left(\nabla_{k}\left(\nabla_{I}^{n} h\right)\right)\right)+\nabla_{l}\left(\operatorname{Rm}_{i k}\left[\nabla_{I}^{n} h\right]\right)-\nabla_{l}\left(\nabla_{k}\left(\nabla^{n} h\left(\nabla_{i} \partial_{I}\right)\right)\right) \\
& =\nabla_{i}\left(\nabla_{l}\left(\nabla_{k}\left(\nabla_{I}^{n} h\right)\right)\right)+\operatorname{Rm}_{i l}\left[\nabla_{k}\left(\nabla_{I}^{n} h\right)\right]+\nabla\left(\operatorname{Rm} * \nabla^{n} h\right)+\nabla^{2}\left(\nabla^{n} h * h\right) \\
& =\nabla_{i}\left(\nabla_{l, k}^{2}\left(\nabla_{I}^{n} h\right)\right)+\nabla_{i}\left(\nabla_{\nabla_{l} \partial_{k}} \nabla_{I}^{n} h\right)+\operatorname{Rm} * \nabla^{n+1} h+\nabla\left(\operatorname{Rm} * \nabla^{n} h\right)+\nabla^{2}\left(\nabla^{n} h * h\right)
\end{aligned} \\
\begin{array}{r}
\nabla_{l, k}^{2}\left(\nabla_{i I}^{n+1} h\right)= \\
\nabla_{i}\left(\nabla_{l, k}^{2}\left(\nabla_{I}^{n} h\right)\right)-\nabla_{\nabla_{l} \partial_{k}}\left(\nabla_{i I}^{n+1} h\right)+\nabla\left(\Gamma * \nabla^{n+1} h\right) \\
\quad+\operatorname{Rm} * \nabla^{n+1} h+\nabla\left(\operatorname{Rm} * \nabla^{n} h\right)+\nabla^{2}\left(\nabla^{n} h * h\right) \\
=\nabla_{i}\left(\nabla_{l, k}^{2}\left(\nabla_{I}^{n} h\right)\right)+\Gamma * \nabla^{n+2} h+\nabla\left(\Gamma * \nabla^{n+1} h\right)+\operatorname{Rm} * \nabla^{n+1} h+\nabla\left(\operatorname{Rm} * \nabla^{n} h\right)+\nabla^{2}\left(\nabla^{n} h * h\right) .
\end{array}
\end{aligned}
$$

Taking traces with $g^{k l}$ and using the expressions $\mathrm{Rm}=h^{(2)}, \nabla \mathrm{Rm}=\nabla h * h, \Gamma=h \omega, \nabla \Gamma=\nabla h+h^{(2)}$, it follows easily that

$$
\Delta\left(\nabla^{n+1} h\right)=\nabla\left(\Delta\left(\nabla^{n} h\right)\right)+E^{n+4, n+2}
$$

and therefore

$$
\left(\partial_{t}-\Delta\right)\left[\nabla^{n+1} h\right]=\nabla\left[\left(\partial_{t}-\Delta\right)\left(\nabla^{n} h\right)\right]+E^{n+4, n+2},
$$

proving the claim and the lemma.

The analog of Lemma 13.2 for higher covariant derivatives of $h$ follows easily from these remarks. 
Lemma 13.4. For a solution $w \in C^{n+5,[(n+5) / 2]+1}(E)$ assume we have a uniform bound for $h$ and its first $n$ covariant derivatives $\left|\nabla^{j} h\right| \leq a_{j}$ in $E, j=0, \ldots, n$. Then there are constants $\alpha>0, C>0$ depending only on the dimension and the $a_{j}$, so that the function

$$
f_{n+1}(x, t)=\alpha\left|\nabla^{n+1} h\right|^{2}+\left|\nabla^{n} h\right|^{2}
$$

is a subsolution in $E$, that is, $\left(\partial_{t}-\Delta\right) f_{n+1} \leq C$.

Proof. In the proof we denote by $C_{n}$ a generic positive constant depending only on dimension and the $a_{j}, j=0, \ldots, n$. We have

$$
\left(\partial_{t}-\Delta\right)\left|\nabla^{n} h\right|^{2}=-2\left|\nabla^{n+1} h\right|^{2}+E^{2 n+4, n+1}, \quad\left(\partial_{t}-\Delta\right)\left|\nabla^{n+1} h\right|^{2}=-2\left|\nabla^{n+1} h\right|^{2}+E^{2 n+6, n+2},
$$

where

$$
\begin{aligned}
& E^{2 n+4, n+1}=\nabla^{n+1} h * \nabla^{n} h * h+\left(\nabla^{n} h\right)^{(2)} * \tilde{E}^{2,1}+\left(\nabla^{n} h\right) * \tilde{E}^{n+3, n-1}+\tilde{E}^{2 n+4, n-1}, \\
& E^{2 n+6, n+2}=\nabla^{n+2} h * \nabla^{n+1} h * h+\left(\nabla^{n+1} h\right)^{(2)} * \tilde{E}^{2,1}+\left(\nabla^{n+1} h\right) * \tilde{E}^{n+4, n}+\tilde{E}^{2 n+6, n} .
\end{aligned}
$$

This implies

$$
\begin{aligned}
\left(\partial_{t}-\Delta\right)\left|\nabla^{n} h\right|^{2} & \leq-2\left|\nabla^{n+1} h\right|^{2}+C_{n}\left|\nabla^{n+1} h\right|+C_{n}, \\
\left(\partial_{t}-\Delta\right)\left|\nabla^{n+1} h\right|^{2} & \leq-2\left|\nabla^{n+2} h\right|^{2}+C_{n}\left|\nabla^{n+2} h\right|\left|\nabla^{n+1} h\right|+C_{n}\left(\left|\nabla^{n+1} h\right|^{2}+\left|\nabla^{n+1} h\right|+1\right) .
\end{aligned}
$$

It is easy to see from these inequalities that $\alpha$ can be chosen sufficiently small so that the conclusion of the lemma will hold.

\section{Hölder gradient estimate for the second fundamental form}

Notation. In this section, parabolic Hölder spaces are denoted by a single superscript; i.e., $C^{2+\alpha,(1+\alpha) / 2}$ becomes $C^{2+\alpha}$, etc. Capital $X, Y$, etc., denote general points in the spacetime domain $E$. This follows the notation used in [Lieberman 1996].

A continuation criterion for the solution $w(y, t)$ in $E^{T}$ in terms of a bound on the norm $|h|_{g}$ of the second fundamental form would follow from an a priori $C^{3+\delta}\left(E^{T}\right)$ bound on a solution, assuming $|h|_{g} \leq a_{0}$ in $\bar{E}^{T}$; equivalently, from a global a priori Hölder gradient bound $|\nabla h|_{\delta} \leq M$ in $\bar{E}^{T}$ (for suitably controlled $M$ ). In this section we show how such a bound follows from the a priori estimates of linear parabolic theory applied to the evolution equations for $v, H$, and the Weingarten operator, under an additional hypothesis.

Assuming $w \in C^{2+\delta}\left(E^{T}\right)$ is a solution, satisfying in addition $|h|_{g} \leq a_{0}$ in $E^{T}$, we already observed the maximum principle implies bounds

$$
0 \leq w \leq w_{0}, \quad 1 \leq v \leq \bar{v} \text { in } \bar{E}^{T},
$$

depending only on the initial data and $\beta$ (we assume $w \geq 0$, at $t=0$, vanishing only on $\partial D_{0}$.) In particular, $g$ is uniformly equivalent to the euclidean metric on $E^{T}$. In this section, bounds depending on $a_{0}, \bar{v}$, and the initial data will be denoted generically by a constant $M>0$ (dependence on $\beta$ will not be 
recorded explicitly). The bound on $h$ implies a uniform $C^{2}$ bound for the spacetime domain $E^{T}$, which we can express in terms of a diffeomorphism $\Phi: D_{0} \times[0, T] \rightarrow E^{T}$ by

$$
|\Phi|_{C^{2}} \leq M
$$

We will also need to assume a uniform gradient bound on the boundary for the second fundamental form:

$$
\left|\left(\nabla_{\tau} h\right)(\tau, \tau)\right| \leq a_{1} \quad \text { for all } \tau \in T \partial D(t) \text { with }|\tau|=1
$$

Estimates depending $a_{0}, a_{1}, \bar{v}$ and the initial data will be given in terms of constants denoted generically by $M_{1}$.

In fact $E^{T}$ is a bounded domain in $\mathbb{R}^{n} \times[0, T]$ of class $C^{2+\delta}$ with bounds controlled by $M_{1}$. (This statement includes some regularity in $t$, so it is not immediate from the uniform bound assumed for $\nabla^{\tan } h^{\tan }$ on $\partial_{l} E$ ). To see this, consider the equation satisfied by $w_{k}=\partial_{k} w$, written in "divergence form" with Dirichlet boundary conditions

$$
\left\{\begin{array}{l}
\partial_{t} w_{k}-\partial_{i}\left(g^{i j} \partial_{j} w_{k}\right)=g^{k}:=\left(\partial_{k} g^{i j}\right) w_{i j}-\left(\partial_{i} g^{i j}\right) \partial_{j} w_{k} \\
w_{k \mid \partial_{l} E}:=\varphi^{k}=\left(\beta_{0} / \beta\right) n^{k}, \quad w_{k \mid t=0}=\partial_{k} w_{0} .
\end{array}\right.
$$

Assuming $\partial_{k} w \in C^{1+\delta}(E)$, the following estimate holds [Lieberman 1996, Theorem 4.27]:

$$
\left|w_{k}\right|_{1+\delta} \leq C\left(\sup _{E}\left|w_{k}\right|+\left\|g^{k}\right\|_{1, n+1+\delta}+\left|\varphi^{k}\right|_{1+\delta ; \partial_{l} E}+\left|\partial_{k} w_{0}\right|_{1+\delta ; D_{0}}\right) .
$$

Here $\left\|g^{k}\right\|_{1, n+1+\delta}$ is the norm in the spacetime Morrey space $L^{1, n+1+\delta}(E)$

$$
\left\|g^{k}\right\|_{1, n+1+\delta}=\sup _{\substack{Y \in E, r<\operatorname{diam} E}}\left(r^{-(n+1+\delta)} \int_{E[Y, r]}\left|g^{n}\right| d X\right) .
$$

In the present case this can easily be estimated, since

$$
\left|\partial_{k} g^{i j}\right|=\left|h_{k}^{i} \omega^{j}+h_{k}^{j} \omega^{i}\right| \leq M, \quad\left|\partial_{j} w_{k}\right| \leq \bar{v} a_{0} \leq M \quad \Longrightarrow \quad\left|g^{k}\right| \leq M
$$

and $|E[Y, r]| \leq C r^{n+2}$, while $\delta \in(0,1)$. Thus $\left\|g^{k}\right\|_{1, n+1+\delta} \leq M$.

Since $\left|\nabla_{\tau}\left(\nabla_{\tau} n\right)\right| \leq c\left(\left|\left(\nabla_{\tau} h\right)(\tau, \tau)\right|+|h|\right) \leq M_{1}$, it follows that $n$ is $C^{2}$ in space variables on $\partial_{l} E$. On the other hand, $D w=\omega / \beta$ on $\partial D(t)$, and $\omega$ is a solution of $\partial_{t} \omega^{k}=\operatorname{tr}_{g} D^{2} \omega^{k}+|h|_{g}^{2} \omega^{k}$, hence $n$ is also $C^{1}$ in time on $\partial_{l} E$. We conclude $\left|\varphi^{k}\right|_{1+\delta ; \partial_{l} E} \leq\left(\beta_{0} / \beta\right)|n|_{1+\delta ; \partial_{l} E} \leq M_{1}$.

Therefore we have $|D w|_{1+\delta} \leq M_{1}$, and $|w|_{2+\delta} \leq M_{1}$ (note that $C$ depends on $\left|g^{i j}\right|_{C^{\delta}}$ and other constants also controlled by $M$.) In particular, $E^{T}$ is a $C^{2+\delta}$ domain with chart constants controlled by $M_{1}$. (In fact, in a neighborhood of any point $P \in \partial_{l} E$ with $\partial_{y_{2}} w \neq 0$, a boundary chart $\Psi$ is given by $\Psi\left(y_{1}, y_{2}, t\right)=\left(y_{1}, w(y, t), t\right)$.)

The first-order term in the evolution equation for $h$ (or for the Weingarten operator) involves $D H$; hence the next step is to obtain a global gradient bound $|D H|_{1+\alpha} \leq M_{1}$ in $\bar{E}^{T}$. The mean curvature satisfies the "divergence form" equation with Neumann boundary conditions

$$
\left\{\begin{array}{l}
\partial_{t} H-\partial_{j}\left(g^{i j}(X) H_{i}\right)+\partial_{j}\left(g^{i j}\right)(X) \partial_{i} H-c(X) H=0, \\
d_{n} H=\left(\beta^{2} / \beta_{0}\right) H h_{n n}:=\psi \text { on } \partial D(t), \quad H_{\mid t=0}=H_{0},
\end{array}\right.
$$


where

$$
c:=|h|_{g}^{2}-h^{2}(\omega, \omega)+H h(\omega, \omega) .
$$

Then with the regularity conditions for the domain and the coefficients

$$
\partial_{l} E \in C^{1+\delta}, \quad n \in C^{\delta}\left(\partial_{l} E\right), \quad \partial_{j}\left(g^{i j}\right) \in L^{1, n+1+\delta}(E), \quad c \in L^{1, n+1+\delta}(E),
$$

and assuming $H \in C^{1+\delta}(E)$, or $w \in C^{3+\delta}(E)$, we have the bound

$$
|H|_{1+\delta ; \bar{E}} \leq C\left(\sup _{E}|H|+|\psi|_{\delta ; \partial_{l} E}+\left|H_{0}\right|_{1+\delta ; D_{0}}\right) .
$$

As noted earlier

$$
\left\|\partial_{j} g^{i j}\right\|_{1, n+1+\delta}+\|c\|_{1, n+1+\delta} \leq M,
$$

hence $C$ is controlled by $M$. In addition, $|w|_{2+\delta} \leq M_{1}$ implies $|h|_{\delta} \leq M_{1}$, and hence $|\psi|_{\delta ; \partial_{l} E} \leq M_{1}$. We conclude $|H|_{1+\delta} \leq M_{1}$, and state it as a lemma.

Lemma 14.1. Let $w \in C^{3+\delta}\left(E^{T}\right)$ be a classical solution of graph mean curvature motion with contact and constant-angle boundary conditions. Assume that $|h|_{g} \leq a_{0}$ on $\partial_{l} E$ and that $\left|\left(\nabla_{\tau} h\right)(\tau, \tau)\right| \leq a_{1}$ on $\partial_{l} E$. Then we have a global gradient bound for $H$ :

$$
\sup _{\bar{E}^{T}}|D H|_{\delta} \leq M_{1},
$$

for a constant $M_{1}$ depending on $\delta, \bar{v}, a_{0}, a_{1}$ and the initial data $w_{0}$.

Corollary 14.2. Under the same hypotheses as Lemma 14.1, we have a global gradient bound

$$
\sup _{\bar{E}}|\nabla h|_{g} \leq M_{1}
$$

for a positive constant $M_{1}$ depending on $\delta, \bar{v}, a_{0}, a_{1}$ and the initial data $w_{0}$.

Proof. The bound on the components $\left(\nabla_{n} h\right)(\tau, \tau)$ and $\left(\nabla_{n} h\right)(n, n)$ on the lateral boundary $\partial_{l} E$ follows immediately from the expressions in Section 11. The bound on $\left(\nabla_{\tau} h\right)(\tau, \tau)$ over $\partial_{l} E$ is hypothesized, and then the bound on the remaining component $\left(\nabla_{\tau} h\right)(n, n)$ follows from the global gradient bound $|D H| \leq M$ implied by Lemma 14.1. Thus $|\nabla h| \leq M_{1}$ on $\partial_{l} E$, and then the global bound follows from Lemma 13.2 and the maximum principle.

To improve the conclusion of Corollary 14.2 to a Hölder gradient bound, it is natural to consider the evolution equation for $h$ with the Neumann-type boundary conditions derived in Section 11. One is then faced with the problem that those boundary conditions do not control components such as $\left(\nabla_{\tau} h\right)(\tau, \tau)$ on $\partial_{t} E$. So as a preliminary step we consider the evolution equation for $v$, which has the advantage that the boundary values are constant. Written in linear form, we have

$$
\left\{\begin{array}{l}
\partial_{t} v-g^{i j}(X) v_{i j}+b^{i}(X) \partial_{i} v+c(X) v=0 \\
v_{\mid \partial_{t} E}=1 / \beta, \quad v_{\mid t=0}=v_{0}
\end{array}\right.
$$

where

$$
g^{i j}(X)=\delta_{i j}-\frac{w_{i} w_{j}}{1+|D w|^{2}}(X), \quad b^{i}(X)=\frac{2 g^{i j} w_{k} w_{k j}}{1+|D w|^{2}}(X), \quad c(X)=|h|_{g}^{2}(X)
$$


We clearly have $g^{i j} \in C^{\delta}$ ( since $D w \in C^{\delta}$ ), as well as $b^{i}, c \in C^{\delta}$ (since $h \in C^{\delta}$ ), and $\partial_{l} E \in C^{2+\delta}$ with bounds controlled by $M_{1}$ in all cases as observed earlier. Therefore assuming $v \in C^{2+\delta}$ (equivalently, $w \in C^{3+\delta}$ ) we have the bound

$$
|v|_{2+\delta ; \bar{E}} \leq C\left(\sup _{E} v+\frac{1}{\beta}\right)
$$

with $C$ controlled by $M_{1}$. Thus $\left|D^{2} v\right|_{\delta} \leq M_{1}$. Recalling $v^{-1} \partial_{i} v=h\left(\partial_{i}, \omega\right)$, this implies that

$$
\left|\left(\nabla_{\tau} h\right)(n, n)\right|_{\delta ; \partial_{l} E}=\left|\left(\nabla_{n} h\right)(\tau, n)\right|_{\delta ; \partial_{l} E} \leq M_{1} \quad \text { for all } \tau \in T \partial D(t) \text { and }|\tau|=1 \text {. }
$$

Since $H=\beta^{2} h_{n n}+h(\tau, \tau)$ on $\partial_{l} E$, it follows from Lemma 14.1 that we also have $\left|\left(\nabla_{\tau} h\right)(\tau, \tau)\right|_{\delta ; \partial_{l} E} \leq M_{1}$. For the remaining components of $\nabla h$, this bound follows directly from the boundary conditions

$$
\left|\left(\nabla_{n} h\right)(\tau, \tau)\right|_{\delta ; \partial_{l} E}+\left|\left(\nabla_{n} h\right)(n, n)\right|_{\delta ; \partial_{l} E} \leq M_{1} .
$$

Now consider the evolution of the components of the Weingarten operator, written in divergence form with Neumann boundary conditions

$$
\begin{cases}\partial_{t} h_{j}^{k}-\partial_{i}\left(g^{i l} \partial_{l} h_{j}^{k}\right)=f_{j}^{k} & \text { in } E^{T}, \quad f_{j}^{k}:=H_{j} h_{l}^{k} \omega^{l}-H_{l} h_{j}^{l} \omega^{k}+h_{j}^{(3) k}-\left(\partial_{i} g^{i l}\right)\left(\partial_{l} h_{j}^{k}\right), \\ d_{n}\left(h_{j}^{k}\right)=\varphi_{j}^{k} & \text { on } \partial_{l} E, \quad h_{j \mid t=0}^{k}=h_{j 0}^{k} .\end{cases}
$$

The same theorem quoted above gives the estimate (assuming $h_{j}^{k} \in C^{1+\delta}$ or $w \in C^{3+\delta}$ )

$$
\left|h_{j}^{k}\right|_{1+\delta ; \bar{E}} \leq C\left(\sup _{E}\left|h_{j}^{k}\right|+\left\|f_{j}^{k}\right\|_{1, n+1+\delta}+\left|\varphi_{j}^{k}\right|_{\delta ; \partial_{l} E}+\left|h_{0 j}^{k}\right|_{1+\delta ; D_{0}}\right) .
$$

Note that

$$
d_{n}\left(h_{j}^{k}\right)=g^{i k}\left(\nabla_{n} h\right)_{i j}=\beta^{2}\left(\nabla_{n} h\right)\left(n, \partial_{j}\right) n^{k}+\left(\nabla_{n} h\right)\left(\tau, \partial_{j}\right) \tau^{k} \text { on } \partial_{l} E .
$$

From this and the above discussion it follows that $\left|\varphi_{j}^{k}\right|_{\delta ; \partial_{l} E} \leq M_{1}$. The bound $\left\|f_{j}^{k}\right\|_{1, n+1+\delta} \leq M_{1}$ follows from Lemma 14.1 and Corollary 14.2. We conclude $\left|h_{j}^{k}\right|_{1+\delta ; \bar{E}} \leq M_{1}$. The $1+\delta$ estimate for $h_{j}^{k}$ clearly implies the following lemma:

Lemma 14.3. Let $w \in C^{3+\delta}\left(E^{T}\right)$ be a classical solution of graph mean curvature motion with contact and constant-angle boundary conditions. Assume that $|h|_{g} \leq a_{0}$ on $\partial_{l} E$ and that $\left|\left(\nabla_{\tau} h\right)(\tau, \tau)\right|_{\partial_{l} E} \leq a_{1}$. Then we have a global Hölder gradient bound for $h$ :

$$
|\nabla h|_{\delta ; \bar{E}^{T}} \leq M_{1}
$$

for a constant $M_{1}$ depending on $\delta, \bar{v}, a_{0}, a_{1}$ and the initial data $w_{0}$.

Remark. This is clearly equivalent to a global a priori $C^{3+\delta}$ bound for $w$ on $\bar{E}^{T},|w|_{3+\delta} \leq M_{1}$.

Lemma 14.3 is the main step in the derivation of a "continuation criterion" for this flow.

Proposition 14.4. Assume the maximal existence time $T_{\max }$ is finite. Then (for $n=2$, in the concave case)

$$
\limsup _{t \rightarrow T_{\max }} \sup _{\partial D(t)}\left(|h|_{g}+\left|\left(\nabla_{\tau} h\right)(\tau, \tau)\right|\right)=\infty
$$


Proof. For $w_{0} \in C^{3+\bar{\alpha}}\left(D_{0}\right)$ satisfying the contact angle condition (with $\bar{\alpha} \in(0,1)$ arbitrary) and $\alpha=\bar{\alpha}^{2}$, Theorem 8.1 yields a unique solution $F=[u, \varphi]$ of mcm with contact angle/orthogonality boundary conditions in a maximal time interval $\left[0, T_{\max }\right]$ with $F \in C^{2+\alpha}\left(Q_{0}^{T_{\max }}\right), Q_{0}^{T_{\max }}=Q \times\left[0, T_{\max }\right)$; this is also the unique solution in $F \in C^{2+\delta^{2}}\left(Q_{0}^{T_{\max }}\right)$, where $\delta=\alpha^{2}$. Then $w=u \circ \varphi^{-1} \in C^{2+\delta}\left(E^{T_{\max }}\right)$ is a solution of graph mcm, which for any $t_{0}>0$ is in $C^{3+\delta}\left(E_{t_{0}}^{T_{\max }}\right)$. By contradiction, assume $|h|_{g}+\left|\left(\nabla_{\tau} h\right)(\tau, \tau)\right|$ is bounded in $E_{t_{0}}^{T}$ for any $T<T_{\max }$ (with bound independent of $T$ ). Then Lemma 14.3 applies, giving an a priori bound $|\nabla h|_{\delta ; \bar{E}_{t_{0}}} \leq M_{1}$, for $T$ arbitrarily close to $T_{\max }$. In particular, $|w(\cdot, T)|_{C^{3+\delta}(D(T))} \leq M_{1}$, and for $T$ close enough to $T_{\max }$ we can use Theorem 8.1 again, with initial data $w(\cdot, T)$, to find a solution $F^{\prime}=\left[u^{\prime}, \varphi^{\prime}\right] \in C^{2+\delta^{2}}\left(Q_{0}^{T^{\prime}}\right)$ (where $\left.T^{\prime}>T_{\max }\right)$, extending $F$. This contradicts the maximality of $T_{\max }$.

\section{Behavior at the extinction time}

In this section we consider the behavior of $\Sigma_{t}$ as $t$ approaches the maximal existence time $T$, in the concave case. We assume $H \leq H_{0}<0$ at $t=0$, so $T$ is finite. Let $K_{t} \subset \mathbb{R}^{n+1}$ be the compact convex set bounded by $\Sigma_{t}$. Since $H \leq 0,\left\{K_{t}\right\}$ is a decreasing family, and the intersection

$$
K_{T}=\bigcap_{0 \leq t<T} K_{t} \subset \mathbb{R}^{n+1}
$$

is compact, convex and nonempty. It turns out that $K_{T}$ has zero $(n+1)$-volume. In this section we use the support function to show this when $n=2$ (following the argument in [Stahl 1996]), under the assumption that there is no gradient blowup.

Assume the origin $0 \in \mathbb{R}^{n}$ is a point of $K_{T}$. The support function of $K_{t}$ (with respect to this origin) is the function $p(\cdot, t)$ on $D(t)$ given by

$$
p(y, t)=\langle G(y, t), N(y, t)\rangle, \quad G(y, t)=[y, w(y, t)] .
$$

Since $K_{t}$ is convex, $p>0$ in $D(t)$; the evolution equations and boundary conditions for $p$ are easily computed. From $L[G]=0$ and $L[N]=|h|_{g}^{2} N$, we have

$$
L[p]=\langle L[G], N\rangle+\langle G, L[N]\rangle-2 g^{k l}\left\langle\partial_{k} G, \partial_{l} N\right\rangle=|h|_{g}^{2} p+2 H,
$$

and, since $\left\langle d_{n} G, N\right\rangle=0$,

$$
p_{n \mid \partial D(t)}=\left\langle G, d_{n} N\right\rangle=-A\left(G^{T}, N\right),
$$

where, with $y^{T}:=y-(y \cdot n) n \in T_{y} \partial D(t)$, the tangential component $G^{T}:=G-\langle G, N\rangle N$ is easily seen to be, at $\partial D(t)$,

$$
G^{T}=\frac{1}{v^{2}}\left[w_{n}^{2} y^{T}+y, 0\right] .
$$

Since $h\left(y^{T}, n\right)=0$ at $\partial D(t)$, this implies $A\left(G^{T}, n\right)=\beta^{2}(y \cdot n) h(n, n)$. Note that $p(y)=-\beta_{0}(y \cdot n)$ on $\partial D(t)$, so we have

$$
p_{n \mid \partial D(t)}=\frac{\beta^{2}}{\beta_{0}} p h_{n n},
$$

which is reminiscent of the boundary condition for $H$. We also have the upper bound

$$
p \leq\|G\| \leq \max _{D(0)}\left\|G_{0}\right\|:=p_{0},
$$

since the $K_{t}$ are nested. 
Proposition 15.1. Let $n=2$. Assume that

$$
\limsup _{t \rightarrow T} \sup _{y \in \partial D(t)}|h|_{g}=\infty
$$

at the maximal existence time $T$. Then

$$
\liminf _{t \rightarrow T} \inf _{y \in D(t)} p(y, t)=0 .
$$

Proof. Reasoning by contradiction, assume $p>2 \delta>0$ for $t \in[0, T)$. We claim that this implies an upper bound for $|H|$ (and hence for $|h|$, since $|h|^{2} \leq n H^{2}$ ) contradicting the fact that $\lim _{\sup _{t \rightarrow T}} \sup _{\Gamma_{t}}|h|=\infty$.

To prove the claim, consider the function

$$
f(y, t):=\frac{|H|}{p-\delta}=-\frac{H}{p-\delta} .
$$

Using the evolution equations and boundary conditions for $H$ and $p$ we find (with $\hat{\omega}:=\omega /|\omega|_{g}$, see Remark 15.2 below)

and

$$
L[f]=f\left(-\delta|h|_{g}^{2}+2 p f\right)+|\omega|_{g}^{2}\left(h^{2}(\hat{\omega}, \hat{\omega})-H h(\hat{\omega}, \hat{\omega})\right)-\frac{2}{p-\delta} g^{k l} \partial_{k} f \partial_{l} p
$$

Since $|h|_{g}^{2} \geq \frac{1}{n} H^{2}=\frac{1}{n} f^{2}(p-\delta)^{2}$ we get

$$
f_{n \mid \partial D(t)}=-\delta \frac{\beta^{2}}{\beta_{0}} h_{n n} \frac{|h|^{2}}{(p-\delta)^{2}} \geq 0 .
$$

$$
L[f] \leq f\left(-\frac{(p-\delta)^{2} \delta}{n} f^{2}+2 p f\right)+|\omega|_{g}^{2}\left(h^{2}(\hat{\omega}, \hat{\omega})-H h(\hat{\omega}, \hat{\omega})\right)-\frac{2}{p-\delta}\langle\nabla f, \nabla p\rangle_{g} .
$$

Now recall from Remark 12.8 that if $n=2$

$$
h^{2}(\hat{\omega}, \hat{\omega})-H h(\hat{\omega}, \hat{\omega})=-\Delta \leq 0,
$$

so

$$
L[f] \leq f\left(-\frac{(p-\delta)^{2} \delta}{n} f^{2}+2 p f\right)-\frac{2}{p-\delta}\langle\nabla f, \nabla p\rangle_{g} .
$$

Let $\delta>0$ be so small that $\sup _{D(0)} f_{\mid t=0}<2 n p_{0} / \delta^{3}$. We claim this persists for all $t \in[0, T)$. If not, assume $f\left(y_{0}, t_{0}\right)=2 n p_{0} / \delta^{3}$ with $t_{0}>0$ smallest possible and let $y_{0}$ be a local maximum of $f\left(\cdot, t_{0}\right)$. Since $f_{n} \geq 0$ at $\partial D\left(t_{0}\right)$, the boundary point lemma implies that $z_{0}=\left(y_{0}, t_{0}\right)$ can't be a boundary point of $E$. Thus $y_{0} \in \partial D\left(t_{0}\right)$ is an interior point, so $L[f]_{z_{0}} \geq 0$ and $\nabla f\left(z_{0}\right)=0$ : hence

$$
\frac{(p-\delta)^{2} \delta}{n} f\left(z_{0}\right) \leq 2 p\left(z_{0}\right), \quad \text { or } \quad f\left(z_{0}\right) \leq \frac{2 n p\left(z_{0}\right)}{\delta(p-\delta)^{2}} \leq \frac{2 n p_{0}}{\delta(p-\delta)^{2}},
$$

which is not possible since $p-\delta>\delta$. Thus $f(y, t)<4 p_{0} / \delta^{3}$ in $E$, which implies the bound $|H| \leq 4 p_{0}^{2} / \delta^{3}$ for $t \in[0, T)$, contradicting the maximality of $T$.

Remark 15.2. It is easy to verify that the vector fields

$$
\omega=\frac{1}{v}\left[w_{1}, w_{2}\right], \quad \tilde{\omega}=v \omega^{\perp}=\left[-w_{2}, w_{1}\right]
$$


in $D(t) \subset \mathbb{R}^{2}$ satisfy

$$
\langle\omega, \tilde{\omega}\rangle_{g}=0, \quad|\omega|_{g}^{2}=|\tilde{\omega}|_{g}^{2}=|D w|_{e}^{2}:=w_{1}^{2}+w_{2}^{2} .
$$

Thus we may think of $\{\omega, \tilde{\omega}\}$ as a "conformal pseudoframe" ( $\omega$ and $\tilde{\omega}$ vanish when $D w=0)$, defined on all of $D(t)$. Moreover, at the boundary $\partial D(t)$,

$$
\omega=\beta_{0} n, \quad \tilde{\omega}=\frac{\beta_{0}}{\beta} n^{\perp}=\frac{\beta_{0}}{\beta} \tau,
$$

where $\{\tau, n\}$ is an euclidean-orthonormal frame along $\Gamma_{t}$. Thus $\omega$ and $\tilde{\omega}$ supply canonical extensions of $n, \tau$ to the interior of $D(t)$ as uniformly bounded vector fields.

It follows from the proposition that $K_{T}$ cannot contain a half-ball of positive radius centered at a point of $\mathbb{R}^{2}$; in particular, $\operatorname{vol}_{3}\left(K_{T}\right)=0$. Based on the experience with curve networks [Schnürer et al. 2007], one is led to expect that $K_{T}$ is a point $\left(\operatorname{diam} K_{T}=0\right)$, at least under the same assumption as the proposition (no gradient blowup). We have not been able to show this yet; existence of self-similar solutions and comparison arguments appropriate to the free-boundary setting appear to be needed for the usual approach to work.

\section{Final comments}

Local existence. We state here a local existence theorem for configurations of graphs over domains with moving boundaries. In this setting, a triple junction configuration consists of three embedded hypersurfaces $\Sigma^{1}, \Sigma^{2}, \Sigma^{3}$ in $\mathbb{R}^{n+1}$, graphs of functions $w^{I}$ defined over time-dependent domains $D^{1}(t), D^{2}(t) \subset$ $\mathbb{R}^{n}$ ( $D^{1}$ covered by one graph, $D^{2}$ by two graphs), satisfying the following conditions:

(1) The $\Sigma^{I}$ intersect along an $(n-1)$-dimensional graph $\Lambda(t)$ (the "junction"), along which the upward unit normals satisfy the relation: $N_{1}+N_{2}=N_{3}$.

(2) If a fixed support hypersurface $S \subset \mathbb{R}^{n+1}$ is given (also a graph, not necessarily connected), the $\Sigma^{I}$ intersect $S$ orthogonally.

Topologically, in the case of bounded domains one has the following examples:

(i) Lens type: two disks or two annuli covering $D^{2}(t)$ and one annulus covering $D^{1}(t)$.

(ii) Exterior type: two annuli covering $D^{2}(t)$ and one disk covering $D^{1}(t)$.

The boundary component of the annuli disjoint from the junction intersects the support hypersurface $S$ orthogonally for each $t$.

Let $\Sigma_{0}^{I}(I=1,2,3)$ be graphs of $C^{3+\alpha}$ functions over $C^{3+\alpha}$ domains $D_{0}^{1}, D_{0}^{2} \subset \mathbb{R}^{n}$, defining a triple junction configuration and satisfying the compatibility condition for the mean curvatures on the common boundary $\Gamma_{0}$ of $D_{0}^{1}$ and $D_{0}^{2}$

$$
H^{1}+H^{2}=H^{3} .
$$

Then there exists $T>0$ depending only on the initial data, and functions $w^{I} \in C^{2+\alpha, 1+\alpha / 2}\left(Q^{I}\right), Q^{I} \subset$ $\mathbb{R}^{n} \times[0, T)$, so that the graphs of $w^{I}(., t): D^{I}(t) \rightarrow \mathbb{R}$ define a triple junction configuration for each $t \in[0, T)$ moving by mean curvature.

The proof will be given elsewhere. 
Uniqueness. An interesting issue we have not addressed here is whether one has breakdown of uniqueness for initial data of lower regularity, or if the "orthogonality condition" at the junction is removed. For curve networks, nonuniqueness has been considered in [Mazzeo and Sáez 2007]; but neither a drop in regularity (from initial data to solution in Hölder spaces) nor the orthogonality condition play a role in the case of curves.

\section{Appendix A. Proof of Lemma 4.1}

Throughout the proof $n$ denotes the inner unit normal at $\partial D$, extended to a tubular neighborhood $\mathcal{N}$ in $\mathbb{R}^{n}$ so that $D_{n} n=0$. Since $D$ is uniformly $C^{3+\alpha}$, it follows that $n \in C^{2+\alpha}(\partial D)$ with uniform bounds. Denote by $\rho$ the oriented distance to the $\partial D$ (so $D \rho=n$ in $\mathcal{N}$ ). Let $\zeta \in C^{3}(\bar{D})$ be a cutoff function with $\zeta \equiv 1$ in $\mathcal{N}_{1} \subset \mathcal{N}, \zeta \equiv 0$ in $D \backslash \mathcal{N}$.

We find $\varphi$ of the form

$$
\varphi(x)=x+\zeta(x) f(x) n(x)
$$

with $f \in C^{2+\alpha}(\mathcal{N})$. The 1-jet conditions on $\varphi$ at $\partial D$ translate to these conditions on $f$ :

$$
f_{\mid \partial D}=0, \quad D f_{\mid \partial D}=0, \quad D^{2} f(n, n)_{\mid \partial D}=\Delta f_{\mid \partial D}=h .
$$

Lemma A.1. Let $D$ be a uniformly $C^{3+\alpha}$ domain with boundary distance function $\rho>0$. Let $h \in C^{\alpha}(\partial D)$ be bounded. There exists an extension $g \in C^{\infty}(D) \cap C(\bar{D})$ such that $g_{\mid \partial D}=h$, $\sup _{\bar{D}}|g| \leq \sup _{\partial D}|h|$ and $\rho^{2} g \in C^{2+\alpha}(\bar{D})$.

Given this lemma, all we have to do is set $f=\frac{1}{2} \rho^{2} g$, which clearly satisfies all the requirements (in particular, $\Delta f=h$ at $\partial D$.)

To verify that $\varphi$ is a diffeomorphism, it suffices to check that $|\zeta f n|_{C^{1}}$ (in $\mathcal{N} \subset\left\{\rho<\rho_{0}\right\}$ ) is small if $\rho_{0}$ is small. This is easily seen: $|\zeta f n|_{C^{0}} \leq \frac{1}{2} \rho_{0}^{2}|g|_{C^{0}}$; from $|D \zeta| \leq c \rho_{0}^{-1}$ it follows that $|f D \zeta| \leq c \rho_{0}|g|_{C^{0}}$; and $|D f| \leq \frac{1}{2} \rho_{0}^{\alpha}\|g\|_{C^{2+\alpha}(\bar{D})}$ on $\mathcal{N}$, since $D f \in C^{1+\alpha}(\bar{D})$ and $D f_{\mid \partial D}=0$. Finally, with $\mathscr{A}$ the second fundamental form of $\partial D$,

$$
|D n| \leq|\mathscr{A}|_{C^{0}} \Longrightarrow|f D n| \leq \frac{1}{2} \rho_{0}^{2}|g|_{C^{0}}|\mathscr{A}|_{C^{0}} .
$$

A word about Lemma A.1. (This is probably in the literature, but I don't know a reference.) If $D$ is the upper half-space, we solve $\Delta g=0$ in $D$ with boundary values $h$. Then the estimate

$$
\left[D^{2}\left(\rho^{2} P * h\right)\right]^{\alpha}(\bar{D}) \leq c|h|_{C^{\alpha}(\partial D)}
$$

follows by direct computation with the Poisson kernel $P$; for the rest of the norm, use interpolation. Then transfer the estimate to a general domain using "adapted local charts" in which $\rho$ in $D$ corresponds to the vertical coordinate in the upper half-space. (It is easy to see that at each boundary point there is a $C^{2+\alpha}$ adapted chart with uniform bounds.)

\section{Appendix B. Evolution equations for the second fundamental form}

We consider mean curvature motion of graphs:

$$
G(y, t)=[y, w(y, t)], \quad y \in D(t) \subset \mathbb{R}^{n}, \quad w_{t}=g^{i j} w_{i j}=v H, \quad v=\sqrt{1+|D w|^{2}} .
$$


In this appendix we include evolution equations for geometric quantities, in terms of the operators

$$
\partial_{t}-\Delta_{g}, \quad L=\partial_{t}-\operatorname{tr}_{g} d^{2} .
$$

It is often convenient to use the vector field in $D(t)$

$$
\omega:=\frac{1}{v} D w .
$$

Since $-\omega$ is the $\mathbb{R}^{n}$ component of the unit normal $N$ and $L[N]=|h|_{g}^{2} N$, we have

$$
L\left[\omega^{i}\right]=|h|_{g}^{2} \omega^{i}, \quad|h|_{g}^{2}:=g^{i k} g^{j l} h_{i j} h_{k l} .
$$

Here $h=\left(h_{i j}\right)$ is the pullback to $D(t)$ of the second fundamental form $A$ :

$$
h\left(\partial_{i}, \partial_{j}\right)=h_{i j}=A\left(G_{i}, G_{j}\right)=\frac{1}{v} w_{i j} .
$$

First, denoting by $\nabla$ the pullback to $D(t)$ of the induced connection $\nabla^{\Sigma}$ (that is, $G_{*}\left(\nabla_{X} Y\right)=\nabla_{G_{*} X}^{\Sigma} G_{*} Y$ for any vector fields $X, Y$ in $D(t)$ ), and using the definition

$$
\nabla_{G_{i}}^{\Sigma} G_{j}=G_{i j}-\left\langle G_{i j}, N\right\rangle N=\left[0, w_{i j}\right]-\frac{1}{v^{2}} w_{i j}[-D w, 1]=\frac{w_{i j}}{v^{2}}\left[D w,|D w|^{2}\right]=\frac{w_{i j}}{v^{2}} G_{*} D w,
$$

we conclude that

$$
\nabla_{\partial_{i}} \partial_{j}=\frac{1}{v} h_{i j} D w=h_{i j} \omega
$$

From this one derives easily a useful expression relating the Laplace-Beltrami operator and the operator $\operatorname{tr}_{g} d^{2}$ acting on functions

$$
\Delta_{g} f=\operatorname{tr}_{g} d^{2} f-\frac{H}{v} w_{m} f_{m}=\operatorname{tr}_{g} d^{2} f-H d_{\omega} f .
$$

We also have, for the covariant derivatives of $h$ with respect to the euclidean connection and to $\nabla=\nabla^{g}$ :

$$
\partial_{m}\left(h_{i j}\right)=\nabla_{m} h_{i j}+\left[h_{j m} h_{i k}+h_{i m} h_{j k}\right] \omega^{k} .
$$

(Here $\nabla h$ is the symmetric $(3,0)$-tensor with components: $\nabla_{m} h_{i j}=\left(\nabla_{\partial_{m}} h\right)\left(\partial_{i}, \partial_{j}\right)$.)

Iterating this and taking $g$-traces yields, using the Codazzi identity and the easily verified relation $\partial_{i} \omega^{k}=h_{i}^{k}:=g^{j k} h_{i j}$,

$$
\begin{aligned}
\operatorname{tr}_{g} d^{2}\left(h_{i j}\right)= & g^{m k} \partial_{m}\left(\partial_{k}\left(h_{i j}\right)\right) \\
= & g^{m k}\left(\nabla_{\partial_{m}, \partial_{k}}^{2} h\right)\left(\partial_{i}, \partial_{j}\right)+H \nabla_{\omega} h_{i j}+2\left[h_{i}^{k} \nabla_{k} h_{j p}+h_{j}^{k} \nabla_{k} h_{i p}\right] \omega^{p}+\left[H_{i} h_{j p}+H_{j} h_{i p}\right] \omega^{p} \\
& +2\left[h_{i p}\left(h^{2}\right)_{j q}+\left(h^{2}\right)_{i p} h_{j q}+H h_{i p} h_{j q}\right] \omega^{p} \omega^{q}+2\left(h^{3}\right)_{i j}+2\left(h^{2}\right)_{i j} h(\omega, \omega) .
\end{aligned}
$$

Here the powers $h^{2}$ and $h^{3}$ of $h$ are the symmetric 2-tensors defined used the metric:

$$
\left(h^{2}\right)_{i j}:=g^{k p} h_{i k} h_{p j}=h_{i}^{k} h_{p j}, \quad\left(h^{3}\right)_{i j}:=g^{k p} g^{l q} h_{i k} h_{p l} h_{q j} .
$$

Note also that

$$
\left[h_{i}^{k} \nabla_{k} h_{j p}+h_{j}^{k} \nabla_{k} h_{i p}\right] \omega^{p}=\nabla_{\omega}\left(h^{2}\right)_{i j}
$$

using the Codazzi identity. 
Evolution equations for $\boldsymbol{h}$. Starting from $G_{t}=v H e_{n+1}=H\left(N+v^{-1}\left[D w,|D w|^{2}\right]\right)=H N+H G_{*} \omega$ and $N_{t}=-\nabla^{\Sigma} H-H v^{-1} \nabla^{\Sigma} v$ (where $\nabla^{\Sigma} f=g^{i j} f_{j} G_{i}$ and $\nabla f=g^{i j} f_{j} \partial_{i}$ ) we have

$$
\partial_{t}\left(h_{i j}\right)=\left\langle(H N)_{i j}, N\right\rangle-\left\langle G_{i j}, \nabla^{\Sigma} H\right\rangle-\frac{H}{v}\left\langle G_{i j}, \nabla^{\Sigma} v\right\rangle+\left\langle\left(H G_{*} \omega\right)_{i j}, N\right\rangle .
$$

Using the easily derived facts

$$
\left\langle N_{i j}, N\right\rangle=-h^{2}\left(\partial_{i}, \partial_{j}\right), \quad H_{i j}-\left\langle G_{i j}, \nabla^{\Sigma} H\right\rangle=(\nabla d H)\left(\partial_{i}, \partial_{j}\right), \quad \frac{1}{v}\left\langle G_{i j}, \nabla^{\Sigma} v\right\rangle=h(\omega, \omega) h_{i j},
$$

we obtain

$$
\partial_{t}\left(h_{i j}\right)=(\nabla d H)\left(\partial_{i}, \partial_{j}\right)-H h^{2}\left(\partial_{i}, \partial_{j}\right)-H h(\omega, \omega) h_{i j}+\left\langle\left(H G_{*} \omega\right)_{i j}, N\right\rangle,
$$

where

$$
\left\langle\left(H G_{*} \omega\right)_{i j}, N\right\rangle=H_{i}\left\langle\left(G_{*} \omega\right)_{j}, N\right\rangle+H_{j}\left\langle\left(G_{*} \omega\right)_{i}, N\right\rangle+H\left\langle\left(G_{*} \omega\right)_{i j}, N\right\rangle .
$$

To identify the terms, computation shows that

$$
\left\langle\left(G_{*} \omega\right)_{i}, N\right\rangle=h\left(\omega, \partial_{i}\right),
$$

and hence, using also

$$
\nabla_{G_{i}}^{\Sigma}\left(G_{*} \omega\right)=G_{*}\left(\nabla_{\partial_{i}} \omega\right), \quad \nabla_{\partial_{i}} \omega=\left(h_{i}^{p}+\omega^{q} h_{i q} \omega^{p}\right) \partial_{p}=\sum_{p} h_{i p} \partial_{p},
$$

we obtain (using $\left.\omega^{k} \partial_{j}\left(h_{i k}\right)=\nabla_{\omega} h_{i j}+2 h\left(\partial_{i}, \omega\right) h\left(\partial_{j}, \omega\right)\right)$ that

$$
\begin{aligned}
\left\langle\left(G_{*} \omega\right)_{i j}, N\right\rangle & =\partial_{j}\left(\omega^{k} h_{i k}\right)-\left\langle\nabla_{G_{i}}^{\Sigma}\left(G_{*} \omega\right), \partial_{j} N\right\rangle=h_{j}^{k} h_{i k}+\omega^{k} \partial_{j}\left(h_{i k}\right)+h\left(\partial_{j}, \nabla_{\partial_{i}} \omega\right) \\
& =\left(\nabla_{\omega} h\right)_{i j}+\left(h^{2}\right)_{i j}+2 h\left(\omega, \partial_{i}\right) h\left(\omega, \partial_{j}\right)+\sum_{p} h_{i p} h_{j p} \\
& =\left(\nabla_{\omega} h\right)_{i j}+2\left(h^{2}\right)_{i j}+3 h\left(\omega, \partial_{i}\right) h\left(\omega, \partial_{j}\right),
\end{aligned}
$$

since $\sum_{p} h_{i p} h_{j p}=\left(h^{2}\right)_{i j}+h\left(\omega, \partial_{i}\right) h\left(\omega, \partial_{j}\right)$. Combining all the terms yields

$\partial_{t}\left(h_{i j}\right)=(\nabla d H)\left(\partial_{i}, \partial_{j}\right)+H \nabla_{\omega} h_{i j}+H_{i} h\left(\omega, \partial_{j}\right)+H_{j} h\left(\omega, \partial_{i}\right)$

$$
+H\left(h^{2}\right)_{i j}+3 H h\left(\omega, \partial_{i}\right) h\left(\omega, \partial_{j}\right)-H h(\omega, \omega) h_{i j} .
$$

From this expression and Simons' identity (in tensorial form)

$$
\nabla d H=\Delta_{g} h+|h|_{g}^{2} h-H h^{2},
$$

we obtain easily a tensorial "heat equation" for $h$ :

$$
\left[\left(\partial_{t}-\Delta_{g}\right) h\right]_{i j}=H \nabla_{\omega} h_{i j}+H_{i} h\left(\omega, \partial_{j}\right)+H_{j} h\left(\omega, \partial_{i}\right)+C_{i j},
$$

with

$$
C_{i j}:=|h|_{g}^{2} h_{i j}+3 H h\left(\partial_{i}, \omega\right) h\left(\partial_{j}, \omega\right)-H h(\omega, \omega) h_{i j} .
$$

Using the earlier computation relating $\Delta_{g} h$ (the tensorial Laplacian of $h$ ) and $\operatorname{tr}_{g} d^{2} h$, we obtain from this the evolution equation in terms of $L$ :

$$
L\left[h_{i j}\right]=-2\left[h_{i}^{k} \nabla_{\omega} h_{j k}+h_{j}^{k} \nabla_{\omega} h_{i k}\right]+\tilde{C}_{i j},
$$


where

$\tilde{C}_{i j}:=C_{i j}-2\left[h\left(\partial_{i}, \omega\right) h^{2}\left(\partial_{j}, \omega\right)+h^{2}\left(\partial_{i}, \omega\right) h\left(\partial_{j}, \omega\right)\right]-2\left(h^{3}\right)_{i j}-2\left(h^{2}\right)_{i j} h(\omega, \omega)-2 H h\left(\partial_{i}, \omega\right) h\left(\partial_{j}, \omega\right)$.

We may also write this purely in terms of the euclidean connection $d$ :

$$
L\left[h_{i j}\right]=-2\left[h_{i}^{k} d_{\omega} h_{j k}+h_{j}^{k} d_{\omega} h_{i k}\right]+\bar{C}_{i j},
$$

where

$$
\bar{C}_{i j}=C_{i j}+2\left[h\left(\partial_{i}, \omega\right) h^{2}\left(\partial_{j}, \omega\right)+h^{2}\left(\partial_{i}, \omega\right) h\left(\partial_{j}, \omega\right)\right]-2\left(h^{3}\right)_{i j}-2\left(h^{2}\right)_{i j} h(\omega, \omega)-2 H h\left(\partial_{i}, \omega\right) h\left(\partial_{j}, \omega\right) .
$$

Time derivatives and evolution equations for $\omega$ and $g$. The time derivative of $\omega$ is simply minus the time derivative of the $\mathbb{R}^{n}$ component of $N$. In addition, one computes easily that $(\nabla v) / v=S(\omega)$, where

$$
S(X):=S\left(X^{i} \partial_{i}\right)=h_{j}^{i} X^{j} \partial_{i}
$$

is the Weingarten operator. Hence

$$
\partial_{t} \omega=\nabla H+\frac{H}{v} \nabla v=\nabla H+H S(\omega) .
$$

For the metric and "inverse metric" tensors it follows from $\partial_{t} g_{i j}=\left(w_{i} w_{j}\right)_{t}$ and $w_{i t}=(v H)_{i}$ that

$$
\partial_{t} g_{i j}=v^{2}\left(H_{i} \omega^{j}+H_{j} \omega^{i}\right)+v^{2} H\left(h\left(\omega, \partial_{i}\right) \omega^{j}+h\left(\omega, \partial_{j}\right) \omega^{i}\right) .
$$

Then, using $\partial_{t} g^{i j}=-g^{i k} \partial_{t} g_{k l} g^{l j}$, we have

$$
\partial_{t} g^{i j}=-\left[(\nabla H)^{i} \omega^{j}+\left(\nabla H^{j}\right) \omega^{i}\right]-H\left[S(\omega)^{i} \omega^{j}+S(\omega)^{j} \omega^{i}\right] .
$$

Since we know the evolution equation of $\omega$, it is easy to obtain that of $g^{i j}$ :

$$
L\left[g^{i j}\right]=-L\left[\omega^{i} \omega^{j}\right]=-L\left[\omega^{i}\right] \omega^{j}+2 g^{k l}\left(\partial_{k} \omega^{i}\right)\left(\partial_{l} \omega^{j}\right)-\omega^{i} L\left[\omega^{j}\right] .
$$

Using $\partial_{k} \omega^{i}=h_{k}^{i}$, we find

$$
L\left[g^{i j}\right]=-2|h|_{g}^{2} \omega^{i} \omega^{j}+2\left(h^{2}\right)^{i j} .
$$

It is also easy to see that $\partial_{k} g^{i j}=-\left(h_{k}^{i} \omega^{j}+h_{k}^{j} \omega^{i}\right)$.

Evolution of the mean curvature. To compute the evolution equation for $H=g^{i j} h_{i j}$, we just need to remember that $g^{i j}$ is time-dependent:

$$
\left(\partial_{t}-\Delta_{g}\right) H=\left(\partial_{t} g^{i j}\right)\left(h_{i j}\right)+\operatorname{tr}_{g}\left[\left(\partial_{t}-\Delta_{g}\right) h\right]=-2 h(\nabla H, \omega)-2 H h^{2}(\omega, \omega)+\operatorname{tr}_{g}\left[\left(\partial_{t}-\Delta_{g}\right) h\right] .
$$

The result is

$$
\left(\partial_{t}-\Delta_{g}\right) H=H d_{\omega} H+|h|_{g}^{2} H+H h^{2}(\omega, \omega)-H^{2} h(\omega, \omega) .
$$

Since

$$
L[f]=\left(\partial_{t}-\Delta_{g}\right) f-H d_{\omega} f
$$

(for any $f$ ), we see that the equation in terms of $L$ has no first-order terms:

$$
L[H]=|h|_{g}^{2} H+H h^{2}(\omega, \omega)-H^{2} h(\omega, \omega) .
$$


One can also derive $L[H]$ from the expression $L\left[g^{i j} h_{i j}\right]=L\left[g^{i j}\right] h_{i j}+g^{i j} L\left[h_{i j}\right]-2 g^{k l}\left(\partial_{k} g^{i j}\right)\left(\partial_{l} h_{i j}\right)$.

Evolution of the Weingarten operator. The tensorial Laplacian of $S$ is the $(1,1)$ tensor $\Delta_{g} S$ with components $\Delta_{g} h_{j}^{k}$. We have

$$
\Delta_{g} h_{j}^{k}=g^{i k} \Delta_{g} h_{i j} \quad \text { or } \quad\left\langle\left(\Delta_{g} S\right) X, Y\right\rangle_{g}=\left(\Delta_{g} h\right)(X, Y) .
$$

The evolution equation is easily obtained:

$$
\begin{aligned}
& \left(\partial_{t}-\Delta_{g}\right) h_{j}^{k}=\left(\partial_{t} g^{i k}\right) h_{i j}+g^{i k}\left(\partial_{t}-\Delta_{g}\right) h_{i j} \\
& \quad=H \nabla_{\omega} h_{j}^{k}+H_{j} h_{l}^{k} \omega^{l}-H_{l} h_{j}^{l} \omega^{k}+|h|_{g}^{2} h_{j}^{k}+2 H S(\omega)^{k} h\left(\omega, \partial_{j}\right)-H h(\omega, \omega) h_{j}^{k}-H h\left(S(\omega), \partial_{j}\right) \omega^{k} .
\end{aligned}
$$

Remark. Since the components of $\nabla S$ are given by

$$
\left(\nabla_{\omega} S\right)\left(\partial_{j}\right)=\left(\nabla_{\omega} h_{j}^{k}\right) \partial_{k}, \quad \nabla_{\omega} h_{j}^{k}=d_{\omega}\left(h_{j}^{k}\right)+h^{2}\left(\omega, \partial_{j}\right) \omega^{k}-h\left(\omega, \partial_{j}\right) S(\omega)^{k},
$$

we see that upon setting $j=k$ and adding over $k$ we recover the evolution equation for $H$.

The evolution equation for $h_{j}^{k}$ in terms of $L$ follows from the calculation

$$
\begin{aligned}
L\left[h_{j}^{k}\right]= & L\left[g^{i k}\right] h_{i j}+g^{i k} L\left[h_{i j}\right]-2 g^{m n}\left(\partial_{m} g^{i k}\right)\left(\partial_{n} h_{i j}\right) \\
= & -2\left(\nabla_{\omega} h_{m}^{k}\right) h_{j}^{m}+\left(\partial_{j}|h|_{g}^{2}\right) \omega^{k} \\
& \quad+|h|_{g}^{2} h_{j}^{k}-H h(\omega, \omega) h_{j}^{k}+H S(\omega)^{k} h\left(\partial_{j}, \omega\right)+2 h^{3}\left(\partial_{j}, \omega\right) \omega^{k}-2\left(h^{2}\right)_{p}^{k} \omega^{p} h\left(\partial_{j}, \omega\right) .
\end{aligned}
$$

Setting $j=k$ and adding over $k$, we recover the earlier expression for $L[H]$.

Evolution of $|\boldsymbol{h}|_{\boldsymbol{g}}^{\mathbf{2}}$. That $g^{i j}$ is time-dependent introduces an additional term in the usual expression

$$
\left(\partial_{t}-\Delta_{g}\right)|h|_{g}^{2}=-2|\nabla h|_{g}^{2}+2\left\langle h,\left(\partial_{t}-\Delta_{g}\right) h\right\rangle_{g}+2\left(\partial_{t} g^{i j}\right)\left(h^{2}\right)_{i j} .
$$

Using the expressions given earlier, one easily finds

$$
\begin{aligned}
\left(\partial_{t}-\Delta_{g}\right)|h|_{g}^{2} & =-2|\nabla h|_{g}^{2}+H d_{\omega}|h|_{g}^{2}+2|h|_{g}^{4}-4 H h^{3}(\omega, \omega)-2 H|h|_{g}^{2} h(\omega, \omega), \\
L\left[|h|_{g}^{2}\right] & =-2|\nabla h|_{g}^{2}+2|h|_{g}^{4}-4 H h^{3}(\omega, \omega)-2 H|h|_{g}^{2} h(\omega, \omega) .
\end{aligned}
$$

\section{Acknowledgments}

It is a pleasure to thank Nicholas Alikakos for originally proposing to consider the problem of mean curvature flow for networks of surfaces meeting at constant angles and for his interest in this work. Most of the work on short-time existence was done during a stay at the Max-Planck Institute for Gravitational Physics in Golm (January-June, 2007). I am grateful to the Max-Planck Society for supporting the visit and to the Geometric Analysis group at the AEI for the invitation. Finally, thanks to Mariel Sáez for communicating the results of the Lens Seminar [Schnürer et al. 2007] and of her recent work on mean curvature flow of networks, partly in collaboration with Rafe Mazzeo [Mazzeo and Sáez 2007]. 


\section{References}

[Baconneau and Lunardi 2004] O. Baconneau and A. Lunardi, "Smooth solutions to a class of free boundary parabolic problems", Trans. Amer. Math. Soc. 356:3 (2004), 987-1005. MR 2004i:35317 Zbl 1092.35116

[Ecker 2004] K. Ecker, Regularity theory for mean curvature flow, Progress in Nonlinear Differential Equations and their Applications 57, Birkhäuser, Boston, MA, 2004. MR 2005b:53108 Zbl 1058.53054

[Ecker and Huisken 1991] K. Ecker and G. Huisken, "Interior estimates for hypersurfaces moving by mean curvature", Invent. Math. 105:3 (1991), 547-569. MR 92i:53010 Zbl 0707.53008

[Eidelman and Zhitarashu 1998] S. D. Eidelman and N. V. Zhitarashu, Parabolic boundary value problems, Operator Theory: Advances and Applications 101, Birkhäuser Verlag, Basel, 1998. MR 99h:35080 Zbl 0893.35001

[Guan 1996] B. Guan, "Mean curvature motion of nonparametric hypersurfaces with contact angle condition", pp. 47-56 in Elliptic and parabolic methods in geometry (Minneapolis, 1994), edited by B. Chow et al., A K Peters, Wellesley, MA, 1996. MR 98a:58045 Zbl 0870.35048

[Huisken 1984] G. Huisken, "Flow by mean curvature of convex surfaces into spheres", J. Differential Geom. 20:1 (1984), 237-266. MR 86j:53097 Zbl 0556.53001

[Huisken 1989] G. Huisken, "Nonparametric mean curvature evolution with boundary conditions", J. Differential Equations 77:2 (1989), 369-378. MR 90g:35050

[Lieberman 1996] G. M. Lieberman, Second order parabolic differential equations, World Scientific, River Edge, NJ, 1996. MR 98k:35003 Zbl 0884.35001

[Mantegazza et al. 2004] C. Mantegazza, M. Novaga, and V. M. Tortorelli, "Motion by curvature of planar networks", Ann. Sc. Norm. Super. Pisa Cl. Sci. (5) 3:2 (2004), 235-324. MR 2005d:53108 Zbl 1170.53313

[Mazzeo and Sáez 2007] R. Mazzeo and M. Sáez, "Self-similar expanding solutions of the planar network flow”, preprint, 2007. arXiv 0704.3113

[Protter and Weinberger 1984] M. H. Protter and H. F. Weinberger, Maximum principles in differential equations, Springer, New York, 1984. MR 86f:35034 Zbl 0549.35002

[Schnürer et al. 2007] O. Schnürer, A. Azouani, M. Georgi, J. Hell, N. Jangle, A. Koeller, T. Marxen, S. Ritthaler, M. Sáez, and F. S. B. Smith, "Evolution of convex lens-shaped networks under curve shortening flow", preprint, Lens Seminar, FU Berlin, 2007. arXiv 0711.1108

[Solonnikov 2003] V. A. Solonnikov, "Lectures on evolution free boundary problems: classical solutions", pp. 123-175 in Mathematical aspects of evolving interfaces (Funchal, 2000), edited by P. Colli and J. F. Rodrigues, Lecture Notes in Math. 1812, Springer, Berlin, 2003. MR 2011035 Zbl 1038.35063

[Stahl 1996] A. Stahl, "Convergence of solutions to the mean curvature flow with a Neumann boundary condition", Calc. Var. Partial Differential Equations 4:5 (1996), 421-441. MR 97f:58043 Zbl 0896.35059

[Struwe 1988] M. Struwe, "The existence of surfaces of constant mean curvature with free boundaries", Acta Math. 160:1-2 (1988), 19-64. MR 89a:53012 Zbl 0646.53005

Received 8 Dec 2008. Revised 8 Oct 2009. Accepted 17 Oct 2009.

ALEXANDRE FREIRE: freire@math.utk.edu

Department of Mathematics, University of Tennessee, Knoxville, TN 37996-1300, United States

http://www.math.utk.edu/ freire 


\title{
Analysis \& PDE
}

pjm.math.berkeley.edu/apde

EDITORS

\author{
EDITOR-IN-CHIEF \\ Maciej Zworski \\ University of California \\ Berkeley, USA
}

BOARD OF EDITORS

\begin{tabular}{|c|c|c|c|}
\hline Michael Aizenman & $\begin{array}{l}\text { Princeton University, USA } \\
\text { aizenman@math.princeton.edu }\end{array}$ & Nicolas Burq & $\begin{array}{l}\text { Université Paris-Sud 11, France } \\
\text { nicolas.burq@ math.u-psud.fr }\end{array}$ \\
\hline Luis A. Caffarelli & $\begin{array}{l}\text { University of Texas, USA } \\
\text { caffarel@math.utexas.edu }\end{array}$ & un-Yung Alice Chang & $\begin{array}{l}\text { Princeton University, USA } \\
\text { chang@math.princeton.edu }\end{array}$ \\
\hline Michael Christ & $\begin{array}{l}\text { University of California, Berkeley, USA } \\
\text { mchrist@math.berkeley.edu }\end{array}$ & Charles Fefferman & $\begin{array}{l}\text { Princeton University, USA } \\
\text { cf@math.princeton.edu }\end{array}$ \\
\hline Ursula Hamenstaedt & $\begin{array}{l}\text { Universität Bonn, Germany } \\
\text { ursula@math.uni-bonn.de }\end{array}$ & Nigel Higson & $\begin{array}{l}\text { Pennsylvania State Univesity, USA } \\
\text { higson@ math.psu.edu }\end{array}$ \\
\hline Vaughan Jones & $\begin{array}{l}\text { University of California, Berkeley, USA } \\
\text { vfr@math.berkeley.edu }\end{array}$ & Herbert Koch & $\begin{array}{l}\text { Universität Bonn, Germany } \\
\text { koch@math.uni-bonn.de }\end{array}$ \\
\hline Izabella Laba & $\begin{array}{l}\text { University of British Columbia, Canada } \\
\text { ilaba@math.ubc.ca }\end{array}$ & Gilles Lebeau & $\begin{array}{l}\text { Université de Nice Sophia Antipolis, France } \\
\text { lebeau@unice.fr }\end{array}$ \\
\hline László Lempert & $\begin{array}{l}\text { Purdue University, USA } \\
\text { lempert@math.purdue.edu }\end{array}$ & Richard B. Melrose & $\begin{array}{l}\text { Massachussets Institute of Technology, USA } \\
\text { rbm@math.mit.edu }\end{array}$ \\
\hline Frank Merle & $\begin{array}{l}\text { Université de Cergy-Pontoise, France } \\
\text { Frank.Merle@u-cergy.fr }\end{array}$ & William Minicozzi II & $\begin{array}{l}\text { Johns Hopkins University, USA } \\
\text { minicozz@ math.jhu.edu }\end{array}$ \\
\hline Werner Müller & $\begin{array}{l}\text { Universität Bonn, Germany } \\
\text { mueller@math.uni-bonn.de }\end{array}$ & Yuval Peres & $\begin{array}{l}\text { University of California, Berkeley, USA } \\
\text { peres@stat.berkeley.edu }\end{array}$ \\
\hline Gilles Pisier & $\begin{array}{l}\text { Texas A\&M University, and Paris } 6 \\
\text { pisier@math.tamu.edu }\end{array}$ & Tristan Rivière & $\begin{array}{l}\text { ETH, Switzerland } \\
\text { riviere@math.ethz.ch }\end{array}$ \\
\hline Igor Rodnianski & $\begin{array}{l}\text { Princeton University, USA } \\
\text { irod@math.princeton.edu }\end{array}$ & Wilhelm Schlag & $\begin{array}{l}\text { University of Chicago, USA } \\
\text { schlag@math.uchicago.edu }\end{array}$ \\
\hline Sylvia Serfaty & $\begin{array}{l}\text { New York University, USA } \\
\text { serfaty@ cims.nyu.edu }\end{array}$ & Yum-Tong Siu & $\begin{array}{l}\text { Harvard University, USA } \\
\text { siu@math.harvard.edu }\end{array}$ \\
\hline Terence Tao & $\begin{array}{l}\text { University of California, Los Angeles, USA } \\
\text { tao@math.ucla.edu }\end{array}$ & A Michael E. Taylor & $\begin{array}{l}\text { Univ. of North Carolina, Chapel Hill, USA } \\
\text { met@math.unc.edu }\end{array}$ \\
\hline Gunther Uhlmann & $\begin{array}{l}\text { University of Washington, USA } \\
\text { gunther@math.washington.edu }\end{array}$ & András Vasy & $\begin{array}{l}\text { Stanford University, USA } \\
\text { andras@math.stanford.edu }\end{array}$ \\
\hline Virgil Voiculescu & $\begin{array}{l}\text { University of California, Berkeley, USA } \\
\text { dvv@math.berkeley.edu }\end{array}$ & Steven Zelditch & $\begin{array}{l}\text { Johns Hopkins University, USA } \\
\text { szelditch@math.jhu.edu }\end{array}$ \\
\hline
\end{tabular}

\section{PRODUCTION}

apde@mathscipub.org

Silvio Levy, Scientific Editor

Sheila Newbery, Senior Production Editor

See inside back cover or pjm.math.berkeley.edu/apde for submission instructions.

The subscription price for 2010 is US \$120/year for the electronic version, and \$180/year for print and electronic. Subscriptions, requests for back issues from the last three years and changes of subscribers address should be sent to Mathematical Sciences Publishers, Department of Mathematics, University of California, Berkeley, CA 94720-3840, USA.

Analysis \& PDE, at Mathematical Sciences Publishers, Department of Mathematics, University of California, Berkeley, CA 94720-3840 is published continuously online. Periodical rate postage paid at Berkeley, CA 94704, and additional mailing offices.

APDE peer review and production are managed by EditFLOW ${ }^{\mathrm{TM}}$ from Mathematical Sciences Publishers.

PUBLISHED BY

mathematical sciences publishers

http://www.mathscipub.org

A NON-PROFIT CORPORATION

Typeset in IATE $_{\mathrm{E}} \mathrm{X}$

Copyright $(02010$ by Mathematical Sciences Publishers 


\section{ANALYSIS \& PDE}

\section{Volume $3 \quad$ No. $4 \quad 2010$}

Mean curvature motion of graphs with constant contact angle at a free boundary

ALEXANDRE FREIRE

Lifshitz tails for generalized alloy-type random Schrödinger operators

409

FRÉDÉRIC KLOPP and SHU NAKAMURA

Analytic continuation of the resolvent of the Laplacian and the dynamical zeta function

427

Vesselin PETKOV and LuCHEZAR StOYANOV 